\title{
7th Canadian Immunization Conference December 3 to 6, 2006 Winnipeg Convention Centre, Winnipeg, Manitoba
}

\section{ORAL ABSTRACTS Viral Diseases and Vaccines}

\section{O1}

POTENTIAL COST-EFFECTIVENESS OF VACCINATION
AGAINST HERPES ZOSTER AND POST-HERPETIC
NEURALGIA IN CANADA

M Brisson, Universite Laval; JM Pellissier, Merck Research Laboratories, Blue Bell, Pennsylvania, USA; C Quach, McGill University, Montreal, Quebec; P De Wals, Universite Laval, Quebec BACKGROUND: A live-attenuated varicella-zoster virus vaccine has been demonstrated in a large randomized, double-blinded, placebo-controlled clinical trial to prevent herpes zoster (HZ), post-herpetic neuralgia (PHN) and reduce the morbidity associated with incident $\mathrm{HZ}$ and its sequelae. The aim of the study is to assess the cost-effectiveness of vaccination against $\mathrm{HZ}$ and $\mathrm{PHN}$ in Canada.

METHODS: A decision analytic model was developed to estimate the burden of $\mathrm{HZ}$ and the cost-effectiveness of $\mathrm{HZ}$ vaccination in Canada. Canadian population-based data were used to estimate age-specific incidence, physician consultation, hospitalization and mortality rates. Quality Adjusted Life Years (QALYs) lost were calculated using QALY weight data available in the literature. Unit costs, taken from Canadian sources, were applied to the predicted HZ-associated health care resource use. The costeffectiveness of vaccination was estimated for various age cohorts (range: 50 to 80 years) and extensive sensitivity analyses were performed to assess uncertainty of results.

RESULTS: In Canada, the annual burden related to $\mathrm{HZ}$ is substantial, with an estimated 130,000 episodes of HZ, 20,000 cases of PHN and 30 deaths. Most of the pain and suffering (as measured by QALY loss) is born by adults over the age of 65 years and is due to PHN. Using $\$ 20,000$ per QALY gained as strong evidence for cost-effectiveness, $\mathrm{HZ}$ vaccination is cost-effective under a wide range of parameter assumptions and scenarios. Under base-case assumptions, the cost-effectiveness ratios for a cohort of vaccine recipients aged 65 years over a range of potential vaccine prices from $\$ 100$ to $\$ 200$ per dose were $\$ 11,000$ to $\$ 26,000$ per QALY gained. Results were most sensitive to the cost of PHN, duration of vaccine protection and the average QALY loss associated with HZ-related pain. CONCLUSION: Since both the health and economic burden of $\mathrm{HZ}$ and PHN are high, vaccination of the elderly is projected to be cost-effective.

\section{O2}

IMPACT OF A ONE DAY WORKSHOP ON PUBLIC HEALTH PROFESSIONALS' KNOWLEDGE, ATTITUDES AND BELIEFS ON HPV INFECTION, SCREENING AND VACCINATION V Gilca, Laval University Hospital Center, Quebec; C Sauvageau, Quebec Regional Public Health Department; F Lavoie, Laval University Hospital Center, Quebec; P Goggin, Quebec Institute of Public Health; M Steben, Quebec Institute of Public Health

BACKGROUND: Public health professionals (PHPs) play a key role in immunization programs. We designed a one-day training session on HPV for PHPs for all 18 regions of the province of Quebec.

OBJECTIVES: 1) To assess knowledge attitudes and beliefs on cervical cancer screening and HPV infection and vaccination; 2) To measure the change after a day-long workshop.
METHODS: For each region, the director of public health, the communicable diseases control coordinator, and one immunization staff member ( total $=54$ ) were invited to fill out a 99-item questionnaire sent by e-mail and anonymously faxed back. The same questionnaire was completed at the end of the workshop.

RESULTS: $63 \%$ PHPs responded pre- and $76 \%$ post-workshop: $65 \%$ vs $68 \%$ were women; $91 \%$ vs $94 \%$ had $>10$ years of professional experience. Knowledge, pre- vs post-workshop: $47 \%$ vs $87 \%$ accepted that HPV is a necessary cause for cervical cancer; $30 \%$ vs $85 \%$ that anogenital warts are not cancer precursors; $47 \%$ vs $98 \%$ that HPV 16 and 18 are responsible for more than $60 \%$ of cervical cancer cases (all $\mathrm{P}<0.05$ ). Beliefs pre- vs postworkshop: $37 \%$ vs $80 \%$ that vaccination would permit the commencement of screening later in life; $56 \%$ vs $88 \%$ that vaccination would reduce the frequency of screening interventions; $24 \%$ vs $95 \%$ respondents felt they received enough information on the HPV vaccines (all $\mathrm{P}<0.05$ ). Pre-workshop $>85 \%$ and post-workshop $>97 \%$ thought the HPV vaccines: should be administered to girls before the sexual debut; will be well accepted by the public and by vaccinators; and that a universal program would be useful.

CONCLUSION: HPV knowledge is insufficient in PHPs, but considerable improvements may be achieved after a relatively short workshop. Important modifications in PHPs beliefs may be achieved when evidencebased information is delivered. PHPs support universal HPV immunization of girls before sexual debut.

\section{O3}

\section{EPIDEMIOLOGY OF MUMPS IN CANADA}

T Lipskie, Immunization \& Respiratory Infections Division (IRID), Centre for Infectious Disease Prevention and Control (CIDPC), Public Health Agency of Canada (PHAC); A Medaglia, IRID, CIDPC, PHAC; J Macey, IRID, CIDPC, PHAC

BACKGROUND: By 1983, infant immunization with one dose of measles-mumps-rubella (MMR) vaccine was routine in Canada. In 1996/1997 every province and territory added a second dose of MMR to its routine schedule and one jurisdiction had used mumps-containing vaccine in their measles catch-up campaign. Recently, limited outbreaks in Nova Scotia and larger outbreaks in the United Kingdom and the United States prompted a closer investigation of disease burden in Canada.

METHODS: Descriptive statistics were used to explore the patterns of disease. Data were from the national Notifiable Disease Reporting System (NDRS), the 2004 National Immunization Coverage Survey, the Canadian Institute for Health Information and from Statistics Canada. RESULTS: The frequency and age distribution of mumps has changed. Since the introduction of immunization programs, the annual average has fallen to 88 cases. The largest proportion of cases were 10-14 year-olds in $1988-90$ but the majority of cases were over the age of 20 in 2003-05. In the past decade, there was an annual average of 25 hospitalizations with mumps as a primary diagnosis and one mumps death. Coverage estimates for a single dose of MMR vaccine by 2 years of age is $94 \%$ (close to the national goal of $97 \%$ ) although 2 doses of mumps vaccine by 7 years of age is only at $78 \%$. DISCUSSION: Mumps in Canada is affecting older age groups and there remain under-immunized cohorts based on age or opposition to vaccination. It remains to be seen if Canada will experience larger outbreaks in young adults or high risk settings like universities. Globally, outbreaks of mumps are attributed to under-vaccination, vaccine failure or waning immunity. As per World Health Organization recommendations, Canadian mumps surveillance at all levels should continue to monitor age 
groups at risk and immunization status. Vaccine failure and waning immunity may also warrant investigation.

\section{O4 \\ PARENTAL KNOWLEDGE AND BELIEFS SURROUNDING VARICELLA AND THE VARICELLA VACCINE}

M MacLellan, St Francis Xavier University

INTRODUCTION: In January, 2003, a publically funded varicella program was put in place in Nova Scotia. The objectives of this study were: to determine the number of parents surveyed who had their child (ren) immunized against varicella, to explore parental beliefs regarding reasons for and against immunization, to establish parental views regarding the seriousness of the varicella and to determine who the health care providers were and their preference for provider of the vaccine.

METHOD: A quantitative study exploring parent's knowledge and beliefs around varicella and the varicella vaccine was conducted in the northeastern area of Nova Scotia in June of 2005. A questionnaire was distributed to a convenient sample of 148 parents who had children between the ages of $1-5$ years.

RESULTS: Over $60 \%$ did not have all of their children immunized against varicella. The main reasons for not immunizing ranged from their child (ren) already had varicella (46\%), it wasn't publically funded (16\%) and their doctor did not recommend the vaccine $(10 \%)$. The major reasons given for immunizing included they did not want their child to be sick $(55 \%)$, a recommendation from a health care provider $(15 \%)$, and they did not want their child to infect another child (13\%). Fifty six percent did not view varicella infection as a serious disease. There was statistical significance between knowing someone who became very ill with varicella and their perception of the seriousness of the disease. The majority in the study $(84 \%)$ received their vaccine from their family physician with (67\%) preferring to receive the vaccine from their doctor.

CONCLUSIONS: There are many variables that affect parental decisions regarding the varicella vaccine. Health care providers and especially nurses who work with parents of young children can play a major role in addressing those variables.

\section{O5}

UPDATE ON MOLECULAR EPIDEMIOLOGY OF MEASLES MUMPS, RUBELLA AND VARICELLA-ZOSTER IN CANADA

T EisBrenner, National Microbiology Laboratory; GA Tipples, National Microbiology Laboratory, Public Health Agency of Canada (PHAC); University of Manitoba; J Ball, National Microbiology Laboratory, PHAC; J Hiebert, National Microbiology Laboratory, PHAC; GA Peters, National Microbiology Laboratory, PHAC; SD Tyler, National Microbiology Laboratory, PHAC; JF Macey, Centre for Infectious Disease Prevention and Control, PHAC OBJECTIVES: We describe the current status of measles, mumps, rubella and varicella-zoster virus (VZV) molecular epidemiology activities in Canada. With the recent addition of varicella vaccine, all four viruses are included in publicly funded vaccine programs across Canada. Surveillance is needed to monitor changing epidemiology and impact on disease burden. Molecular epidemiology supports surveillance by differentiating between wild-type and vaccine strains, tracing transmission pathways and importations, documenting endemic strain elimination, and determining the probable epidemiology of sporadic cases.

METHODS: The WHO has developed genotyping standards for measles and rubella. A standardized mumps genotyping system has also been published. Genotyping involves PCR-based amplification, DNA sequencing and phylogenetic comparison with reference strains. VZV genotyping involves identifying strain-specific single-nucleotide polymorphisms.

RESULTS: Since 2001, various measles genotypes have been identified in Canada (A, B2, B3, D3, D4, D6, D5, D8, D9, H1) and subsequently linked with importations from various regions (USA, Philippines, Pakistan, Germany, Mexico, New Zealand, Bangladesh, Korea). Comparatively few rubella and mumps specimens have been collected for genotyping over this time period, however with notable identifications of a new $1 \mathrm{~g}$ strain of rubella associated with a 2005 Ontario rubella outbreak (Netherlands linked) and a mumps G strain associated with UK/France and USA travel in 2006. Four distinct VZV genotype clades have been identified and associated with characteristic geographic distributions.

CONCLUSIONS: The variety of import-related measles genotypes detected in Canada, in the absence of sustained transmission in the general population, is consistent with interruption of endemic transmission and maintained indigenous measles elimination. Continued specimen collection for molecular epidemiology is necessary to document measles elimination. Increased specimen submission for rubella and mumps genotyping is needed to provide molecular epidemiology evidence for elimination/reduction of these vaccine preventable diseases. VZV genotyping methods are currently available in Canada which allow differentiation between intra- and interstrain variation.

\section{O6 DETECTION OF HUMAN INFLUENZA VIRUS IN THE STOOL OF CHILDREN}

$S$ Wootton, Vaccine Evaluation Center; A Mak, BC Centre for Disease Control (CDC); M Petric, BC CDC; T Kwindt, BC CDC; D Scheifele, UBC; D Skowronski, BC CDC

BACKGROUND: Influenza infection is common among children. Most experience typical respiratory symptoms but some, especially children age $<6$-mos, also develop gastrointestinal (GI) symptoms. Avian influenza H5N1 has been detected by reverse-transcriptase polymerase chain reaction (RT-PCR) in the GI tract of affected children but this has not been reported in association with human influenza viruses. We explored detection of human influenza virus in the stool of very young children hospitalized with influenza.

METHODS: Up to three stool samples were collected daily from children age <24-mos admitted to BC Children's Hospital (BCCH) with laboratoryconfirmed influenza from January-May, 2006. Laboratory-confirmation was by immunofluorescence testing on nasopharyngeal wash (NPW) specimens, performed at $\mathrm{BCCH}$ Microbiology Laboratory. Stool specimens were refrigerated at $4^{\circ} \mathrm{C}$ and transported to the BC Centre for Disease Control Virology Laboratory for RT-PCR examination for influenza.

RESULTS: 4 (80\%) of 5 enrolled children submitted stool samples; 2 children submitted 3 specimens and 2 submitted 1 specimen. Influenza virus was detected in stool specimens of 2 children: a 9-mo-old vaccinated female (with Type B virus) with sickle cell disease who presented with diarrhea and a 5-week old healthy female who did not have diarrhea (Type A virus). Results of NPW screens for both children matched the influenza type detected in their stool. The mean time between onset of symptoms and stool collection was 2.7 days for children with influenza-positive stools and 9.5 days for children with influenza-negative stools.

CONCLUSION: Despite small sample size, influenza virus, both types A and B, was identified in the stool of half of the children tested. Influenza may be particularly evident in stool early in the course of illness. The assumption that detection of human influenza virus is generally confined to respiratory specimens should be revisited. GI shedding should be further evaluated.

\section{O7}

\section{THE HEALTH AND ECONOMIC BURDEN OF HPV INFECTION, GENITAL WARTS, CERVICAL DYSPLASIA AND CERVICAL CANCER IN CANADA}

M Brisson, Universite Laval; N Van De Velde, Universite Laval, Quebec; MC Boily, Imperial College, London, United Kingdom; P De Wals, Universite Laval, Quebec

BACKGROUND: Clinical trials have shown prophylactic HPV vaccines to be effective against persistent HPV infection, cervical intraepithelial neoplasia (CIN) and genital warts. To estimate the potential impact of HPV vaccination at a population level it is necessary to have a good understanding of the burden of the disease. Although epidemiological and economic data on HPV and its related diseases exist for Canada, they are sparse and incomplete. Models provide a formal framework to synthesize information from various sources. The aim of this study was to estimate the health and economic burden of HPV in Canada, using a mathematical model. 
METHODS: A cohort model of the natural history of HPV infection and disease was developed. Model simulations where based on multiple parameter sets that fit Canadian age and type-specific data for infection, CIN, cervical cancer and genital warts. Health care resource use and related costs were applied to modeled HPV-associated outcomes. Probability sensitivity analysis was performed to capture the uncertainty surrounding the economic parameters.

RESULTS: Our model predicts that, each year in Canada, 3 million women are infected with HPV, which results in 47,000 episodes of genital warts, 203,000 naturally occurring CIN, 1,200 cervical cancer cases and 500 cervical cancer deaths. The treatment and diagnosis of CIN, cervical cancer and genital warts are predicted to cost the health care system between $\$ 273-\$ 358$ million each year.

CONCLUSION: The burden of HPV-related disease is substantial in Canada. There is therefore a significant potential for reducing it through HPV vaccination. More empirical studies are needed to capture the full burden of HPV. Areas of further research are the psychosocial impact and the indirect costs of CIN, cervical cancer, genital warts and the burden of other HPV related disease such as vulva, vaginal, anus, penile, and head and neck cancers.

\section{ORAL ABSTRACTS Public Health Research}

\section{O8 \\ REPORTED CASES OF FACIAL PARALYSIS FOLLOWING IMMUNIZATION IN CANADA}

H Anyoti, Public Health Agency of Canada; B Law, Public Health Agency of Canada

INTRODUCTION: Facial paralysis is a rare adverse event and has been reported post-surveillance as an event temporally associated with influenza vaccine.

METHOD: Case reports of facial paralysis following administration of vaccines approved for use in Canada from Jan 1997 to Jun 2006 were extracted from Canadian Adverse Events Following Immunization Surveillance System (CAEFISS). All were reviewed individually and all adverse events were classified using WHO-ART codes.

RESULTS: A total of 59 case reports of facial paralysis were received by Public Health Agency of Canada (PHAC). The male to female ratio was 1:2.4. The age-range of reported cases was 1 month to 78 years with the greatest number of reports for the age-groups of $18 \leq 65$ years $55.9 \%(n=33)$ and $7 \leq 13$ years $22.0 \%(n=13)$. A specific diagnosis of Bell's palsy was made in $39.6 \%(n=36)$ of the cases. Medical attention was indicated for $83.1 \%$ $(n=49)$ of the cases of which, $10.2 \%(n=6)$ were hospitalized, $15.3 \%(n=9)$ were seen in the emergency department, $57.6 \%(n=34)$ were seen as outpatients and $17.0 \%(n=10)$ sought no medical attention.

Outcome data was specified for $64.4 \%(n=38)$ of which $39.0 \%(n=23)$ had fully recovered, $13.6 \%(n=8)$ were recovering and $11.9 \%(n=7)$ had residual effects at the time of reporting. For $48(81.4 \%)$ of reports only a single vaccine was given and these included in order of frequency, influenza (24 reports), hepatitis B (12), Td toxoids (5), meningococcal conjugate C (4) and single reports for varicella, rabies and meningococcal AC polysaccharide. Of the $28(47.5 \%)$ cases selected for review by the Advisory Committee on Causality Assessment 20.3\% ( $n=12$ ) could not be reviewed due to insufficient data, $3.4 \%(\mathrm{n}=2)$ were found to be unclassifiable, $8 \%$ $(\mathrm{n}=4)$ possibly related, $1.7 \%(\mathrm{n}=1)$ probably related and $15.1 \%(\mathrm{n}=9)$ unlikely/unrelated to immunization.

CONCLUSION: Facial paralysis, including Bell's palsy, is rarely reported following immunization in Canada. Most cases that can be reviewed by an expert panel are judged as not likely related to immunization and the outcome is good for the majority of reported cases.

\section{O9}

\section{COST-EFFECTIVENESS OF UNIVERSAL HEPATITIS A VACCINATION IN CANADA}

C Bauch, University of Guelph; A Anonychuk, GlaxoSmithKline, University Health Network; BZ Pham, GlaxoSmithKline, University Health Network, University of Toronto; V Gilca, Universite Laval, INSPQ; B Duval, Universite Laval, INSPQ; MK Krahn, University of Toronto, University Health Network

INTRODUCTION: The incidence of Hepatitis A (HA) has declined in most developed nations over the past century while remaining high in many other countries. Given a relatively low disease burden in Canada on the one hand and a safe and effective vaccine on the other, it is not clear whether universal vaccination should be implemented. The purpose of this study is to evaluate the cost-effectiveness of universal HA vaccination in Canada.

METHODS: The use of dynamic models in cost-effectiveness analyses can, in principle, make them more accurate. For the present study, an agestructured dynamic model was developed and parameterized using (1) clinical data on the course of HA infection, (2) a systematic review of HA seroprevalence studies in Canada, (3) catalytic modeling to estimate the true infection rate of HA in Canada by adjusting for subclinical infection and underreporting, and (4) survey data on infection rates due to travel in HA endemic countries. Costs were obtained from a systematic literature review and Ontario and Quebec administrative data. Vaccination costs, direct infection costs, and time costs were also estimated.

RESULTS: Addition of a universal HA vaccination to the existing school-based HB program at the age of 9 via combined vaccine provides $\$ 52,000$ per QALY for the payer perspective and $\$ 30,000$ per QALY for the societal perspective, relative to no vaccination. One HA-only dose at age 4 in clinic followed by one combined HA/HB dose at age 9 in school provides ratios of $\$ 26,000$ and $\$ 32,000$ per QALY for payer and societal perspectives respectively.

CONCLUSION: Universal vaccination appears to be economically attractive, relative to no vaccination. Definitive policy recommendations must wait until universal vaccination can be compared to the current targeted approach which appears to have been effective in reducing HAV disease burden.

\section{O10 \\ VERSATILITY OF ELECTRONIC IMMUNIZATION DATABASE FOR DETERMINING COVERAGE RATES}

J Chomyn, Public Health Services

INTRODUCTION: Regional immunization coverage rates are not necessarily a good indicator of disease risk. Pockets of poor coverage could result in a critical mass of susceptible individuals and open the door to a communicable disease outbreak. The utilization of the Saskatchewan Immunization Management System (SIMS), a web-based electronic database, has enabled us to determine age appropriate coverage rates by antigen at a community and neighbourhood level.

METHOD: SIMS was used to identify regional level coverage rates for 2 - and 7-year-olds. Postal codes then identified coverage rates at neighbourhood levels. This revealed an overall 2-year-old coverage rate of $73 \%$. However, range of coverage between neighbourhoods varied from $47 \%$ to 93\%. A study done to examine measles, mumps and rubella immunization uptake identified that neighbourhood characteristics were good predictors of coverage rates. An immunization outreach nurse worked with the low coverage communities to identify barriers to immunization and develop strategies to minimize these barriers.

RESULTS: Information gleaned was used to set neighbourhood and community-wide immunization coverage rate targets. Staff had to rethink delivery of services and consider re-prioritizing resources toward areas of greater need. Strategies were implemented to address disparities in immunization coverage balancing the affected community's suggestions with best practice literature information.

CONCLUSIONS: Electronic immunization databases are invaluable tools in determining coverage rates and planning interventions at a subregional level. Low immunization rates are often a symptom of a greater 
problem in health disparity. Our research has shown that areas with the lowest immunization rates also have significant variations in underlying determinants of health. These include education, income levels, teenage pregnancy, suicide, sexually transmitted disease and mortality rates. Decreasing neighbourhood disparities in immunization should be part of a larger initiative to reduce health disparities and minimize the risk of vaccine preventable disease outbreaks at the same time.

\section{O11}

HEALTH PROFESSIONALS SURVEY: KNOWLEDGE, ATTITUDES, AND PRACTICES ABOUT HPV VACCINES USE AND THEIR POTENTIAL IMPACT ON CERVICAL CANCER SCREENING

V Gilca, Laval University Hospital Center; S Dobson, British

Columbia University; I Gemmill, Kingston, Frontenac and Lennox \& Addington Public Health Unit; S McNeil, Dalhousie University; C Sauvageau, Quebec Regional Public Health Unit; F Lavoie, Laval University Hospital Center

BACKGROUND: The implementation of new HPV vaccines is a challenging issue because it is the first vaccine against a sexually transmitted disease, addressed to women only, expected to prevent a cancer, and to be given in a synergy with an existing screening program. In 2006, we conducted a survey in Canadian obstetrics-gynecologists (OGs), pediatricians, general practitioners, and public health professionals (PHPs).

OBJECTIVES: To measure knowledge, attitudes and practices regarding HPV-related diseases and their prevention; to provide information regarding physicians' opinions on HPV vaccine use and its impact on screening interventions.

METHODS: The survey was conducted in four Canadian provinces (Nova Scotia, Quebec, Ontario and British Columbia). A self-administered questionnaire was mailed to 1500 randomly selected physicians. The Systems Model of Clinical Preventive Care was used as theoretical base for questionnaire building (Walsh and McPhee). The survey was conducted by following procedures described by Dillman.

RESULTS: Here we present preliminary results obtained in Quebec OGs and PHPs. The majority of OGs (92\%) and PHPs (91\%) think the HPV vaccine should be given to girls before sexual debut; respectively $30 \%$ and $38 \%$ that an immunization program will permit the beginning of screening later in life; $68 \%$ and $56 \%$ that it will reduce the frequency of screening interventions; $79 \%$ and $75 \%$ that it will reduce the number of post-screening follow-up interventions; $85 \%$ and $91 \%$ will recommend the HPV vaccine to their patients if it is publicly funded, but only $78 \%$ and $61 \%$ if patients have to pay for the vaccine.

DISCUSSION: HPV vaccine is viewed as an important tool in cervical cancer prevention and almost all responders think it should be given to girls before sexual debut. Physicians expect important changes in screening of immunized women. The vaccine recommendation by physicians might be influenced by the need to pay for the vaccine.

\section{2}

\section{THE EFFECT OF A UNIVERSAL INFLUENZA} IMMUNIZATION PROGRAM ON INFLUENZA-RELATED HOSPITALIZATIONS AND MORTALITY

J Kwong, Institute for Clinical Evaluative Sciences; DG Manuel, Institute for Clinical Evaluative Sciences; TA Stukel, Institute for Clinical Evaluative Sciences; J Lim, Institute for Clinical Evaluative Sciences; AJ McGeer, Mount Sinai Hospital, Toronto, Ontario; R Upshur, University of Toronto Joint Centre for Bioethics; H Johansen, Statistics Canada; C Sambell, Statistics Canada; WW Thompson, Center for Disease Control and Prevention INTRODUCTION: In October 2000, Ontario initiated the world's first large-scale universal influenza immunization program (UIIP) to provide free influenza vaccines for the entire population aged 6 months or older. We evaluated the effect of Ontario's UIIP on hospitalizations and mortality compared to targeted influenza immunization programs (TIIP) in other provinces.

METHODS: Weekly hospitalizations for influenza-related conditions (pneumonia and influenza, acute respiratory diseases, and chronic obstructive pulmonary disease) and respiratory and circulatory $(R \& C)$ conditions (1993-2004), and deaths among seniors from $R \& C$ conditions and all causes (1993-2003) for the 10 Canadian provinces were extracted from national hospital discharge abstract and vital statistics databases. We compared relative outcome rates before and after UIIP using Poisson GEE regression models, controlling for age, sex, region, influenza season covariates, and temporal trends.

RESULTS: After UIIP introduction, hospitalizations for the overall population decreased similarly in Ontario and other provinces $(\mathrm{RR}=0.93$ vs. $0.94, \mathrm{p}=0.15)$ for influenza-related conditions, but more in Ontario than other provinces for $\mathrm{R} \& \mathrm{C}$ conditions ( 0.96 vs. 1.00 , $\mathrm{p}<0.001)$. Consistent with age-specific vaccination rate changes, influenza-related hospitalizations decreased more in Ontario than other provinces for those aged 20-49, but the opposite was seen for those aged 75 years or older. Restricting the analysis to seasons with good vaccine match resulted in greater UIIP effects for influenza-related hospitalizations ( 0.91 vs. $0.93, p=0.01$ ). Deaths from $R \& C$ conditions among seniors decreased less in Ontario than in other provinces (0.95 vs. 0.91, $\mathrm{p}=0.02$ ), consistent with smaller increases in vaccination rates over time in the elderly. There was no difference between Ontario and other provinces in all-cause mortality. Additional analyses suggested consistency, specificity and the presence of a biological gradient in the association between influenza vaccination and outcomes.

CONCLUSIONS: Introduction of Ontario's UIIP in 2000 was associated with decreases in outcomes that were dependent on vaccine uptake and a good vaccine match.

\section{3}

\section{A CANADIAN SURVEY OF IMMUNIZATION PRACTICES FOLLOWING HEMATOPOIETIC STEM CELL TRANSPLANTATION IN ADULTS}

\section{MacFarlane Conrad, Canadian Centre for Vaccinology;} S Couban, QEII Health Sciences Centre, Halifax, Nova Scotia; S McNeil, Canadian Centre for Vaccinology, Halifax, Nova Scotia BACKGROUND: Hematopoietic stem cell transplantation (HSCT) has become an effective therapeutic and often curative treatment for a variety of malignant and non-malignant conditions. The conditioning of patients prior to HSCT replacement ablates immune memory, rendering patients vulnerable to a variety of vaccine-preventable diseases. To reduce the risk of infection, adult HSCT patients are re-immunized following transplantation. However, no standardized Canadian guidelines exist and existing Canadian recommendations regarding necessary vaccines and schedules post-HSCT allow for considerable variation in implementation. We surveyed adult HSCT centres in Canada regarding post-HSCT immunization protocols in order to evaluate degree of variability and consistency with Canadian recommendations.

METHODS: All 10 Canadian centres offering adult HSCT were contacted and asked to submit their post-HSCT vaccination protocols, both for allogeneic and autologous transplant patients. The vaccines used, their dosage and their timing were compared to determine the extent of variation.

RESULTS: Nine of ten centres performing HSCT in Canada responded (90\%). Seven centres (77.7\%) offer the same immunizations to all HSCT recipients; two centres have differing vaccination schedules for autologous and allogeneic transplant recipients. Haemophilus influenzae type B and pneumococcal vaccines are provided in $100 \%$ of centres regardless of mode of HSCT. Diphtheria, tetanus, measles, mumps, and rubella vaccines are provided by all centres for allogeneic patients and by $90 \%$ of centres for autologous patients; poliovirus and influenza vaccines are provided in $77.8 \%$ of centres. Hepatitis A, Hepatitis B, meningococcus, and varicella vaccines are offered sporadically, usually among patients deemed to be "at risk". The number and timing of vaccine doses varies widely from institution to institution.

CONCLUSIONS: Despite recommendations for certain vaccines, there is considerable variation between centres regarding which infections are felt to pose the greatest risk to HSCT patients and thus, which vaccinations should be implemented. Additionally, the timing and number of doses is quite different both within and between provinces. A nation-wide 
consensus is necessary to ensure that all Canadian HSCT recipients are adequately protected against these preventable infections.

\section{O14 \\ INFLUENZA AND THE HEALTH CARE WORKFORCE: IDENTIFYING BARRIERS AND FACILITATORS TO VACCINE UPTAKE IN BRITISH COLUMBIA}

C Masaro, BC Centre for Disease Control; DM Skowronski, University of British Columbia and BC Centre for Disease Control; JA Buxton, University of British Columbia and BC Centre for Disease Control; K Lockhart, University of British Columbia; A Yassi, University of BC and Occupational Health and Safety Agency INTRODUCTION: Vaccinating health care workers (HCWs) reduces their personal risk of influenza and the probability of transmitting influenza to patients and family members. Annual immunization rates among HCWs remain low despite promotional campaigns and provincial and workplace policies focusing on immunization as a duty of care. Few studies have explored the influence of workplace systems, culture, and environmental factors on HCW immunization decision-making. The objective of this qualitative study was to explore the contribution of both individual-and system-level workplace factors influencing $\mathrm{HCW}$ influenza immunization. METHODS: A semi-structured interview guide was developed to explore determinants and deterrents of influenza immunization. HCWs were selected from health care facilities across BC. Facilities were chosen by type (acute/long-term care), size (large/small), location (urban/rural), and historic immunization coverage (high/low vaccine uptake). Program managers were also interviewed. Data were collected through 20 to 30 minute telephone interviews.

RESULTS: $103 \mathrm{HCW}$ from 12 facilities were recruited, including $79 \mathrm{vac}-$ cinated and 24 non-vaccinated staff. Eleven of 12 program managers were interviewed. The dominant theme emerging was that the decision to be immunized is primarily influenced by: 1) perceived personal health risk or benefit (self or family), 2) workplace policy as it overlaps with personal considerations (ability to work and be paid) and, 3) access to in-depth education, preferably one-on-one by an independent and knowledgeable expert. Availability of vaccine clinics and other workplace factors were less prominent, in contrast to other studies.

CONCLUSIONS: Further research is needed to explore in greater depth the dominant themes that emerged in this study. Specific strategies incorporating HCW perspectives should be developed with individual-level education and personal benefits emphasized over mass promotional and patient-centred campaigns. Direct benefits to HCWs and their families need to be highlighted. Local expert champions and trusted opinion leaders may best address ongoing $\mathrm{HCW}$ questions and concerns.

\section{O15}

ISSUES IN UNDER-IMMUNIZED POPULATIONS: THE TALE OF THREE OUTBREAKS

B Warshawsky, Middlesex-London Health Unit; M Metcalfe, Oxford County Board of Health, Woodstock, Ontario; S Wilson-Clark, previously with Oxford County Board of Health, Woodstock, Ontario; D Sider, Regional Niagara Public Health Unit, St Catherines, Ontario; M Bragg, Oxford County Board of Health, Woodstock, Ontario; V Dubey, previously with University of Toronto, Toronto, Ontario; MA Simpson, Middlesex-London Health Unit, London, Ontario; C Egan, previously with Middlesex-London Health Unit, London, Ontario; A Locker, Middlesex-London Health Unit, London, Ontario; G Pollett, Middlesex-London Health Unit, London, Ontario

Oxford County and Middlesex-London, two adjacent communities in southwestern Ontario, have two large under-immunized communities in close proximity - a Netherlands Reform Community of approximately 4000 people with close connections to the Netherlands, and an Amish population of approximately 700 people, with close connections to other Amish communities in Ontario and the United States. This presentation will review three outbreaks involving these under-immunized populations, and strategies to prevent and manage outbreaks in these communities.
Outbreak 1: A large polio outbreak in 1978 in the Netherlands spread to Netherlands Reform communities in Canada resulting in one paralytic case in Alberta, two paralytic cases in British Columbia and six paralytic cases in Ontario. From Ontario, subsequent transmission to the Amish population resulted in 15 cases of polio disease in the United States and two additional cases in Ontario.

Outbreak 2: A large rubella outbreak in the Netherlands in 2004-2005 resulted in laboratory-confirmed rubella in 387 people, including 29 pregnant women. At least three newborns were found to have multiple serious birth defects. In April 2005, spread to Ontario resulted in 311 confirmed cases of rubella, mostly in Oxford County, including 10 pregnant women. To date, no newborns have been identified with congenital rubella syndrome in Ontario.

Outbreak 3: In fall 2005, five children in an Amish community in Minnesota were identified as asymptomatically excreting polio-virus in their stool. Because of social connections with the Amish community in Middlesex-London, enhanced surveillance and infection control measures were initiated. No evidence of transmission was found.

Strategies to prevent and manage outbreaks of vaccine preventable disease in under-immunized communities include: establishing pre-existing relationships with open, frequent communication; respect for underlying religious and cultural beliefs; convenient easily accessible immunization opportunities; early notification of world-wide outbreaks to local health care providers; and use of control measures other than immunization.

\section{ORAL PRESENTATIONS Bacterial Diseases and Vaccines}

\section{6}

IMPACT OF INFANT VACCINATION AGAINST INVASIVE
PNEUMOCOCCAL DISEASE IN VANCOUVER-COASTAL
AND FRASER HEALTH AUTHORITIES
AND FRASER HEALTH AUTHORITIES

G Bjornson, Vaccine Evaluation Center; D Scheifele, Vaccine Evaluation Center, Vancouver, British Columbia; J Bettinger, Vaccine Evaluation Center, Vancouver, British Columbia; M Mozel, Vaccine Evaluation Center, Vancouver, British Columbia; P Daly, Vancouver-Coastal Health Authority, Vancouver, British Columbia; L Gustafson, Fraser Health Authority, Surrey, British Columbia; R Guasparini, Fraser Health Authority, Surrey, British Columbia; D Patrick, BC Center for Disease Control, Vancouver, British Columbia; G Tyrrell, National Streptococcus Laboratory, Edmonton, Alberta

OBJECTIVE: The purpose of this study was to assess the effectiveness of the new, routine infant immunization program against invasive pneumococcal infection implemented in July, 2003, in British Columbia using Prevnar ${ }^{\circledR}$ vaccine.

METHODS: Active surveillance for invasive pneumococcal infection in children $<16$ years of age was conducted at all Vancouver-Coastal and Fraser Health Authority hospitals and laboratories from September 2001 to December 2005. Invasive disease was defined as isolation of S pneumoniae from a normally sterile body site.

RESULTS: 276 cases were identified: 186 in the 2 years prior to program implementation and 90 in the 28 months post-inception. Incidence rate in children 6-23 months of age fell from 125.6/100,000 (95\% CI 101.7, 153.6) to $39.4 / 100,000(95 \%$ CI $26.6,56.3)$ in the first 2 years of the routine immunization program. Of the 38 cases that occurred in children less than 28 months of age after program introduction, only 9 were age-eligible for the routine vaccine program which did not include a catch-up component: 8 of these 9 cases were non-preventable, involving non-vaccine serotypes. One of the 9 cases was potentially preventable (involving serotype 6B) however the child had no record of pneumococcal vaccination.

CONCLUSIONS: The introduction of Prevnar ${ }^{\circledR}$ vaccine in infancy resulted in a 3.2 fold decrease in the rate of invasive pneumococcal disease in children 6-23 months of age within 28 months of program introduction. 


\section{7}

THE INTERNATIONAL CIRCUMPOLAR SURVEILLANCE SYSTEM FOR POPULATION-BASED SURVEILLANCE OF INVASIVE PNEUMOCOCCAL DISEASE 1999-2004

M Bruce, CDC; T Cottle, Arctic Investigations Program, CDC, Anchorage, Alaska; S Deeks, Centre for Infectious Disease Prevention and Control; Public Health; M Lovgren, National Centre for Streptococcus, Edmonton, Alberta; L Jette, Quebec Public Health Laboratory, Quebec; T Hennessy, Arctic Investigations Program, CDC, Anchorage, Alaska; D Parks, Arctic Investigations Program, CDC, Anchorage, Alaska; K Kristinsson, Deparment of Clinical Microbiology, Landspitali Hospital, Reykjavik; K Brinklov Jensen, Institution of the Chief Medical Officer, Nuuk, Greenland; O Lovoll, Norwegian Institute of Public Health, Oslo, Norway; P Nuorti, National Public Health Institute, Helsinki \& Oulu, Finland; A Nystedt, Department of Infectious Diseases, Sunderby Hospital, Lulea, Sweden; E Herva, National Public Health Institute, Helsinki \& Oulu, Finland; A Koch, Statens Serum Institut, Copenhagen, Denmark; A Parkinson, CDC, Anchorage, Alaska BACKGROUND: The International Circumpolar Surveillance (ICS) Project is a population-based surveillance network for invasive bacterial disease in the US Arctic, Alaska (AK), Northern Canada (N Can), Greenland (GN), Iceland (IC), Norway (Nor), Northern Sweden (N Sweden) and Finland (Fin). Among circumpolar countries, the 7-valent conjugate vaccine (pcv7) has been used for routine infant immunization in AK since 2001 and in selected areas in N Can since 2002.

METHODS: We defined a case of invasive pneumococcal disease (IPD) as illness in a surveillance area resident with isolation of Streptococcus pneumoniae from a normally-sterile site. We analyzed data on IPD from AK and N Can (Jan 1999-Dec 2004), and from GN, IC, Nor, Fin (Jan 2000. Dec 2004) and N Sweden (2003-2004) to determine: 1) common clinical syndromes, 2) disease rates by country, 3) serotype distribution and 4) antimicrobial susceptibility patterns.

RESULTS: A total of 9,251 cases of laboratory-confirmed IPD were reported from AK (647), N Can (226), GN (51), IC (236), Nor (4,712), $\mathrm{N}$ Sweden $(65)$ and Fin $(3,314)$. Case-fatality ratios varied from 5.0 to $27.0 \%$. Pneumonia (46\%), septicemia (26\%), and meningitis (8\%) were the most common clinical presentations. Annualized rates of IPD in aboriginals in $\mathrm{AK}$ and $\mathrm{N}$ Can were 43 and 38 cases per 100,000 persons, respectively. Rates of IPD in children $<2$ years of age and persons $>2$ years of age ranged from $21-137$ and $9-24$ cases per 100,000 persons, respectively. In AK, the rate of IPD in children < 2 with pcv7 serotypes declined by $>85 \%$ after routine vaccination; from 137 in 1999-2000 to 18 in 2001-2004 $(\mathrm{p}<0.001)$. The proportion of isolates fully-resistant to penicillin varied from $<1 \%$ in Fin to $5.6 \%$ in AK.

CONCLUSIONS: Rates of IPD are high in aboriginals and children $<2$ years of age residing in Arctic countries. After introduction of pcv 7 in $\mathrm{AK}$, rates of disease in children $<2$ years of age with pcv 7 serotypes rapidly declined. Continued surveillance is needed to determine the impact of pcv7 in AK and areas of N Can. High IPD rates in children $<2$ warrant consideration of pcv7 use in other circumpolar countries.

\section{O18}

INCREASE IN INVASIVE PNEUMOCOCCAL DISEASE IN ALASKA NATIVE CHILDREN DUE TO SEROTYPES NOT IN THE HEPTAVALENT PNEUMOCOCCAL CONJUGATE VACCINE 2001-2005

T Cottle, T Cottle, TW Hennessy, L Bulkow, M Harker-Jones, D Hurlburt, D Parks, A Parkinson, CDC Arctic Investigations Program, Anchorage, Alaska, USA

BACKGROUND: Historically, rates of invasive pneumococcal disease (IPD) among Alaskan indigenous peoples (Alaska Natives (AN)) were among the highest reported and much higher than non-Native Alaskans. Since pneumococcal conjugate vaccine (PCV7) introduction in 2001, the rate of vaccine-type (VT) IPD among AN < 2 year-olds fell 94\% (275 cases/ 100,000 [1995-00] to 17 cases/100,000 [2004-5]). However, recent increases in non-VT IPD have been observed among AN.
METHODS: Cases were ill persons who had pneumococci isolated from a normally sterile site. Rates were obtained from statewide laboratory-based surveillance. Confirmed isolates were serotyped by the Quellung reaction. Proportions were compared using the chi-square test and trends in Poisson rates were evaluated with exact statistics.

RESULTS: Since 2001, non-VT IPD among AN < 2 year-olds has increased each year (38/100,000 in 2001 to 200/100,000 in 2005, $\mathrm{P}=0.001$ for trend). No significant increase in non-VT IPD was seen for AN 2-4 year olds or nonNative children $<5$ years old. Among AN $<2$ year-olds, six serotypes (3, $10 \mathrm{~A}, 15 \mathrm{~B}, 15 \mathrm{C}, 19 \mathrm{~A}, 38)$ caused an increased proportion of non-VT IPD since $\mathrm{PCV} 7$ use ( $20 \%$ prevaccine to $52 \%$ postvaccine, $\mathrm{P}=0.01)$. Among all ages and races, serotype 19A increased the most among non-VT isolates ( $7 \%$ prevaccine to $15 \%$ postvaccine, $\mathrm{P}=0.001$ ). Overall IPD rates for AN $<2$ year-olds initially decreased $72 \%$ from $403 / 100,000$ (prevaccine) to $112 / 100,000(2001-2)$ but have increased to $225 / 100,000$ in 2004-5, $\mathrm{P}<0.001$ (56\% of the prevaccine rate).

CONCLUSIONS: PCV7 use has been effective for preventing VT disease among AN. However, the increase in non-VT IPD rates among AN children under two years old have eroded gains made in IPD prevention through PCV7 use. If this trend continues, approaches other than PCV7 vaccine will be needed to prevent IPD in this population and to address the IPD disparity suffered by AN children.

\section{9 \\ VACCINATING ADOLESCENTS AGAINST MENINGOCOCCAL DISEASE IN CANADA: A COST- EFFECTIVENESS ANALYSIS}

P De Wals, Laval University; L Coudeville, Sanofi Pasteur; P Trottier, Université Laval; C Chevat, Sanofi Pasteur; LJ Erickson, AETMIS; VH Nguyen, Sanofi Pasteur

BACKGROUND: A tetravalent ACYW135 meningococcal polysaccharidediphtheria conjugate vaccine (4-MCV) has recently been licensed for use among persons aged 2 years and older. Effectiveness data from the UK and Spain indicate that the protection conferred by the meningococcal serogroup $\mathrm{C}$ conjugate vaccine $(\mathrm{C}-\mathrm{MCV})$ decreases with time, and there is a potential value for providing a booster dose during adolescence.

OBJECTIVES: To assess the cost-effectiveness of a booster dose at 12 years of age with either $\mathrm{C}-\mathrm{MCV}$ or $4-\mathrm{MCV}$ in addition to a first immunization at 1 year of age with C-MCV.

METHODS: A simulation model for assessing both the direct and indirect effects of vaccination was developed. Age- and serogoup-specific incidence rates and case-fatality rates were derived from Canadian surveillance data. Vaccine efficacy was estimated from UK and Spanish data, assuming an age-dependent decline of vaccine efficacy over time. Expected vaccine coverage rates were $90 \%$ at 12 months, and $70 \%$ at 12 years. Herd immunity was modeled using UK data. Vaccine purchase price per dose was $\$ 23$ for C-MCV, and $\$ 70$ for $4-\mathrm{MCV}$. Results, expressed in 2004 Canadian $\$$ and from a societal perspective, are presented for a steady state situation and a population size of 1 million (discount rate: $3 \%$ per year).

RESULTS: Under the "no vaccination" base scenario, 5.7 cases of vaccinepreventable meningococcal disease would occur each year in the study population, and total disease costs would be $\$ 735,170$. Implementing vaccination at 12 months using C-MCV would prevent 1.6 cases per year and reduce the burden of disease by $32 \%$. As compared to a strategy based on $\mathrm{C}-\mathrm{MCV}$ given at 1 year, adding a vaccination at 12 years of age could further reduce the number of cases by $55 \%$ if C-MCV was used and by $78 \%$ if 4-MCV was used. The impact on total net costs would be $-1 \%$ if a C-MCV was used and $+37 \%$ if a $4-\mathrm{MCV}$ was used. Efficacy of vaccination at 12 months and the differential price between the two vaccines are the parameters having the strongest impact on the cost/QALY ratios. Any increase in the incidence of serogroup Y similar to that recently reported for Ontario or the US improves the marginal cost-effectiveness ratio associated with 4-MCV.

CONCLUSION: Adolescent vaccination with either C-MCV or 4-MCV is cost-effective. Vaccination with C-MCV has a lower cost but is also less effective. 
O20

KINETICS OF THE SERUM IGG AND IGA ANTIBODY RESPONSE (ABR) IN HEALTHY WOMEN OF CHILDBEARING AGE AFTER IMMUNIZATION WITH TDAP B Halperin, Dalhousie University; SA McNeil, Dalhousie University; JM Langley, Dalhousie University; J Mutch, Dalhousie University; D Mackinnon-Cameron, Dalhousie University; SA Halperin, Dalhousie University

BACKGROUND: Adolescents and adults act as a primary source of pertussis infection for infants $<6$ months of age. A proposed method of protecting young infants is to immunize close contacts, thereby reducing transmission (cocoon strategy). For this to be effective, high levels of pertussis antibodies must be achieved rapidly following immunization. We sought to determine if the AbR to Tdap is sufficiently rapid to support the cocoon strategy.

METHODS: Healthy women 18-35 years of age were recruited $(n=30)$. Baseline serum IgG, serum IgA, and salivary IgA testing against pertussis toxoid (PT), filamentous hemagglutinin (FHA), pertactin (PRN), and fimbriae 2 and 3 (FIM) was performed, as well as baseline serum IgG antibody testing against diphtheria and tetanus. Baseline testing was followed by administration of a single dose of Tdap (ADACEL ${ }^{\text {TM }}$, Sanofi Pasteur). Serum and salivary antibodies were measured on days $1,2,3,5,7,14$, and 28 post-immunization.

RESULTS: A total of $83 \%$ (25/30) of women developed IgG antibodies against PT and PRN, while 90\% (27/30) and 97\% (29/30) responded against FHA and FIM respectively. An IgA response was elicited against PRN by $80 \%$ (24/30), FIM by $87 \%$ (26/30) and FHA by $63 \%(19 / 30)$ but against PT by only $10 \%(3 / 30)$ of participants. Serum IgG and IgA rose noticeably by day 7 , and approached their peaks by day 14 for all pertussis antigens except PT IgA.

CONCLUSIONS: Although the AbR to a booster dose of Tdap in healthy women of child-bearing age occurs by day 14 and is suggestive of an anamnestic immune response, it may not be sufficiently rapid to protect infants in the first weeks of life.

\section{O21}

\section{ATTITUDES, BELIEFS AND KNOWLEDGE ASSOCIATED WITH INCREASED UPTAKE OF MENINGOCOCCAL C (MENC) VACCINATION AMONG TORONTO HIGH SCHOOL STUDENTS}

O Kadri, Toronto Public Health; L Macdougall; E Gournis;

A Mathur; M Finkelstein

BACKGROUND: In 2005 the Ontario government funded a meningococcal serogroup $\mathrm{C}$ vaccine campaign targeted at youth born between January 1, 1985 and December 31, 1990. Immunization programs seldom target high school aged children, as most vaccinations occur in infancy. Given health behaviors begin early and receipt of MenC vaccination is elective, it is important to understand what motivates youth to receive this new vaccine. This information can be used to promote future public health promotion and prevention programs targeting youth.

OBJECTIVE: To identify factors related to the knowledge, attitudes and beliefs of youth associated with receiving MenC immunization.

METHODS: A school-based survey was conducted on a representative sample of Toronto District School Board high school students, aged 13 to 19 years. A questionnaire was administered to participants to collect information related to beliefs, knowledge and attitudes about MenC immunization. Univariate and bivariate analyses were carried out with SAS and deemed significant if $\mathrm{p}<0.05$.

RESULTS: The overall response rate for the survey was $71 \%$. MenC immunization was received by $64 \%(n=1141)$ of students with complete data $(n=1794)$. Factors significantly associated with increased vaccine uptake included: 1 ) experiencing peer pressure $(\mathrm{p}=0.0001), 2)$ feeling it was important to engage in healthy activities $(\mathrm{p}=0.02)$, and 3$)$ not fearing side-effects $(\mathrm{p}<0.02)$. The most frequent reasons reported for vaccine refusal were previous meningitis immunization $(34 \%)$ and preference for receiving the vaccine at family doctor's office $(27 \%)$.
CONCLUSION: Results confirm that youth are affected by their peer group and that youth already exhibiting healthier behaviours more readily accept vaccination. These results can be used to effectively promote future immunization programs and achieve higher immunization coverage levels among youth. Future health education and promotion campaigns aimed at students should focus on building awareness and acceptance in peer groups to ensure and improve their success with school-based immunization programs.

\section{O22}

VACCINE-PREVENTABLE EMPYEMA DUE TO COMMUNITYACQUIRED PNEUMONIA IN CANADIAN CHILDREN: A PEDIATRIC INVESTIGATOR'S COLLABORATIVE STUDY ON INFECTIONS IN CANADA (PICNIC) STUDY

Joanne Langley, Dalhousie University; J Kellner, University of Calgary; J Robinson, University of Alberta; N Le Saux, University of Ottawa; B Tan, University of Saskatchewan; R Ulloa-Gutierrez, University of British Columbia; S Dobson, University of British Columbia; U Allen, University of Toronto

BACKGROUND: Empyema is a serious complication of lower respiratory tract infection. We reviewed the epidemiology of pediatric empyema at 8 Canadian pediatric health centers to determine the percentage that could be vaccine preventable.

METHODS: Health records for children $<18$ years admitted from 1/1/00 to $30 / 12 / 03$ were searched for ICD-9 code 510 or ICD-10 code J869 (Empyema) and microbiology records reviewed for pleural fluid (PF) samples. Empyema was defined as least one of: thoracentesis with microbial growth from PF or no PF growth but compatible chemistry or cell count or radiologist diagnosis or diagnosis at surgery. Empyemas due to nonrespiratory illness (eg, chest trauma, thoracic surgery, esophageal rupture) were not eligible. Data were retrieved using a standard form with data dictionary. RESULTS: 251 children met inclusion criteria. Caucasians comprised $42 \%$; aboriginals $19 \%$, Asians $7 \%$, other $32 \%$. Most children were previously healthy $(95 \%)$. The age distribution was $<2$ years $=18 \%, 2$ to $<5$ years $=33 \%, 5$ years to $<10$ years $=24 \%, 10-17$ years $=24 \%$. Admissions occurred in all months with spring and late fall peaks. Ventilatory support occurred in 33\% (83/249) of children and 75\% (185/248) had chest tube placement. Organisms found in sterile samples (blood, PF, lung biopsy) were Streptococcus pneumoniae $(\mathrm{n}=39)$, and Streptococcus pyogenes $(\mathrm{n}=19)$, Staphylococcus aureus (15) of which 8 were methicillin-resistant, Mycobacterium tuberculosis (8), viral (2) and other (12). Of the $35 \mathrm{~S}$ pneumoniae isolates that were typed, $48 \%$ were due to serotypes present in the pneumococcal conjugate vaccine: serotype $14(\mathrm{n}=10), 6 \mathrm{~B}(\mathrm{n}=3), 9 \mathrm{~V}$ $(n=2), 23 F(n=1)$ and $4(n=1)$. Other serotypes were $1(n=8), 3(n=9), 6 \mathrm{~A}$ $(\mathrm{n}=1)$.

CONCLUSIONS: A significant proportion of empyema, a serious complication of pneumonia that occurs predominately in children under 5 years, is vaccine preventable.

\section{O23}

LONG TERM EFFICACY OF 23-VALENT PNEUMOCOCCAL POLYSACCHARIDE VACCINE (PPV) IN ONTARIO

Z Liu, Mount Sinai Hospital; KA Green, Mount Sinai Hospital; M Lovgren, National Centre for Streptococcus; G Tyrrell, National Centre for Streptococcus; A McGeer, Mount Sinai Hospital

INTRODUCTION: S pneumoniae is the most common cause of community-acquired pneumonia and bacteremia in adults. The National Advisory Committee on Immunization (NACI) recommends PPV for adults $>65 \mathrm{y}$, and those aged $>2 \mathrm{y}$ with immunocompromising conditions. We used the indirect cohort method to estimate vaccine efficacy (VE) in different adult populations.

METHODS: Population-based surveillance in metropolitan Toronto and Peel Region for invasive pneumococcal disease (IPD) from 1/1995 and for culture confirmed non-bacteremic pneumococcal pneumonia (NBPP) from 1/2002. Isolates serotyped at National Center for Streptococcus. Underlying disease categorized as immunocompromising or not. Vaccine status prior to infection obtained via medical records, patient and physician interview. VE estimated using indirect cohort method. 
RESULTS: Of 3138 adult IPD episodes, 2615 occurred in persons eligible for PPV. For 2312 (82\%) isolates/serotypes were available; for 1660 of these $(73 \%)$ vaccination history was available. Overall, $1453(87 \%)$ of episodes were caused by vaccine-type strains: the most common serotypes were: 14 ( $\mathrm{N}=274,17 \%), 3$ (160, 9.6\%), 4 (142, 8.6\%), 6B (137, 8.3\%), and $23 \mathrm{~F}(132,8.0 \%) .1246$ episodes $(75 \%)$ occurred in immunocompetent patients. VE against IPD was 50\% (95\% CL 33\%, 63\%):52\% (32\%, 67\%) for immunocompetent patients and $41 \%(-1 \%, 67 \%, \mathrm{P}=0.07)$ for immunocompromised patients. VE did not decrease with increasing age. There was no evidence of waning VE over time: VE was $54 \%(32 \%, 68 \%)$ for vaccine received $<3$ y previously, $47 \%(0,60 \%)$ for vaccine received $3-6 y$ previously, and $64 \%(35 \%, 80 \%)$ for vaccine received $>6$ y prior. There were 341 episodes of NBPP from 2002 to 2004; 295 in adults eligible for PPV. Of these, 250 (85\%) had isolates/serotypes available, and 165 of these $(66 \%)$ had vaccination history available. 122 (74\% of episodes were caused by vaccine-type strains; the most common serotypes were: $3(\mathrm{~N}=27$, $16 \%), 19 \mathrm{~F}(25,15 \%), 6 \mathrm{~B}(16,9.7 \%)$ and $6 \mathrm{~A}(13,7.9 \%)$. Estimated VE against NBPP was $16 \%$ (95\% CI -29\%, 58\%).

CONCLUSION: PPV is clearly efficacious against IPD in eligible adult recipients, and likely provides protection even for immunocompromised patients. Re-vaccination at $<10$ years does not appear warranted. We found no evidence for PPV protection against NBPP, which may be in part due to serotype differences between NBPP and IPD.

\section{POSTER PRESENTATIONS Bacterial Diseases and Vaccines}

\section{P1}

\section{MENINGOCOCCAL INFECTIONS IN ADULTS ACROSS CANADA, 2002-2005}

J Bettinger, Vaccine Evaluation Center; N Le Saux, University of Ottawa, Ottawa, Ontario; DW Scheifele, Vaccine Evaluation Center, University of British Columbia, Vancouver, British Columbia; SA Halperin, Clinical Trials Research Center, Dalhousie University, Halifax, Nova Scotia; K Marty, Vaccine Evaluation Center, Vancouver, British Columbia; R Tsang, National Microbiology Laboratory, Winnipeg, Manitoba

BACKGROUND: The epidemiology of meningococcal infections is crucial to planning effective immunization programs, yet it varies significantly by age, place and time. Within a Canada-wide surveillance project, we examined the influences of age, known risk factors, serogroup and geographic location on adult infections.

METHODS: Active metropolitan area surveillance for meningococcal infections in adults was conducted by the 12 centers of the Immunization Monitoring Program, Active (IMPACT) from 2002-2005. Surveillance occurred within defined urban areas of each IMPACT center, which included almost half of the Canadian adult population. Inclusion required a sterile site isolate. Isolates were serogrouped at the National Microbiology Laboratory. 2004 population census estimates were used for incidence rate calculation.

RESULTS: 164 cases were reported in adults > 20 years: 55 in 2002, 34 in 2003, 46 in 2004 and 29 in 2005. Overall, 55\% were female: 54\% had an underlying health condition, $9 \%$ were immunocompromised and $52 \%$ had a known risk factor for meningococcal infection (most often smoking). Case fatality was $10 \%$. Average annual incidence was 0.3 per 100,000 (range 0.2-0.4) overall, but varied from 0.8 (95\% CI 0.5, 1.1) in 20-24 year olds to $0.2(0.1,0.4)$ in $55-59$ year olds during the surveillance period. Most infections were serogroup C (37\%), followed by B (34\%) and Y (23\%). Serogroups were distributed geographically as follows: serogroups B (66\%) and C (33\%) in Halifax/St Johns; A (1\%), B (38\%), C (27\%), W135 (5\%) and Y (25\%) in Quebec and Ontario; C (75\%) and Y (25\%) in Winnipeg; and B (30\%), C (47\%) and Y (21\%) in Alberta and British Columbia.

CONCLUSIONS: Significant variability existed in the distribution of serogroups across Canada. Serogroups C and B remained the most common followed by Y. The highest incidence occurred in 20-24 year olds. This information can be used to guide immunization programs.

\section{P2 \\ APPROPRIATELY VACCINATED CHILDREN AMONG HOSPITALIZED PERTUSSIS CASES}

J Bettinger, Vaccine Evaluation Center; SA Halperin, IWK Health Center and Dalhousie University, Halifax, Nova Scotia; G De Serres, Quebec Institute of Public Health, Quebec, Quebec; DW Scheifele, University of British Columbia, Vancouver, British Columbia; T Tam, Public Health Agency of Canada, Ottawa, Ontario

INTRODUCTION: Because whole cell pertussis vaccine (WCV) used in Canada had lower effectiveness than the acellular vaccine (ACV) introduced in 1997-1998, we compared the proportion of appropriately vaccinated children by age and vaccine among children hospitalized with pertussis from 1991-2004.

METHODS: We analyzed the Canadian Immunization Monitoring Program Active (IMPACT) database for all pertussis cases from January 1991 - December 2004. IMPACT is an active surveillance network in 12 Canadian pediatric centers. Pertussis occurring after 3 or 4 vaccinations, starting $>14$ days after the last immunization defined an appropriately vaccinated case. We excluded cases without vaccine information. Fisher's exact test was used for comparisons.

RESULTS: 2,091 children were admitted with pertussis. Vaccine information was available for 1,595: 1,305 were $<6$ months and age-ineligible for 3 doses, while 290 were 6 months -16 years. The overall proportion of cases aged 6-23 months who received $>3$ doses was $62 \%(157 / 255)$. In those $>24$ months $77.1 \%(27 / 35)$ had received $>4$ doses. The highest proportion of cases after 4 doses occurred among the 10 to 16 year age group with $94 \%(17 / 18)$, followed by $64 \%$ (7/11) of $5-9$ year olds, and $50 \%(3 / 6)$ of $2-4$ year olds. In children 6-23 months, the proportion of cases after $>3$ doses was significantly higher with WCV (53\%) than ACV (7\%) $(\mathrm{p}=0.05)$.

CONCLUSIONS: ACV provided superior protection after 3 doses in 6-23 month olds when compared to WCV. Hospitalizations in 6-23 month olds declined since the introduction of ACV, implying greater ACV effectiveness. After 4 doses, WCV provided good protection, permitting few hospitalized cases in 2-16 year olds. The greatest proportion of fully vaccinated cases among hospitalized pertussis patients occurred in older ages vaccinated with WCV, implying waning immunity.

\section{P3}

\section{IMPACT OF MASS VACCINATION USING 23-VALENT POLYSACCHARIDE PNEUMOCOCCAL VACCINE TO CONTROL AN EPIDEMIC OF SEVERE PNEUMONIA IN NUNAVIK}

P De Wals, Laval University; AA Ndiaye, Laval University; JF Proulx, Nunavik Public Health Directorate; M Ouakki, Quebec University Hospital Research Centre; L Jetté, Quebec Public Health Laboratory; S Déry, Nunavik Public Health Directorate BACKGROUND: In the spring of 2002, a mass immunization campaign using a 23-valent pneumococcal polysaccharide vaccine (23-PPV) was launched in order to control an outbreak of severe pneumonia caused by a virulent clone of serotype 1 Streptococcus pneumoniae in Nunavik, Quebec, Canada.

OBJECTIVES: To evaluate the impact of the campaign on the incidence of invasive pneumococcal disease (IPD) and hospitalizations possibly associated with pneumococcal infections (HPAPI) in the mostly Inuit population aged 10 to 64 years.

STUDY DESIGN: Retrospective analysis of laboratory surveillance and hospital administrative data.

STATISTICAL ANALYSES: Multivariate Poisson model comparing the frequency rates of selected outcomes before the outbreak, during the outbreak, and after the implementation of the mass immunization program. RESULTS: At the end of the vaccination campaign, $84 \%$ of the target population $(n=10,284)$ had been vaccinated. Compared with the epidemic period, the reported incidence of serotype 1 IPD decreased markedly after the implementation of the vaccination campaign (rate ratio $=0.16$; $p<0.002$ ). The frequency of HPAPI and the mean duration of hospital stay also decreased. However, vaccine failures were documented and the 
HPAPI baseline rates after the campaign remained equal or higher than in the period prior to the outbreak.

CONCLUSION: Although 23-PPV contributed to control the outbreak, better vaccines are needed for the prevention of sporadic infections and outbreaks caused by serotype 1 pneumococcus. The currently available 7-valent pneumococcal conjugate vaccine does not contain the serotype 1 antigen. Nine- or 11-valent pneumococcal conjugate vaccines not yet licensed contain the serotype 1 antigen, but there is no data on their effectiveness to protect against invasive and non-invasive infections caused by serotype 1 pneumococcus. A collaborative study is urgently needed.

\section{P4}

MORBIDITY AND MORTALITY OF INVASIVE MENINGOCOCCAL DISEASE IN CANADA 2002-2005

N Le Saux, Division of Infectious Diseases; J Bettinger, University of British Columbia; S Halperin, Dalhousie University; D Scheifele, University of British Columbia; K Marty, University of British Columbia; R Tsang, University of Manitoba

BACKGROUND: The mortality and morbidity of invasive meningococcal disease (IMD) have a high likelihood of being reduced by immunization. OBJECTIVE: To determine mortality and morbidity for IMD in adults and children prior to implementation of widespread immunization programs. METHODS: Population-based surveillance for IMD was conducted in 12 centers of the Immunization Monitoring Program, Active (IMPACT) program from 2002 to 2005.

RESULTS: 345 patients (181 were less than 19 years) had IMD. 26 patients ( 9 children and 17 adults) succumbed, a case fatality rate (CFR) of $8 \%$. The CFR was $4.9 \%$ for those less than 19 years and $10.4 \%$ for those over 20 years $(\mathrm{p}=0.06)$. The CFR for patients whose presentation included shock, meningitis, shock and meningitis or other bacteremia was $8 \%$ (9/107), $0 \%(0 / 212), 25 \%(16 / 63)$, and $1 \%(1 / 77)$ respectively. There was no significant association between serogroup and mortality, although $\mathrm{B}$ and C cases occurred most frequently. 205 patients (59.4\%) were admitted to intensive care. The median duration of hospital stay was 8 days $(95 \%$ range 1-24). Of 319 patients who survived, sequelae occurred in at least $17.5 \%$ (56); $4.7 \%$ (15) required amputation; $5.3 \%$ (17) had cicatricial scarring; 5\% (16) had hearing deficits; 2.8\% (9) had other neurological sequelae and $2.5 \%$ (8) had renal dysfunction. Sequelae occurred in $16 \%$ $(17 / 107)$ of patients with manifestations that included shock and in $4 \%$ (8/212) with meningitis. Of patients with sequelae, the median duration of hospital stay was 15.5 days (range 5-107). 19.6\% (11) of patients required transfer to rehabilitation facilities.

CONCLUSIONS: The overall CFR of $8 \%$ for IMD is comparable to other reports and was highest in patients who presented with both shock and meningitis. Over $50 \%$ of patients required admission to ICU. Sequelae occurred in $17 \%$ of those who survived and were more common in those with shock.

\section{P5}

\section{EVALUATING FACTORS THAT FAVOUR RETENTION OF SUBJECTS IN PEDIATRIC VACCINE TRIALS}

C LaJeunesse, Vaccine Evaluation Center; A Kallos; K Marty; M Mozel; D Scheifele

BACKGROUND: Retention of subjects in clinical trials is important as losses imperil interpretation of findings and attainment of study objectives. Safeguarding the health of individuals and respecting their rights and dignity are necessary precautions in conducting research in humans ("Good Clinical Practice", GCP) but is this enough to encourage continued participation? We developed a "Satisfaction Survey" to assess subjects' experiences as research participants.

METHODS: A questionnaire was developed using Likert response scales to assess logistical issues during study participation (eg parking, wait times, courtesy of research staff) and GCP issues (eg participants were well informed about the study, understood study requirements at entry, were shown respect for their rights, dignity, and safety). The instrument was applied to parents of toddlers who were completing a 7 month pre-licensure vaccine study, which had a $98 \%$ retention rate to the final visit.
RESULTS: The questionnaire was completed for $98 \%$ of subjects (169/172). 98\% rated the experience as positive for their child and 96\% said that they would consider participating in another study. Virtually all agreed that each of the key GCP requirements had been met to their satisfaction. CONCLUSION: The site's compliance with Good Clinical Practices clearly favours retention of study participants and preserves their willingness to consider future study participation. The survey instrument provides reassuring (but otherwise unavailable) information for the study team, ethics committee and sponsor and is now being used routinely in all of our vaccine studies, with all age groups.

\section{P6}

THE BURDEN OF PNEUMOCOCCAL DISEASE IN THE CANADIAN POPULATION PRIOR TO ROUTINE USE OF THE 7-VALENT PNEUMOCOCCAL CONJUGATE VACCINE

A Morrow, Université Laval; P De Wals, Laval University; G Petit, University of Montreal; M Guay, University of Sherbrooke;

LJ Erickson, Quebec Agency for Health Services and Technology Assessment

BACKGROUND: In the United States, implementation of the 7-valent conjugate vaccine into childhood immunization schedules has had an effect on the burden of pneumococcal disease in all ages of the population. In order to evaluate the impact in Canada, it is essential to have an estimate of the burden of pneumococcal disease before routine use of the vaccine.

METHODS: The incidence and costs of pneumococcal disease in the Canadian population in 2001 were estimated from various sources, including published studies, provincial databases, and expert opinion.

RESULTS: In 2001, there was 565,000 cases of pneumococcal disease in the Canadian population, with invasive infections representing $0.7 \%$ of cases, pneumonia $7.5 \%$, and acute otitis media $91.8 \%$. There was a total 3000 deaths, mainly as a result of pneumonia and largely attributable to the populations over 64 years of age. There was 54330 life-years lost due to pneumococcal disease, and 37,430 quality-adjusted life-years lost due to acute disease, long-term sequelae and deaths. Societal costs were estimated at $\$ 193$ million (range: $\$ 155$ to $\$ 295$ ), with $82 \%$ borne by the health system and $18 \%$ borne by families. Invasive pneumococcal infections represented $17 \%$ of the costs, and non-invasive infections represented $83 \%$, with approximately half of this proportion attributable to acute otitis media and myringotomy.

CONCLUSION: The burden of pneumococcal disease before routine use of the pneumococcal conjugate vaccine was substantial in all age groups of the Canadian population. This estimate will provide a baseline for further analysis of the direct and indirect impact of the vaccine.

\section{P7}

NON-TYPEABLE HAEMOPHILUS INFLUENZAE: A MAJOR/COMMON CAUSE OF INVASIVE HAEMOPHILUS INFLUENZAE DISEASE

M Sill, Public Health Agency of Canada; S Skinner, University of Manitoba; J Wylie, Cadham Provincial Public Health Laboratory; D Law, Public Health Agency of Canada, National Microbiology Laboratory; J Zhou, Public Health Agency of Canada, National Microbiology Laboratory; R Tsang, Public Health Agency of Canada, National Microbiology Laboratory

INTRODUCTION: We are carrying out an in-depth prospective study in the province of Manitoba to collect invasive $\mathrm{Hi}$ isolates and the clinical data associated with these cases. In this abstract, we present our preliminary data collected during a 15 month period from May 2005 to July 2006. METHODS: The Hi isolates were identified by standard biochemical tests, serotypes were determined by bacterial agglutination test and PCR, and genetic studies were done by MLST and PFGE. Susceptibility to antibiotics was determined by disk diffusion test. Clinical data were obtained by reviewing the patients' charts/records.

RESULTS: Thirty isolates were collected: $67 \%$ (20 isolates) were nontypeable (NT) while 20\% (6 isolates) belonged to serotype a: Two serotype f, 1 serotype $\mathrm{b}$ and 1 serotype e were also identified: Two of the invasive NT-Hi cases were found in newborns $(<7$ days old), three were in the age group of 
2 to 4 years old, and the remaining 15 cases were found in adults (ages ranged from 19 years to 90 years). The clinical picture of NT-Hi disease involving 15 cases (including some of our previously published cases identified during the period of 2000-2004 in Manitoba) had 6 patients with sepsis, 5 pneumonia, 3 meningitis and one pelvic abscess. Four patients had underlying pulmonary disease and 3 were immunocompromised. The overall mortality rate was $27 \%(4 / 15)$.

CONCLUSION: As a result of implementation of universal vaccination against $\mathrm{Hi}$ type b, invasive $\mathrm{Hi}$ disease in Manitoba is now mostly due to serotypes other than serotype b. In the present study, over $50 \%$ of the cases were caused by non-typeable strains and clinically NT-Hi cases were associated with severe disease. Future surveillance of invasive $\mathrm{Hi}$ disease should include all Hi isolates regardless of their serotype nature.

\section{P8}

\section{IMMUNOLOGIC CONSIDERATIONS FOR THE TIMING OF THE BOOSTER (FOURTH) DOSE OF 7-VALENT PNEUMOCOCCAL CONJUGATE VACCINE (PCV7) IN CHILDREN}

D Scheifele, Vaccine Evaluation Center; SA Halperin, Dalhousie University, Halifax, Nova Scotia; JJ Ochnio, Vaccine Evaluation Center, The University of British Columbia; D Duarte-Monteiro, Wyeth Pharmaceuticals, Markham, Ontario; D Wortzman, Wyeth Pharmaceuticals, Markham, Ontario

INTRODUCTION: The PCV7 booster dose is recommended at age 12-15 months but delaying it to 18 months would better fit the routine schedule used in most provinces, provided that adequate antibody concentrations persist.

METHODS: Subjects had received 3 primary PCV7 doses (at ages 2, 4 and 6 months) and been bled at age 7-8 months to assess responses. They were re-enrolled at age 12 months, bled and randomly assigned to receive a PCV7 booster dose at 15 or 18 months, with serotesting just before and 4 weeks after re-vaccination. Antibody concentrations for each serotype were measured and geometric mean concentrations (GMCs) compared at $7-8,12,15$ or 18 months and post-booster.

RESULTS: 335 children participated, with 167 boosted at 15 months and 164 at 18 months. Antibody concentrations declined substantially between $7-8$ and 12 months but minimally thereafter. GMCs at 15 months averaged $23.4 \%$ of the peak values at $7-8$ months and $19.8 \%$ of those peak values at 18 months. Antibody decreases were similar for 6 types but type 14 antibodies had a higher GMC at 12 months because $14 \%$ of subjects had spontaneous titer increases after 7-8 months, likely from colonization. This subset had a lower GMC at 7-8 months than those without subsequent spontaneous increases ( 1.8 vs $6.7 \mu \mathrm{g} / \mathrm{mL}$, respectively, $\mathrm{p}<0.01)$. Booster responses were brisk, with no significant differences in antibody concentrations by age for any serotype. Mild injection site redness and swelling were less frequent at 18 than 15 months.

CONCLUSIONS: Pre-booster antibody concentrations differed little between 15 and 18 month age groups and both responded strongly, suggesting that either age may be considered for booster vaccination.

\section{P9}

\section{INVASIVE BACTERIAL RESPIRATORY DISEASE IN THE CANADIAN NORTH}

S Deeks, Public Health Agency of Canada; N Degani, Immunization \& Respiratory Infections Division, PHAC; T Cottle, Arctic Investigations Program, Centers for Disease Control; R Carlin, Direction de santé publique des Territoires Cris de la Baie-James; C Case, Health \& Social Services, Northwest Territories; C Hemsley, Yukon Health \& Social Services, Yukon; C Palacios, Department of Health \& Social Services, Nunavut; JF Proulx, Direction de santé publique du Nunavik, Québec; M Lovgren, National Centre for Streptococcus; L Jetté, Laboratoire de santé publique du Québec; R Tsang, National Microbiology Laboratory, PHAC; PM Roberts, Health \& Community Services, Labrador Region, Newfoundland and Labrador, C Navarro, Immunization \& Respiratory Infections Division, Public Health Agency of Canada, Ontario

INTRODUCTION: The International Circumpolar Surveillance Network has conducted population-based surveillance of invasive disease caused by Streptococcus pneumoniae (Sp) and Haemophilus influenzae (Hi) since 1999 and 2000, respectively. Canadian regions involved are Yukon, Northwest Territories, Nunavut, and northern regions of Quebec and Labrador. The total population is 132,956; 59\% are Aboriginal. While all regions have universal $\mathrm{Hi}$ type $\mathrm{b}(\mathrm{Hib})$ vaccination programs, universal pneumococcal conjugate vaccine (pcv7) programs were implemented during the surveillance period.

METHODS: Standardized clinical and demographic information were collected. Bacterial isolates were forwarded to reference laboratories for confirmation and serotyping.

RESULTS: Between 1999-2005, 251 cases of Sp were reported. Crude annual incidence ranged from 17.3 (2005) to 38.4 (2001) cases per 100,000 population. Children $<2$ years had the highest incidence. Crude annualized incidence among Aboriginals compared to non-Aboriginals was 36.0 vs 9.9 per 100,000 population/year. Based on age and serotype analysis, $59.6 \%$ of cases among children $<2$ years were preventable with pcv 7 and $88.5 \%$ of cases among adults $>65$ years were preventable with 23 -valent vaccine. In the 2 regions that implemented universal pcv7 programs, there were 19 cases of preventable illness in children $<2$ years before program implementation (1999-2002) and none after. This was significantly lower than the number reported in the remaining regions (Fisher's exact p value $<0.03$ ). Between 2000-2005, $62 \mathrm{Hi}$ cases were reported; $53 \%$ were type a. Crude annual Hi incidence ranged from 4.5 (2003) to 13.5 (2001) cases per 100,000 population. Crude annualized incidence among Aboriginals compared to non-Aboriginals was 10.1 vs 1.3 per 100,000 population/year. Of the $8 \mathrm{Hib}$ cases, 2 were unvaccinated, 4 received one dose and 2 had unknown status. Six were in infants $<6$ months.

CONCLUSION: Invasive bacterial respiratory diseases are important causes of morbidity among northern Canadians. Aboriginal people are particularly impacted.

\section{P10}

\section{INVASIVE MENINGOCOCCAL C DISEASE IN CANADA, 1995-2004 - AN INITIAL LOOK AT IMMUNIZATION PROGRAM IMPACT}

C Navarro, Public Health Agency of Canada (PHAC); K Watkins, Immunization \& Respiratory Infections Division, PHAC, Ottawa, Ontario; SL Deeks, Immunization \& Respiratory Infections Division, PHAC, Ottawa, Ontario; A Medaglia, Immunization \& Respiratory Infections Division, PHAC, Ottawa, Ontario; R Tsang, National Microbiology Laboratory, PHAC, Winnipeg, Manitoba; T Tam, Immunization \& Respiratory Infections Division, PHAC, Ottawa, Ontario

INTRODUCTION: Meningococcal C conjugate (Men-C C) vaccine was approved for use in Canada in 2001. The National Advisory Committee on Immunization recommends the vaccine for all children under 5 years, adolescents and young adults. Between 2002 and 2004, 10 jurisdictions had implemented universal Men-C programs at various 
ages. In June 2005, recommendations for the reduction of invasive meningococcal disease (IMD) were developed during a national consensus conference, taking into account the expected impact of the new vaccine. METHODS: Provinces/Territories report non-nominal epidemiologic data to the Public Health Agency of Canada on all IMD cases meeting the national case definition. The National Microbiology Laboratory confirms serogroup and conducts further bacteriologic studies.

RESULTS: IMD is endemic in Canada with periods of increased activity every 10 to 15 years. Incidence is highest among children under 1 year and declines as age increases except for a smaller peak in the 15 to 19 year age group. Serogroups B and C have been responsible for most cases in Canada. Serogroup C has almost exclusively been responsible for outbreaks. Serogroup C disease has a higher case fatality ratio (mean 13\%) and, prior to universal immunization programs, a greater incidence in adolescents compared to serogroup B. The hypervirulent clone of ET-15 (with the serotype antigen 2a) is responsible for most serogroup C IMD reported in Canada. The average incidence for serogroup C disease during 1995-2001 and 2002-2004 was 0.31 per 100,000 and 0.19 per 100,000 , respectively. The average incidence declined $39 \%$ between the two periods.

CONCLUSION: Preliminary data suggest that the national recommendation to achieve a sustained reduction of $70 \%$ in the incidence of serogroup C disease by 2010 is achievable with increased implementation of routine Men-C $\mathrm{C}$ vaccination programs in Canada. Continued enhanced surveillance will be required to monitor progress towards recommended targets.

\section{P11}

\section{VACCINE PRESSURE AND IMMUNE SELECTION ON VACCINE PREVENTABLE BACTERIAL AGENTS}

R Tsang, National Microbiology Laboratory; D Law, National Microbiology Laboratory; M Sill, National Microbiology Laboratory; A Henderson, National Microbiology; M Blake, National Microbiology Laboratory; J Stoltz, National Microbiology Laboratory; I Martin, National Microbiology Laboratory

INTRODUCTION: Immune selection on bacteria may result from natural immunity developed in the population as a result of endemic disease or from vaccine pressure. This study looks at immune selection on a few vaccine preventable bacterial disease agents.

\section{RESULTS:}

Immune Selection from Vaccine Pressure

- In Streptocuccus pneumoniae causing invasive pneumpcoccal disease, introduction of the heptavalent pneumococcal conjugate vaccine (PCV7) has led to the emergence of diseases due to either vaccine-related pneumococcal serotypes or nonvaccine serogroupr. Gapsule sn ltching has also been demonstrated in disease-causing pneung pecocer.

- Changes in the epidemiology of invad Naenophilus influenzae disease have been reported from different ge thes Including Canada, in the post $\mathrm{H}$ influenzae serotype b (Hib) vardination era.

- In Bordetella pertussis, man genetic polymorphisms have been described, involving such diy rse dnes like fimbriae, pertussis toxin, and pertactin that encode for the vinulence factors (and hence vaccine components). Analysis of genomic DNA from B pertussis strains by pulsed-field gel electrophoresis is also able to differentiate between recent clinical isolates and strains used in the manufacturing of pertussis vaccines. These genetic changes have been suggested to be responsible for the resurgence of pertussis in countries with high vaccine coverage.

Immune Selection from Natural Immunity

- After more than a decade of endemic disease and many localized outbreaks, genetic and antigenic changes of sub-capsular protein antigens (involving the serotype and serosubtype antigens as well as the iron-regulated outer membrane proteins such as FetA) were detected in the serogroup C ET-15 Neisseria meningitidis strains. These genetic changes causing antigenic shifts may explain increased disease activities in the early 2000 in Canada.

CONCLUSION: Since bacteria change over time, and some of these changes may lead to vaccine escape mutants, it is essential for public health laboratories to have a system to monitor the evolving bacteria for causing potential vaccine breakthrough cases.

\section{P12}

IMPACT OF ACELLULAR PERTUSSIS VACCINE FOR CHILDREN AND TEENS IN CANADA: SIMULTANEOUS COHORT EFFECTS IN BRITISH COLUMBIA

D Skowronski, BC Centre for Disease Control; SA Tweed, BC Centre for Disease Control; G De Serres, Universite Laval; S Halperin, IWK Health Centre; A Coombs, Nova Scotia Department of Health; J Isaac-Renton, BC Centre for Disease Control INTRODUCTION: Beginning in 1997, acellular pertussis (aP) replaced whole cell (wP) vaccine for Canadian children. Among pre-teens who previously received $\mathrm{wP}$ vaccine, a cohort effect of increased pertussis incidence, peaking at 9-11yrs, was identified in British Columbia (BC) in 2000, and also in Quebec (QC) and elsewhere: aP booster was added at 14-16yrs in BC and QC in 2004, and across Canada by 2005. We examine pertussis trends since 2000 in BC, QC and Nova Scotia (NS).

METHODS: Age-specific incidence was derived from surveillance data. In $\mathrm{BC},>90 \%$ of laboratory-confirmation was by both polymerase chain reaction (PCR) and culture at the BC Centre for Disease Control. In QC, diagnosis was primarily by culture; one tertiary-pediatric hospital used PCR alone. In NS, either PCR or culture was applied at various hospital settings.

RESULTS: The previously-identified cohort effect of increased incidence, advancing annually among pre-teens, persisted in all three provinces. Instead of the expected peak in children at 14-16yrs, a precipitous drop in incidence was observed in 2004-05 in all three provinces, signaling early impact of the adolescent program. In $\mathrm{BC}$ alone, pertussis rates in pre-teens exceeded rates in infants. A second cohort effect of lower incidence among children receiving more aP doses also emerged in BC. In 20002005, children who received $5 \mathrm{wP}$ doses (aged 9-14yrs) comprised 2-3-fold more pertussis reports than children receiving $1 \mathrm{aP}$ dose $(6-11 \mathrm{yrs})$ and 4 -15-fold more than children receiving $\geq 2 \mathrm{aP}$ doses (2-9yrs). Lower risk in children receiving $5 \mathrm{aP}$ doses extended at least $8-10 \mathrm{yrs}$. Early childhood trends were less evident in QC/NS.

CONCLUSIONS: aP vaccine has dampened the cohort effect of increased adolescent susceptibility and is driving a second cohort effect of decreased susceptibility and prolonged protection among younger BC children who received at least one dose. Consistent application of laboratory approaches across the lifespan, especially PCR with its better sensitivity compared to culture, is needed. This would improve the resolution and interpretation of age-related trends derived from surveillance data at the provincial and national levels.

\section{P13}

\section{CASE CONTROL STUDY OF A PERTUSSIS OUTBREAK IN TORONTO FROM OCTOBER 2005-MARCH 2006}

V Waters, Hospital for Sick Children; F Jamieson, Ontario Ministry of Health; L Ford-Jones, Hospital for Sick Children; S Richardson, Hospital for Sick Children; M Finkelstein, Toronto Public Health; S Halperin, IWK Health Center

BACKGROUND: Despite widespread immunization programs, a pertussis outbreak, the largest in at least 12 years, occurred in Toronto, Canada from October 2005 to March 2006.

METHODS: A retrospective case control study was initiated to identify the risks factors for being polymerase chain reaction (PCR) positive for Bordetella pertussis in children 0-18 years of age in Toronto from October 2005 to March 2006. Cases were age, geographic location and time of testing matched (1:2) to PCR negative controls. Information was obtained from Toronto Public Health, medical charts and telephone interviews. Conditional logistic regression was used to estimate the matched odds ratio using two-sided $95 \%$ confidence intervals.

RESULTS: 286 children were positive for B pertussis by PCR (3\% culture positive) in Toronto from October 2005 to March 2006. Data has been gathered on 127 cases to date. The median age of cases was $2.2 \mathrm{yrs}$ and $70 \%$ of cases were between 1-4 yrs of age. The majority had no pre-existing medical condition $(77 \%)$ and attended either school or daycare (53\%). Most of the cases $(57 \%)$ had visited a healthcare setting in the 30 days prior to the onset of illness. $62 \%$ of cases had a cough and at least one symptom suggestive of 
pertussis although only 3 cases (2\%) were hospitalized. Approximately one-quarter of cases had at least one household member with pertussis-like symptoms in the month before $(22 \%)$ or after $(17 \%)$ the case tested positive. CONCLUSIONS: The majority of cases were in previously well preschool children. Many cases did not meet a clinical case definition of pertussis suggesting they may have had attenuated disease. Further analysis will determine if exposure to school, daycare or healthcare setting was a risk factor for being $B$ pertussis PCR positive during this outbreak.

\section{P14}

PENTA- AND HEXAVALENT DTAP-BASED VACCINES APPROVED FOR USE IN CANADA: A SYSTEMATIC LITERATURE REVIEW OF IMMUNOGENICITY AND REACTOGENICITY

V Gilca, Laval University Hospital Center; B Duval, Institut National de Santé Publique du Québec; SH Deeks, Public Health Agency of Canada

BACKGROUND: Combination vaccines are widely accepted as the most efficient means of administering large numbers of antigens. In Canada, one hexa- and three pentavalent DTaP-based vaccines produced by two manufacturers are approved for use in infants and young children. The use of one combination vaccine in a country increases the risk of shortages. However, issues of interchangeability and comparable immunogenicity and safety are important.

OBJECTIVES: To conduct a systematic review of published results on immunogenicity and reactogenicity of: Pentacel ${ }^{\mathrm{TM}}$, Pediacel $^{\mathrm{TM}}$ (Sanofi Pasteur), Ifanrix ${ }^{\mathrm{TM}}$-IPV-Hib, and Infanrix ${ }^{\mathrm{TM}}$-hexa (GSK).

METHODS: A systematic search (1966-2006) of MEDLINE and EMBASE was conducted using the keywords "Quadracel", "Pentacel", "Pediacel", "Infanrix", and "DTaP-Hib-IPV or DTaP-HBV-IPV-Hib or DTaP-IPV-Hib or DTaP-IPV-HBV-Hib."

RESULTS: 206 references were retrieved. After exclusion of repeatedly cited references and non clinical trial reports, full texts of 71 articles were reviewed. The 20 articles reporting 22 clinical trial results on immunogenicity and/or reactogenicity of vaccines of interest were retained for this report. Important variation in immunogenicity results reported after a primary vaccination course was observed among published studies. The vaccination schedule used seems to be the most important factor in this variation. A trend towards lower immunogenicity was observed in studies using a compressed schedule. Very similar and encouraging immunogenicity results were reported after a booster dose of all vaccines included in the analysis. No clinically important differences in vaccine reactogenicity were observed, although data comparison is difficult because of the different approaches used in data collection and interpretation. No head to head study of vaccines produced by different manufacturers has been published. CONCLUSION: No evidence of clinically important differences in immunogenicity or reactogenicity of the different combination vaccines could be concluded from the available published data. Head to head studies are needed.

\section{P15}

IMMUNOGENICITY OF THE PNEUMOCOCCAL CONJUGATE VACCINE IN PAEDIATRIC SOLID ORGAN TRANSPLANT RECIPIENTS

M Barton, Division of infectious Diseases; S Wasfy, Division of Infectious Diseases; G Pikula, Division of Infectious Diseases; A Dipchand, Division of Cardiology; D Hebert, Division of Nephrology; V Ng, Division of Gastroenterology; M Solomon, Division of Respiratory Medicine; A Fecteau, Department of Surgery; U Allen, Division of Infectious Diseases

INTRODUCTION: Pneumococcal conjugate vaccines have been shown to induce adequate immune responses in normal hosts. Immunogenicity data are limited for high-risk populations such as transplant recipients. OBJECTIVES: 1) To determine the vaccine responses to the 7-valent conjugate pneumococcal vaccine in paediatric solid organ transplant (SOT) recipients. 2) To determine the antibody responses to the polysaccharide vaccine when used after the conjugate vaccine.
METHODS: SOT recipients were prospectively enrolled at $\geq 4$ months following transplantation. Pneumococcal vaccine-naïve subjects were eligible. They received 3 doses of Prevnar ${ }^{\mathrm{TM}}$ at 8-12 week intervals followed $8-12$ weeks later by one dose of the 23 -valent vaccine. Serology was done at baseline, 8-12 weeks following doses 2 and 3 of Prevnar ${ }^{\mathrm{TM}}$ and following the polysaccharide vaccine. Serology was done by ELISA.

RESULTS: Eighty-two recipients were enrolled at a median age of $7.9 \mathrm{yrs}$ (range $0.6-18$ yrs). There were 31 heart, 27 kidney, 19 liver and 5 lung recipients. The median time from transplantation to initiation of immunization was 1.4 yrs (range $0.33-5.5$ yrs). The highest geometric mean concentrations after the $3 \mathrm{rd}$ dose were for serotypes $14(4.53 \mu \mathrm{g} / \mathrm{mL}, 95 \%$ CI: $2.27-6.78)$ and $19 \mathrm{~F}(4.84 \mu \mathrm{g} / \mathrm{mL}, 95 \%$ CI: $3.37-6.31)$. The lowest titres were for serotype $9 \mathrm{~V}$ following primary series $(0.96 \mu \mathrm{g} / \mathrm{mL}, 95 \% \mathrm{CI}$ : $0.64-1.28)$. In contrast to $1.5-3 \%$ of subjects after dose 2 , no subject achieved titres $<0.15 \mu \mathrm{g} / \mathrm{mL}$ after the $3 \mathrm{rd}$ dose of Prevnar ${ }^{\mathrm{TM}}$. The most pronounced boosting effects with polysaccharide vaccine were noted for serotypes 4 and 19F.

CONCLUSION: The conjugate pneumococcal vaccine was immunogenic in solid organ recipients. Three doses of the conjugate vaccine produced a more complete response than 2 doses. The polysaccharide vaccine was useful in boosting the response to low immunogenic serotypes.

\section{P16 \\ EFFICACY OF 23-VALENT POLYSACCHARIDE PNEUMOCOCCAL VACCINE (PPV) AGAINST INVASIVE PNEUMOCOCCAL INFECTION AND PNEUMONIA, ONTARIO}

A McGeer, Mount Sinai Hospital; KA Green, Mount Sinai Hospital; M Lovgren, National Centre for Streptococcus; G Tyrrell, National Centre for Streptococcus; A McGeer, Mount Sinai Hospital

INTRODUCTION: S pneumoniae is the most common cause of communityacquired pneumonia and bacteremia in adults. The National Advisory Committee on Immunization (NACI) recommends PPV for adults $>65 y$, and those aged $>2 y$ with immunocompromising conditions. We used the indirect cohort method to estimate vaccine efficacy (VE) in different adult populations.

METHODS: Population-based surveillance in metropolitan Toronto and Peel Region for invasive pneumococcal disease (IPD) from 1/1995 and for culture confirmed non-bacteremic pneumococcal pneumonia (NBPP) from 1/2002. Isolates serotyped at National Center for Streptococcus. Underlying disease categorized as immunocompromising or not. Vaccine status prior to infection obtained via medical records, patient and physician interview. VE estimated using indirect cohort method.

RESULTS: Of 3138 adult IPD episodes, 2615 occurred in persons eligible for PPV. For 2312 (82\%) isolates/serotypes were available; for 1660 of these $(73 \%)$ vaccination history was available. Overall $1453(87 \%)$ of episodes were caused by vaccine-type strains: the most common serotypes were: $14(\mathrm{~N}=274,17 \%), 3$ (160, 9.6\%), 4 (142, 8.6\%), 6B (137, 8.3\%), and $23 \mathrm{~F}(132,8.0 \%) .1246$ episodes $(75 \%)$ occurred in immunocompetent patients. VE against IPD was 50\% (95\% CL 33\%, 63\%):52\% (32\%, 67\%) for immunocompetent patients and $41 \%(-1 \%, 67 \%, \mathrm{P}=0.07)$ for immunocompromised patients. VE did not decrease with increasing age. There was no evidence of waning VE over time: VE was 54\% (32\%, 68\%) for vaccine received $<3$ y previously, $47 \%(0,60 \%)$ for vaccine received $3-6 y$ previously, and $64 \%(35 \%, 80 \%)$ for vaccine received $>6 y$ prior. There were 341 episodes of NBPP from 2002 to 2004; 295 in adults eligible for PPV. Of these, 250 (85\%) had isolates/serotypes available, and 165 of these $(66 \%)$ had vaccination history available. 122 (74\% of episodes were caused by vaccine-type strains; the most common serotypes were: $3(\mathrm{~N}=27$, $16 \%), 19 \mathrm{~F}(25,15 \%), 6 \mathrm{~B}(16,9.7 \%)$ and $6 \mathrm{~A}(13,7.9 \%)$. Estimated VE against NBPP was $16 \%$ (95\% CI $-29 \%, 58 \%)$.

CONCLUSION: PPV is clearly efficacious against IPD in eligible adult recipients, and likely provides protection even for immunocompromised patients. Re-vaccination at $<10$ years does not appear warranted. We found no evidence for PPV protection against NBPP, which may be in part due to serotype differences between NBPP and IPD. 


\section{POSTER PRESENTATIONS Public Health Research}

\section{P17}

\section{POUR UNE VACCINATION CONTRE L'INFLUENZA ET LE PNEUMOCOQUE PLUS PERFORMANTE DANS LES GROUPES DE MÉDECINE DE FAMILLE}

J St-Cerny, Direction de santé publique; M Guay, Université de Sherbrooke, Centre de recherche Hôpital Charles LeM; D Roberge, Université de Sherbrooke, Centre de recherche Hôpital Charles Le CONTEXTE ET OBJECTIFS: Afin d'améliorer la performance des programmes de vaccination contre l'influenza (VI) et le pneumocoque (VP), les groupes de médecine de famille (GMF) semblent prometteurs. Ils ont plusieurs attributs intéressants pour ces deux programmes. Notre étude vise à apprécier la faisabilité d'un modèle d'organisation des services de VI et VP en GMF et à identifier les conditions nécessaires à son implantation. MÉTHODE: Étude descriptive réalisée en 2006 auprès des responsables de la vaccination des 19 GMF de la Montérégie au Québec. 1) Entrevues téléphoniques: variables à l'étude: pratiques actuelles d'immunisation selon un modèle de référence (créé selon les pratiques visant à améliorer l'offre, la demande ou l'accessibilité à la vaccination); analyses: écarts par rapport au modèle. 2) Entrevues face à face: variables à l'étude: opinion face aux stratégies du modèle et aux écarts observés, suggestions pour améliorer la VI et la VP en GMF; analyses de contenu.

RÉSULTATS: Volet 1 (taux de participation 84\%). Les pratiques vaccinales des GMF sont variées. Pour le VI, 70\% des GMF font de la promotion, $48 \%$ font des rappels téléphoniques, tous vaccinent lors de toutes visites et $91 \%$ font un rappel au vaccinateur. Peu de VP n'est faite en dehors de la campagne VI. Seulement 17\% des GMF n'utilisent pas les quatre catégories de stratégies du modèle de référence. Volet 2 (taux de participation 53\%). Tous les médecins considèrent que c'est leur rôle d'offrir la vaccination au sein du GMF. Le rappel est une stratégie favorisée mais à condition que les ressources humaines et informationnelles soient disponibles. Connaître les $\mathrm{CV}$ obtenues et donner une rétroaction à chaque médecin seraient aussi efficaces. L'absence de rémunération pour la vaccination est dissuasive. On reconnaît devoir faire mieux pour la VP. DISCUSSION: Les médecins des GMF sont motivés et intéressés par la vaccination. Un ajout de ressources, par ex. un système d'information performant, est considéré essentiel à une meilleure efficacité des services de vaccination en GMF.

\section{P18 \\ COÛTS ET EFFICACITÉ DU PROGRAMME DE VACCINATION CONTRE L'INFLUENZA AU QUÉBEC}

M Guay, Université de Sherbrooke; M Blackburn, Direction de santé publique (DSP) Montérégie, Longueuil, Québec; A Pelletier, Institut national de santé publique du Québec, Montréal, Québec; A Tremblay, DSP Montérégie; C St-Hilaire, AÉTMIS, Montréal, Québec; AM Clouatre, DSP Montérégie, Longueuil, Québec; L Rousseau, DSP Montréal, Montréal, Québec et U de Montréal; $M$ Landry, Ministère de la santé et des services sociaux (MSSS) Montréal, Québec; P Clément, Institut national de santé publique du Québec, Montréal, Québec; M Dionne, Institut national de santé publique du Québec, Québec, Québec; D St-Amandaqes, Montréal, Québec

OBJECTIFS: 1) Évaluer les coûts et l'efficacité du programme québécois d'immunisation contre l'influenza. 2) Comparer les coûts selon le lieu de vaccination (Centre local de services communautaires (CLSC) ou clinique médicale $(\mathrm{CM}))$.

MÉTHODE: Devis quantitatif et qualitatif selon une perspective sociétale (MSSS, DSP, CLSC, médecins vaccinateurs et adultes de 60-64 ans considérés). L'approche de micro-costing a utilisé 15 sources de données (collecte réalisée en 2005-2006). L'évaluation des coûts porte sur un échantillon de dix territoires de CLSC sélectionnés selon leurs caractéristiques sociodémographiques et celles des services d'immunisation. Des estimations selon la perspective des acteurs sont établies pour des coûts totaux annuels (CTA) et des coûts unitaires par dose (CPD). Selon la perspective sociétale, un ratio coût-efficacité (efficacité évaluée par proxy: couverture vaccinale contre l'influenza) et une analyse de minimisation des CPD sont présentés.

RÉSULTATS: Les données préliminaires avant validation montrent que le CTA assumé par le MSSS est de 8 millions \$. Les CTA assumés par les DSP varient de 22752 \$ à 291361 \$. Le CPD assumé par les CLSC est de 11 \$ et celui assumé en CM est de 17 \$. Le CPD assumé par les adultes est de 8 \$. La couverture vaccinale des 60-64 ans s'élève à $48 \%$. Le ratio coûtefficacité est de $94 \$$ par dose pour chaque point de pourcentage de couverture vaccinale obtenu. Selon la perspective sociétale, en retirant les déboursés des vaccins, le CPD en CLSC est de 26 \$ et de 27 \$ en CM. Le coût de la vaccination en CLSC est légèrement plus faible dans l'analyse de minimisation des coûts et l'analyse de sensibilité.

CONCLUSION: Outre les déboursés pour les vaccins, la vaccination contre l'influenza génère des coûts non négligeables. Les résultats de l'étude pourront être utilisés pour assurer l'adéquation du financement des services aux coûts réels.

\section{P19}

\section{RETARDS DE VACCINATION DES NOURRISSONS - QUI EN EST RESPONSABLE?}

M Guay, Université de Sherbrooke; V Fradette, Université de Sherbrooke, Longueuil, Québec; C Francoeur, Université de Sherbrooke, Longueuil, Québec; F Pichette, Université de Sherbrooke, Longueuil, Québec; K Toulouse, Université de Sherbrooke, Longueuil, Québec; J Dufort, Université de Sherbrooke, Longueuil, Québec; M Guay, Université de Sherbrooke, Longueuil, Québec et DSP Montérégie

INTRODUCTION: Avec l'ajout récent de plusieurs vaccins au calendrier, des retards sont constatés chez certains nourrissons. L'étude vise à décrire les causes des retards de vaccination des enfants et les perceptions des parents d'enfants vaccinés à temps (PEVT) et des parents d'enfants vaccinés en retard (PER).

MÉTHODE: Enquête téléphonique réalisée en mars 2006 auprès de 116 parents d'enfants de 2 à 6 mois vaccinés dans une des 106 cliniques médicales de la Montérégie au Québec. Les variables à l'étude inspirées du Health Belief Model étaient entre autres: la perception des bénéfices et des risques de la vaccination, la méthode de prise de rendez-vous, la cause du retard et les caractéristiques sociodémographiques. Des analyses descriptives sont faites et les PEVT et PER sont comparés.

RÉSULTATS: Les caractéristiques sociodémographiques des PEVT et PER sont semblables. La majorité des parents (>75\%) perçoivent les bénéfices de la vaccination et sont d'avis que les avantages surpassent les inconvénients, il n'y a pas de différence entre les PEVT et PER. Pour $41 \%$ des PER, le retard a été causé par un problème personnel (ex. maladie de l'enfant, oubli) alors que pour les autres, le délai trop long pour obtenir un rendez-vous a été incriminé. Les PEVT ont pris leur rendez-vous lors d'une visite précédente ( $66 \%$ vs $40 \%$; p=0,02) et les PER ont fait face à des délais plus longs entre le moment de la prise de rendez-vous et la date effective de la vaccination ( 57 jours vs 34 jours; $\mathrm{p}<0,05$ ).

CONCLUSION: Bien que les retards de vaccination puissent être reliés à des causes personnelles des parents, la majorité des retards est attribuable à un problème de l'organisation des services. Une amélioration dans la méthode de prise de rendez-vous et de la disponibilité de rendez-vous de vaccination est souhaitable.

\section{P20}

\section{IMPACT DE L'AJOUT DES NOUVEAUX VACCINS SUR LE RESPECT DU CALENDRIER}

A Hamid, Université de Sherbrooke; M Guay, Université de Sherbrooke, Longueuil, Québec; J Lemaire, Université de Sherbrooke, Longueuil, Québec; AM Clouâtre, Direction de santé publique de la Montérégie, Longueuil, Québec

CONTEXTE ET OBJECTIFS: Plusieurs vaccins ont été récemment ajoutés au calendrier des nourrissons. Cet ajout pourrait se traduire par le non respect du calendrier, en raison notamment des multiples injections 
lors d'une visite. La présente étude vise à explorer l'impact de l'ajout des vaccins sur l'observance au calendrier de vaccination.

MÉTHODE: Étude transversale par questionnaire postal auto-administré (janvier 2006) auprès des parents de deux échantillons d'enfants tirés du Fichier des naissances de la Montérégie (Québec) : cohorte 1 - enfants nés entre avril 2002 et mai 2003 (ancien calendrier); cohorte 2 - enfants nés entre avril 2004 et mai 2005 (nouveau calendrier). Les variables à l'étude sont: vaccins reçus, dates de vaccination, variables sociodémographiques et opinion sur les injections multiples. Après avoir complété les données vaccinales auprès des vaccinateurs, des analyses descriptives, détermination du statut vaccinal et comparaisons entre cohortes ont été réalisées.

RÉSULTATS: Le taux de réponse fut de $56 \%$ (1 457/2 615). La majorité (85\%) des enfants de la cohorte 1 ont reçu tous les vaccins recommandés pour l'âge. Aucune association n'a été trouvée entre les caractéristiques sociodémographiques et le statut vaccinal. L'analyse de l'observance du calendrier par approche de survie de Kaplan Meier montre un retard de réception des vaccins dans la cohorte 2 comparée à la cohorte 1 dès la deuxième visite (Nombre de jours médians de retard : 3 jours sur le 2è $(\mathrm{p}<0,016), 4$ jours sur le 3è $(\mathrm{p}<0,001), 11$ jours sur le 4è DCaT-Polio-Hib $(\mathrm{p}<0,001) ; 32$ jours sur le 1er RRO $(\mathrm{p}<0,001)$ et 7 jours sur le 2è $\mathrm{RRO}$ $(\mathrm{p}<0,001))$. Plusieurs parents $(51 \%)$ préfèrent une à deux injections lors d'une même visite pour leur enfant.

DISCUSSION/CONCLUSION: Des actions devraient être faites afin de corriger les délais de vaccination constatés et de favoriser une meilleure observance au calendrier de vaccination.

\section{P21}

\section{COUVERTURE VACCINALE CONTRE L'INFLUENZA EN 2005 DES ENFANTS QUÉBÉCOIS ÂGÉS ENTRE 6 ET 23 MOIS ET DE LEURS CONTACTS DOMICILIAIRES}

N Boulianne, INSPQ; G Guay, Institut national de santé publique du Québec; B Duval, Institut national de santé publique du Québec; L Valiquette, Direction de santé publique de Montréal; M Ouakki, Centre de recherche du CHUQ; G De Serres, Institut national de santé publique du Québec

INTRODUCTION: En 2004, le Comité consultatif national sur l'immunisation (CCNI) recommandait la vaccination contre l'influenza des enfants âgés entre 6 mois et 23 mois et de leurs contacts domiciliaires. Deux ans après l'instauration de ce programme au Québec, il est important de connaître la couverture vaccinale dans ce groupe et d'analyser certains déterminants de la vaccination.

MÉTHODOLOGIE: Il s'agit d'une étude descriptive transversale réalisée auprès d'un échantillon aléatoire de 500 enfants nés entre le 1e décembre 2003 et le 31 mai 2005 obtenu auprès de la Régie de l'assurance maladie du Québec. Un questionnaire postal auto-administré a été envoyé en avril 2006. Deux relances postales et une téléphonique ont été faites auprès des non répondants. Un enfant a été considéré complètement vacciné s'il a reçu au moins 2 doses d'un vaccin influenza dont au moins une après le 15 octobre 2005.

RÉSULTATS: Un total de 366 questionnaires sur 490 éligibles ont été retournés pour un taux de réponse de $75 \%$. La couverture vaccinale 2 doses contre l'influenza est de 34,2\% ( IC 95\% : 29,3\% - 39,3\%) et s'élève à $41,3 \%$ ( IC $95 \%: 36,2 \%-46,5 \%$ ) pour une seule dose en 2005 . Les contacts domiciliaires des enfants visés par l'enquête ont été vaccinés dans une proportion de $30 \%$ (34\% chez les moins de 18 ans et $28 \%$ chez les 18 ans et plus). En analyse bivariée, il n'a pas été possible d'identifier de facteurs socio-démographiques associés significativement à la vaccination influenza. CONCLUSION: Seulement le tiers des enfants et contacts visés ont été adéquatement vaccinés contre l'influenza en 2005 au Québec. Des stratégies devront alors être développées pour augmenter significativement cette proportion. Il est important de continuer à suivre l'évolution de la couverture vaccinale pour évaluer l'impact des interventions visant à mieux rejoindre cette population.

\section{P22}

\section{THE 2005 NATIONAL GOALS AND RECOMMENDATIONS CONSENSUS CONFERENCE}

A-M Frescura, Public Health Agency of Canada (PHAC); C Navarro, PHAC; L Belzak, PHAC

INTRODUCTION: The 2005 National Consensus Conference for Vaccine-Preventable Diseases was the first in a series of consensus conferences that will review disease reduction goals and immunization coverage targets for all vaccine preventable diseases. The purpose was to review and assess the current status of existing disease reduction goals and immunization coverage targets for varicella, invasive meningococcal disease, invasive pneumococcal disease, pertussis, rubella and influenza.

METHODS: Working groups reviewed current evidence, identified key issues and revised or developed disease reduction and immunization coverage goals and recommendations. Conference participants voted in plenary on proposed recommendations, with $75 \%$ agreement or agreement with reservation required for consensus on a proposed recommendation. Jurisdictions were surveyed after the conference to assess the feasibility, measurability and level of priority of the newly developed 2005 national goals and recommendations.

RESULTS: Consensus was reached on 52 updated recommendations for all six diseases. Conference delegates voted overwhelmingly in favour of adopting the Pan-American Health Organization regional goal to eliminate indigenously transmitted cases of rubella and congenital rubella syndrome from Canada by 2010. Goals and recommendations were developed or revised to account for the introduction of new meningococcal, pneumococcal and pertussis vaccines. For influenza, it was agreed by consensus to adopt previous (2001) national immunization coverage targets and to postpone the development of disease reduction recommendations for a future conference. Inadequate surveillance data and lack of resources available to develop new data collection tools and monitoring systems were identified as potential barriers for attaining or adequately measuring progress towards goals and recommendations.

CONCLUSIONS: The Canadian Immunization Committee has recently approved national adoption and publication of the 2005 goals and recommendations, with the intention of measuring progress towards targets annually. Efforts need to be directed towards improving immunization programs and surveillance and developing the infrastructure needed for measuring immunization coverage.

\section{P23}

\section{MEASURING SUCCESS OF THE NATIONAL IMMUNIZATION STRATEGY}

A-M Frescura, Public Health Agency of Canada (PHAC); L Belzak, PHAC; A Frescura, PHAC; M Farhangmehr, PHAC

INTRODUCTION: The National Immunization Strategy (NIS), approved and funded in 2004, was designed to address challenges to planning and implementing safe, efficient, and effective immunization programs in Canada. A comprehensive evaluation framework has been developed to monitor the progress and measure the success of the NIS. The evaluation process will be implemented in two phases. Phase I of the evaluation is a formative (interim) evaluation that will monitoring the implementation of NIS activities and outputs achieved.

METHODOLOGY: The evaluation framework identifies nine activities to be evaluated. A performance measurement strategy outlines the indicators and data sources for each activity's outputs and outcomes. Document review, and surveys measure the indicators for the outputs. The framework contains evaluation questions, their indicators and data sources. The evaluation questions focus on four main areas; relevance; impact and effect; design and delivery; and cost-effectiveness. Key informant interviews and document reviews are being used to measure the indicators.

RESULTS: The successes of the NIS can be grouped in three major theme areas; implementation and accountability; collaboration and leadership; and leveraging of resources. All nine NIS activities have been implemented to date. The majority of activities have representational leadership in the form of working groups. To ensure accountability, each group has a terms of reference, produce records of decisions and oversee yearly workplans. 
Leverage is one of the most important and high profile NIS accomplishments to date. Increased collaboration, number of partnerships have been observed on as a result of the NIS, immunization program have been introduced and expanded with funding from the NIS other resources.

CONCLUSIONS: The current structure of accountability, collaboration has contributed to the success of the NIS. The major challenges identified in achieving the outcomes are human resource and infrastructure to support the activity expansion.

\section{P24}

RESULTS FROM THE 2004 \& 2006 NATIONAL CHILDHOOD IMMUNIZATION COVERAGE SURVEYS A-M Frescura, Public Health Agency of Canada (PHAC); J Njihia, PHAC; L Belzak, PHAC

INTRODUCTION: National Immunization Coverage Surveys (NICS) are implemented routinely every 2 years by the Public Health Agency of Canada in order to assess national immunization coverage rates for routine childhood immunizations. The purpose is to assess up-to-date coverage rates (correct number of vaccine doses received according to NACI recommended scheduled) and on-time coverage rates (up-to-date with timeliness of doses adhered to), changes in knowledge attitudes and beliefs $(\mathrm{KAB})$ and to monitor progress towards national immunization coverage goals.

METHODS: The 2004 Childhood NICS employed a telephone survey of households with children to estimate routinely recommended childhood immunization coverage rates of children by their 2 nd, 7 th and 17 th birthdays. Changes made since the 2002 survey include the addition of the 17 year age group, the addition of four new publicly funded vaccines and the KAB section was expanded. The 2006 NICS employs similar methodology and preliminary results are expected by late October.

RESULTS: Up-to-date coverage rates for 1 dose of MMR (measles, mumps, and rubella) by 2 years was unchanged from 2002 at $94 \%, 78 \%$ for a second dose by 7 years compared to $74 \%$ in 2002 and $62 \%$ for 2 doses by 17 years. Coverage rates for diphtheria, pertussis, tetanus, polio and haemophilus influenzae Type B vaccine were unchanged or slightly higher than in 2002 at $78 \%, 74 \%, 73 \%, 89 \%$ and $70 \%$ respectively. Coverage rates for the booster dose of diphtheria and tetanus at 17 yrs were low at $47 \%$ and $44 \%$ respectively. On-time coverage rates were highest for MMR at 2 years of age $(50 \%)$.

CONCLUSION: Up-to-date coverage status has improved across all antigens for 2 and 7 year olds. On-time coverage was significantly lower than up-to-date status for all antigens, prompting the need for more timely dose administration for improved public health protection.

\section{P25}

\section{VALIDATION OF CHILDREN'S IMMUNIZATION STATUS REPORTED BY PARENTS FROM HAND-HELD IMMUNIZATION CARDS}

A-M Frescura, Public Health Agency of Canada; J Njihia, Public Health Agency of Canada; A Frescura, Public Health Agency of Canada; L Belzak, Public Health Agency of Canada

INTRODUCTION: National Immunization Coverage Surveys (NICS) are routinely conducted by the Public Health Agency of Canada (PHAC), to monitor national routine immunization coverage levels of Canadian children. Inaccuracies such as significantly different coverage estimates for antigens routinely administered as one single vaccine were noticed in the 2002 NICS. This calls into question parent's ability to recall their child's immunizations, their ability to read immunization records or the quality of the immunization records itself. A validation component was added to 2004 NICS to evaluate the accuracy of parental reporting from hand-held immunization cards.

METHODS: With parents consent, immunization records were retrieved from the children's health care providers. By considering the providers' records as the gold standard, the validity of up-to-date (UTD) immunization status reported by parents was assessed by calculating the percentage of agreement, positive predictive value (PPV) and negative predictive value (NPV).
RESULTS: A total of 205 children were included in validation analysis. Validation coverage rates for 2 year olds were higher than NICS results for all antigens, with the largest difference at $12.1 \%$ for diphtheria, tetanus and Hib. Within validation results, differences of 5-8\% (diphtheria, pertussis, tetanus, polio) remained among antigens which are normally administered together. Some major obstacles included the inability to link some retrieved records to the original survey database and the small sample size of the 7 and $17 \mathrm{yr}$ age groups, which prevented any conclusion to be drawn for these groups.

CONCLUSIONS: Parental reporting from hand-held immunization cards generally underestimates coverage for 2 year olds. Based on these findings, future consideration include modifying the NICS to collect information by vaccine as well as by antigen and to conduct a more extensive validation survey including all survey participants, not only a sub-set.

\section{P26}

\section{MEASURING ADULT INFLUENZA AND PNEUMOCOCCAL IMMUNIZATIONS, CANADA 2006}

A-M Frescura, Public Health Agency of Canada; A Frescura, Public Health Agency of Canada; L Belzak, Public Health Agency of Canada; M Farhangmehr, Public Health Agency of Canada

INTRODUCTION: In 2006 the Public Health Agency of Canada (PHAC) sponsored the Adult National Immunization Coverage Survey (NICS). The primary objective of the survey was to assess coverage rates for influenza and pneumococcal adult vaccines, and to compare the coverage rates obtained with national targets. Immunization coverage targets recommended by the National Advisory Committee on Immunization's (NACI) for non-institutionalized adults $\geq 65$ years and adults with high risk medical conditions are $70 \%$ for influenza and $80 \%$ for pneumococcal. The secondary objective of the NICS was to assess knowledge, attitudes and behaviors towards immunization, missed opportunities of immunization and circumstances around immunization.

METHODS: The 2006 Adults NICS used a telephone survey to assess coverage for 4429 French or English speaking non-institutionalized Canadian adults. Specific target groups included: seniors $\geq 65$ yrs, health care workers $(\mathrm{HCW}) /$ volunteers, adults $18-64$ years with chronic medical conditions and adults 18-64 years with no chronic medical conditions. The survey collected immunization information on pneumococcal, influenza and hepatitis B, hepatitis A, tetanus, pertussis and varicella vaccines. For influenza, pneumococcal and hepatitis B, the results were compared with results obtained from the 2001 Adult NICS.

RESULTS: Compared to results from 2001, influenza coverage increased by $2.4 \%$ to $40.5 \%$ among adults with chronic medical conditions, and by $12.7 \%$ to $63.8 \%$ among HCW. There was no change among seniors (69.1\% compared to $69.7 \%$ ). Compared to results from 2001, pneumococcal immunization coverage dropped by $5.7 \%$ to $36.5 \%$ among seniors, and by $3.5 \%$ to $11.9 \%$ among adults with chronic medical conditions.

CONCLUSIONS: Although Canada has met its target coverage rate for influenza immunizations in seniors, it remains short of these goals for adults with chronic medical conditions. Canada continues to drop further from it target for pneumococcal coverage among at risk populations. Hence, efforts need to be put in place to improve pneumococcal coverage.

\section{P27}

ADOLESCENT HEPATITIS B PROGRAMS IN CANADA: THEIR SUCCESS AND APPLICABILITY TOWARDS HUMAN PAPILLOMAVIRUS VACCINE PROGRAM IMPLEMENTATION A-M Frescura, Public Health Agency of Canada (PHAC); L Belzak, PHAC; S Deeks, PHAC; M Farhengmehr, PHAC; L Schiedel, Ministry of Health and Long Term Care, Ontario; E Sartison, Disease Control and Prevention Branch, Government of Alberta; A Coombs, Government of Nova Scotia

INTRODUCTION: The quadrivalent human papillomavirus (HPV) vaccine, Gardasil ${ }^{\mathrm{TM}}$ was approved for use in Canada on July 10, 2006, and recommendations are currently being developed for this new vaccine. School-based hepatitis B (Hep B) programs in Canada have been very successful and may provide a model for HPV vaccine delivery to adolescents. 
Information on provincial and territorial school-based Hep B immunization programs was collected to assist with decision-making.

METHODS: A brief survey was distributed to provincial and territorial representatives of the Canadian Nursing Coalition for Immunization (CNCI) to collect information on current and past school-based Hep B programs. Respondents were asked to report coverage rates and the method of data collection used to assess coverage for each year.

RESULTS: All provinces and territories currently have or have had school-based immunization programs. Three provinces responded to this survey: Alberta, Nova Scotia and Ontario. Alberta delivers school-based Hep B program to Grade 5 children, but does not measure coverage for this program. Ontario has a grade 7 school-based program in place and has averaged a coverage rate of $86 \%$ (range $81 \%-94 \%$ ) for three doses of Hep B from 1994 until 2005. In Nova Scotia, a school-based Hep B program is delivered to children in Grade 4 and coverage rate averaged $97 \%$ from 2000 to $2005 / 06$. Based on these results, provincial coverage rates far surpass national rates (60\% in 2004 for 3 doses of Hep B by 17 years).

CONCLUSION: Evidence from this survey suggests that school-based Hep B programs are successful in a select number of provinces and territories in Canada and should be considered as a viable option for HPV vaccine delivery. More extensive responses to this survey will provide further evidence for implementing school-based programs for HPV vaccine and will be included in the final presentation.

\section{P28}

\section{EVALUATION OF A PILOT PROGRAM OF COMMUNITY PHARMACY-BASED INFLUENZA IMMUNIZATION CLINICS} $S$ Bowles, Division of Geriatric Medicine-Dalhousie University; RA Strang, CDHA; E Wissemann, Dalhousie University; EK Black, Dalhousie University

INTRODUCTION: Annual influenza vaccination remains the most effective strategy for reducing influenza-related complications, but immunization rates remain below national targets in many jurisdictions. Community-pharmacy based clinics have the potential to enhance immunization rates in some populations. We report our experience with a pilot program of community pharmacy-based clinics in one Nova Scotia District Health Authority.

METHODS: The program was implemented in the fall of 2003, with a total of 42 clinics held within the Capital District Health Authority (CDHA). CDHA Public Health Services supplied vaccine for all clinics and nursing staff/supplies at 10 clinics. At the remaining 32, pharmacies contracted a private nursing agency and covered the cost of supplies. Each pharmacy was responsible for promotion of the clinics and referral of highrisk patients, identified by screening medication profiles. Registered nursing staff administered all immunizations. All clinic attendees completed a feedback survey and were encouraged to provide additional comments.

RESULTS: A total of 2828 adult patients were immunized at 42 clinics (average 67/clinic). 20\% reported not having been previously immunized. $50 \%$ were high-risk individuals ( $790>65$ years, $598<65$ years), $26 \%$ household contacts, $12 \%$ health care professionals/first responders and $12 \%$ non-high risk persons who chose to pay out of pocket. $80 \%$ of clinic attendees reported the pharmacy-based clinics were their preferred site for receiving immunization. The most frequently cited reasons were convenience, less waiting time, not having to make an appointment, and easier parking. Opportunities for improvement included increasing clinic hours and immunizing in an area of the pharmacy that provided more privacy. There were no complaints from local physicians about the clinics and many referred patients to them.

CONCLUSIONS: Community pharmacy-based influenza immunization clinics may provide an additional model to enhance vaccination rates among some high-risk individuals.

\section{P29}

ASSESSING KNOWLEDGE, ATTITUDES, AND BEHAVIOURS REGARDING VACCINES AMONG PUBLIC HEALTH NURSES IN NOVA SCOTIA

L Pelly, Canadian Centre for Vaccinology; BA Halperin, Canadian

Centre for Vaccinology, Halifax; J MacPhee, Nova Scotia

Department of Health; SA McNeil, Canadian Centre for

Vaccinology, Halifax

INTRODUCTION: Public health nurses are an important source of immunization information for the public whether or not they provide immunization services. Understanding immunization-related knowledge, attitudes, and behaviours of Nova Scotia (NS) Public Health Nurses will allow the development of targeted, evidence-based Continuing Professional Development initiatives designed to enhance the role of this group in ensuring the success of public immunization programs.

METHODS: The 98-item self-administered survey instrument was developed at the Canadian Centre for Vaccinology, Halifax and validated through focus groups, content validity, and test-retest reliability. It was distributed to all 171 NS Public Health Nurses via Regional Communicable Disease Managers.

RESULTS: The response rate was $45.6 \% ; 82.1 \%$ of respondents were employed full-time with NS Public Health Services. As expected from this population, knowledge scores were high with a mean score of 18.1 out of 21. Interestingly, despite the population being surveyed, some individuals were unsure or thought there was a link between vaccines and conditions such as autism, multiple sclerosis, and sudden infant death syndrome. The theme, however, was one of potential missed opportunities. $43.4 \%$ of respondents indicated that they would withhold vaccinations in cases of mild illness with fever and $26.6 \%$ thought that pneumococcal vaccine was contraindicated for asplenic patients. Finally, only $43.6 \%$ indicated that they routinely recommend vaccines not yet publicly-funded.

CONCLUSIONS: Public health nurses are a group of health professionals that are educated and experienced in regards to immunizations and are an important information resource for the public. Although public health nurses in NS are generally knowledgeable and have positive attitudes regarding vaccines, there remains potential for missed opportunities to immunize. Continuing Professional Development initiatives should focus on true indications/contraindications to minimize missed opportunities and arm public health nurses with accurate information regarding potential associations between vaccines and acute and chronic medical problems.

\section{P30 \\ INVESTIGATION OF PHYSICIAN IMMUNIZATION KNOWLEDGE, ATTITUDES AND PRACTICE IN VANCOUVER COASTAL HEALTH, NORTH SHORE: A QUALITATIVE APPROACH}

L Buchanan, Vancouver Coastal Health; D McKellar, Vancouver Coastal Health; M Guenther, Vancouver Coastal Health; B O'Connor, Vancouver Coastal Health; J McCaffrey, Vancouver Coastal Health; J Addison, Vancouver Coastal Health INTRODUCTION: In the North Shore area of Vancouver Coastal Health, physicians provide $75 \%$ of childhood immunizations and are required to provide immunization statistics to Public Health (PH). Introduction of new vaccines into the routine provincial schedule has decreased data reporting and increased reports of vaccine-related errors. This study's purpose was to identify immunization challenges for physicians, including the perception of their role in reporting. The second objective was to identify how physicians access immunization information and best methods for ongoing education and resources to support practice. METHODS: $10 \%$ of immunizing physicians were recruited, using strict inclusion criteria, by an independent health marketing research firm. Participants were interviewed, one on one, regarding vaccine storage, handling, recording, reporting, patient safety, counselling, attitudes and professional development.

RESULTS: Participants stated they have growing concerns about increasingly complex immunization programs. Lack of knowledge on handling and storage of biological products, including epinephrine dosing was evident. 
Participants identified office staff as being responsible for day to day management of immunization practice. Participants stated that reporting data to $\mathrm{PH}$ was time consuming and unnecessary. The local PH Newsletter appears to be the main reference source for information and updates. Participants were unfamiliar with national and provincial expert immunization committees and their recommendations. Participants have few resources for parents with questions.

CONCLUSIONS: Physicians value immunization but face many challenges. Lack of access to up-to-date resources for themselves, staff and for patient counselling was evident as was understanding the need for reporting. Findings indicate improved communication from $\mathrm{PH}$ is required to keep physicians engaged in childhood immunizations and data reporting. Findings also indicate educational information and professional development for physicians and staff regarding handling, storage and administration of vaccines is needed along with materials for patient counselling. Results from this study have led to the development of immunization education and reporting resources for physicians and staff.

\section{P31}

PREDICTING THE IMPACT OF A VACCINE TARGETING HUMAN PAPILLOMAVIRUS 16/18 ON CERVICAL CANCER INCIDENCE AND MORTALITY IN CANADA: A MODEL BASED ANALYSIS

A Anonychuk, GlaxoSmithKline Canada; MT Do, GlaxoSmithKline Canada, Toronto; BZ Pham, Health Policy \& Management, University of Toronto; GM Van Kriekinge, GlaxoSmithKline Biologicals, Rixensart, Belgium; BA Standaert, GlaxoSmithKline Biologicals, Rixensart, Belgium

INTRODUCTION: The reality of an HPV vaccination program in Canada is fast approaching, though the long term impact of vaccination in Canada is unclear. A decision-analytic Monte-Carlo simulation model was used to explore the population level impact of a vaccine targeting HPV $16 / 18$ on incidence of invasive cervical cancer (ICC), related mortality and life-years gained in Canada

METHODS: The analysis was based on ICC incidence $(9.5 / 100,000$ women) and mortality (2.8/100,000 women) data from the Canadian Cancer Registry (1990-2001), population data (Census 2001), and HPV$16 / 18$ prevalence in cancer cases for Canada ( $47 \%$ and $23 \%$, respectively). The base-case analysis is for 11 -year old females $(n=200,333)$, vaccinated and followed over lifetime with $95 \%$ vaccine efficacy, $100 \%$ coverage, time to progression to ICC 20 years, and minimum age at infection 13 years. Sensitivity analyses were performed on these values. Impact of vaccination at ages 11, 15, 20 and 25 were estimated nationally and for each province. RESULTS: The model was validated against observed ICC incidence data in Canada. In the base case analysis, vaccination at age 11 reduced ICC cases by $66 \%$ (from 1,500 to 505 cases) and related mortality by $66 \%$ (from 468 to 157 deaths). Results were similar for vaccination at age 15 . The reduction in ICC cases and mortality for vaccination at age 25 was $40 \%$ and $53 \%$, respectively. Total life-years gained for the cohort was 7,751 years. Similar reductions were observed for all provinces, especially provinces with high ICC incidence. The results were sensitive to variation in prevalence of HPV 16/18 among cancer cases, coverage, and age of vaccination.

CONCLUSION: In Canada, vaccinating females with a vaccine targeting HPV 16/18 would result in substantial reduction in ICC cases and related mortality. This does not take into account additional potential benefits of the vaccine with cross-protection against other oncogenic HPV types as demonstrated by recent findings.

\section{P32}

COMMUNITY NURSING AND IMMUNIZATION PROMOTION; WHERE ARE WE NOW AND WHERE DO WE NEED TO GO?

T Morgana, Vancouver Coastal Health

INTRODUCTION: Community Health Nurses (CHNs) who provide healthcare to families and their children in the community are key stakeholders in immunization promotion. In October 2005, Vancouver Coastal Health (VCH) assessed how CHNs can be supported to improve communications and service delivery. Key elements to the success of immunization promotion that were explored with the CHNs included: risk communication skills, use and effectiveness of resources, education needs, information distribution and infrastructure.

METHODS: A convenience sample of $226 \mathrm{VCH} C H N$ s received a 20-minute, self-administered questionnaire by mail, consisting of both qualitative and quantitative questions.

RESULTS: One hundred CHNs responded. Only 29\% of CHNs are "very comfortable" addressing difficult safety topics with parents, and reported current educational materials lack effectiveness to answer common questions. There is no continuing education standard, and over $40 \%$ of $\mathrm{CHNs}$ are committing five or more hours per month to self-education. Awareness and use of a variety of good print and electronic resources is low. Over $45 \%$ of nurses "never" ask clients if they wish to receive information by email. Finally, over ten themes were developed providing insight into information dissemination and infrastructure issues.

CONCLUSIONS: A multi-faceted, strategic, immunization promotion plan will incorporate the identified needs of this survey. A standardized continuing education program including risk communication skill building, computer skill building, and familiarity of educational resources is needed. Updated targeted educational materials using plain language principals and graphic design are essential. A redesign of information dissemination should be considered, and an advocacy plan for infrastructure investment should also be developed. Some needs can be addressed in the short term using current resources, while other gaps will require significant investment and may be better approached through collaborative efforts at the provincial and national level.

\section{P33 \\ DETERMINANTS FOR IMMUNIZATION COVERAGE BY AGE 2 FOR FIVE RECOMMENDED VACCINES IN THE CAPITAL HEALTH REGION (EDMONTON)}

J Zhang, University of Alberta; A Ohinmaa, University of Alberta, School of Public Health; Institute of Health Economics; T Nguyen, Alberta Health and Wellness; L Mashinter, Capital Health;

A Brown-Ogrodnick, Capital Health; A Hanrahan, Capital Health; J Loewen, Capital Health; W Vaudry, University of Alberta,

Department of Pediatrics; P Jacobs, University of Alberta, School of Public Health; Institute of Health Economics

INTRODUCTION: The Canadian goal for the complete routine childhood immunization coverage rate for children up to 24 months of age is set to be at least $97 \%$. This study analyzes the impact of background variables on the childhood immunization coverage rates in the Capital Health $(\mathrm{CH})$ region in Alberta.

METHODS: The study population consisted of all registered children in $\mathrm{CH}$ who were born between 1 July and 31 December, $2002(\mathrm{~N}=4,988)$. We followed each child from birth until their second birthday. Immunization status for five recommended vaccines was determined according to the Alberta childhood immunization schedule. We performed logistic regression, regressing children's immunization status on vital statistics, including mothers' marital status, feeding methods, number of siblings, and children's socioeconomic background. The data sources were from the $\mathrm{CH}$ immunization registry and Alberta Wellnet Electronic Health Record database that includes comprehensive demographic and socioeconomic information. In the logistic regression, full coverage for each vaccine was compared to partial coverage (vaccine program started but not completed by age two) and no coverage (program not started).

RESULTS: The range of complete coverage rates in the study varied from the lowest $83.8 \%$ (Pneumococcal Conjugate) to the highest $94.2 \%$ (Meningococcal Conjugate). Variables on who was delivered by a midwife and number of siblings are negatively correlated with immunization rates. The coverage rates of the babies in income support programs (native and/or non-native) were significantly lower than those not in the programs. The babies who were second born in the family with a mother older than 30 years of age had significantly higher immunization levels.

CONCLUSIONS: There are some key determinants that impact on overall coverage rates but there are also several vaccine-specific determinants. 


\section{P34}

READ THE LABEL - PREVENTABLE VACCINE ADMINISTRATION ERRORS

J Shindman, sanofi pasteur; N Rozon; HA Rohacek

INTRODUCTION: Telephone call from poison-control centre: "A doctor combined PENTACEL ${ }^{\circledR}$, Prevnar ${ }^{\circledR}$, Neisvac-C Vaccine and RecombivaxHB ${ }^{\circledR}$ in one syringe and administered it to a child. What do you recommend?" In July 2004, a summary of incidents in which tetanus toxoid-containing vaccines (TTCV) were given intradermally instead of tuberculin purified protein derivative (PPD) was published in the Morbidity and Mortality Weekly Report (MMWR). One proposed solution for prevention of such errors was "careful visual inspection and reading of labels." Confusion of TTCV and PPD is not the only vaccine-related administration error that could be prevented by reading of labels.

METHODS: To give a snapshot of reported errors in vaccine/biologic administration, the sanofi pasteur Vaccine Information Service (VIS) records for a two-year period were reviewed. Calls regarding vaccine administration errors which could have been prevented by "careful visual inspection and reading of labels" were identified.

RESULTS: During a two-year period, almost 500 requests for advice following vaccine administration errors involving more than 20 products were received, of which $33 \%$ were for vaccines administered after the expiry date. Of the remainder, about $13 \%$ gave the wrong dose volume, $25 \%$ used the wrong route, and $8 \%$ either used an incorrect diluent to reconstitute a freeze-dried vaccine or made their own combinations.

CONCLUSIONS: There is potential for adverse consequences when attention is not paid to the information on a vaccine label, such as lack of protection if chemical interaction in an extemporaneously mixed vaccine destroys one or more of the antigens. Many solutions to medication errors have been proposed, including colour-coding of vaccine cartons and labels, provision only of unit dose containers, etc. From the wide spectrum of errors reported, it appears that these would only prevent a portion of the errors. There is no substitute for reading the label, even when not in doubt!

\section{P35}

\section{DEFENDERE SANITAS MILITI: IMMUNIZATION PROGRAM IN THE CANADIAN FORCES}

$M$ Tepper, Department of National Defence; JW Anderson, Department of National Defence

The Canadian Forces Health Services (CFHS) delivers clinical and public health care to the 60,000 members of the Regular Force independent of the provincial/territorial $(\mathrm{P} / \mathrm{T})$ health care schemes. Immunization has long been considered an important pillar of military medicine to protect the Canadian Forces (CF) member and to increase the likelihood of successful completion of the mission. The immunization program covers occupational and nonoccupational disease risks.

While the CF immunization program builds on the civilian norms, there are additional factors that come into play that are seen less often in the civilian sector including:

a. unusual exposure situations, often involving groups of persons (eg, recruit training, deployment) and less commonly used and, at times, unlicensed, vaccines (eg, anthrax vaccine).

b. tension between mission completion and individual protection.

c. conflict between the individual's right to refuse immunization and "protection of the group" and mission completion, which may favour compelling immunization.

d. media attention on the CF's use (or nonuse) of an immunizing agent. e. immunization delivery to members in all $\mathrm{P} / \mathrm{T}$ s and several unusual delivery situations, eg, ships or while on deployment in the developing world. f. quality assurance within about 35 vaccination clinics spread over a very large geographic area.

This presentation will discuss the current immunization approach used by the CFHS and the factors mentioned above.
P36

ALBERTA'S IMMUNIZATION RECOMMENDATIONS FOR
SOLID ORGAN TRANSPLANT RECIPIENTS (SOT) AND
HEMATOPOIETIC STEM CELL TRANSPLANT (HSCT)
RECIPIENTS

R Hamm, Alberta Health and Wellness; E Sartison; S Virani;

C Sarin; E James; J Storek; K Doucette; J Macdonald; W Vaudry;

J Fox; B Lee

INTRODUCTION: Immunization is an important strategy in reducing the risk for vaccine-preventable diseases following solid organ transplantation (SOT) and hematopoietic stem cell transplantation (HSCT). Recommendations for immunization in transplant recipients are complex and the evidence is evolving; however, every effort should be made to appropriately immunize this susceptible group. Currently there are no comprehensive, standardized immunization guidelines for transplant clients in Canada. While some jurisdictions hatasictandards, Alberta undertook a collaborative process to derelongrensive guidelines for the growing transplant population in the Province.

METHODS: In January 2005, a urn.ing group of experts in public health, infectious diseases, transplapt relicine, and medical microbiology convened for preliminary disfdssons. Following a comprehensive review of existing literature and Indetines from Europe and the United States, as well as work from British Columbia and Saskatchewan, immunization schedules were developed by Alberta Health and Wellness with input from the expert wolking group. In January 2006, revised drafts were presented to the Alberta Advisory Committee on Communicable Disease Control (AAC-CDC) for input and approval. The final drafts were circulated to the health regions for an eight-month trial implementation period. Feedback from the regions has been incorporated to address operational issues.

RESULTS: Comprehensive immunization schedules and serology recommendations for transplant recipients are now available for the first time in Alberta. Five separate schedules have been divided to address the separate issues of HSCT and SOT recipients. These categories have been further sub-divided, based on age groups and the option for accelerated schedules. Feedback has been extremely positive.

CONCLUSION: Alberta has successfully developed comprehensive immunization guidelines for transplant recipients. Alberta Health and Wellness will share these guidelines with the Public Health Agency of Canada once they are finalized.

\section{P37}

\section{HEALTH DISPARITIES IN CHICKENPOX, A VACCINE PREVENTABLE DISEASE}

M Russell, University of Calgary; DP Schopflocher, Alberta Health and Wellness; L Svenson

BACKGROUND: Health disparities (differences in health status that occur among population groups defined by specific characteristics) are known to exist in Canada for many diseases and health conditions, including some vaccine preventable diseases. However, few data are available for chickenpox, a vaccine preventable disease for which publicly funded immunization programs have been implemented relatively recently.

PURPOSE: We explore evidence of health disparities due to chickenpox in Alberta, Canada.

METHODS: Chickenpox cases were identified from administrative data from Alberta's universal health care insurance system for 1994-2002. Incident cases (ICD9-CM 052/ IC10-CA B01) were those with the earliest dated utilization of a health service. Crude and age-specific rates were estimated for each year by an indicator of socio-demographic status. Cases were assigned to one of four mutually exclusive and exhaustive premium subsidy groups: No Subsidy, Partial or Full Subsidy, First Nations, and Social Assistance. Individuals who were First Nations were placed in the First Nations group regardless of presence in another group.

RESULTS: Among young children there is a gradient of disparity in chickenpox rates prior to the year in which publicly funded vaccination programs were implemented (highest rates for First Nations, then Social services, health premium subsidy group; lowest for no subsidy group). 
These decline among children over the age of 1 year after 2001, the year in which publicly funded chickenpox vaccination programs were implemented in Alberta. Among children under 1 year of age, there is evidence after 2001 of herd effect for all groups except for First Nations children.

CONCLUSION: Publicly funded vaccination programs may effectively contribute to reduction in disease disparities for vaccine preventable diseases. Further study is required to ascertain why disparities continue for First Nations children.

\section{P38 \\ IS ASPIRATION NECESSARY PRIOR TO AN INTRAMUSCULAR INJECTION?}

E Sartison, Alberta Health \& Wellness (AHW); A Honish, Retired, Past AHW Employee; E James, Past Employee AHW

INTRODUCTION: Aspiration prior to an intramuscular (IM) injection of a vaccine to avoid inadvertent intravascular injection has been an accepted standard of practice for past and current generations of Canadian nurses. Recently, some immunization experts have stated that aspiration prior to an IM injection of a vaccine is not necessary due to a lack of evidence to support the practice while other authorities continue to recommend that vaccine providers perform aspiration.

METHODS: To address this important nursing practice josue, the literature related to IM injection technique and sndion was reviewed and experts were consulted. While there ss volume of literature available, much of the informations is research-based and recommendations were inconsistent. The irou fowhether to discontinue the routine practice of aspiration priby a thy injection of a vaccine because of a lack of evidence to supptrthe practice or whether the lack of evidence indicates that yopir for has been effective in preventing harms remains unresolved

RESULKS: Severat important considerations may be drawn, however, from the hiteryture review and the consultations. These considerations include safety, the importance of careful site selection, attention to injection technique when administering vaccine intramuscularly, incidence of blood vessel entry, route of administration and immunogenicity, syringe technology and liability.

CONCLUSION: The traditional nursing practice of aspiration prior to an IM injection of a vaccine is not evidence-based and questions remain unanswered regarding the practice. These questions present opportunities for nurse researchers to develop evidence in support of maintaining or revising the routine nursing practice of aspiration prior to an IM injection of a vaccine. A request for publication of this paper has been submitted to the Canadian Nurse Association Journal June 2006.

Note: The views in this paper are those of the authors and do not necessarily reflect the views of the Government of Alberta.

\section{P39}

POPULATION-BASED STUDY OF RUBELLA IMMUNITY AMONG PREGNANT WOMEN IN ALBERTA

B Lee, Department of Pediatrics, University of Alberta Hospital (UAH); S Plitt, Public Health Agency of Canada; BE Lee, Department of Pediatrics, UAH; JL Robinson, Department of Pediatrics, UAH INTRODUCTION: There is limited data on rubella immunity in women of childbearing age in Canada. Therefore, it is unclear what proportion of women is at risk of having infants with congenital rubella syndrome. Alberta uses an opt-out strategy in the prenatal screening program requesting physicians not to repeat rubella immunity screen ( $\operatorname{IgG}$ ) for women who have previously tested seropositive. We studied the rate of seroreactivity and inappropriate screening in pregnant women in Alberta. METHODS: Data on all specimens submitted for prenatal screening in Alberta from August 2002 to December 2005 were extracted from the Provincial Laboratory for Public Health. Demographic variables from women who had rubella immunity screening were compared to women who opted-out, and seroreactors were compared to non-seroreactors. The proportion of inappropriate tests (ie, testing of women previously seropositive) was determined. Comparisons between groups were analyzed by Chisquare and t-tests.
RESULTS: Of 159,046 prenatal specimens, $88.3 \%(140,473)$ were screened for rubella immunity. Screened women were significantly younger ( 27.8 years vs. 29.9 years, $\mathrm{p}<0.001)$ and had lower gravidity $(2.3$ vs. 2.8 , $\mathrm{p}<0.001)$ than opted-out women. In total, $8.8 \%$ of specimens tested negative for rubella IgG. Younger women (23.2\% of women younger than 20 years vs. $4.7 \%$ of women $35-39$ years old, $\mathrm{p}<0.001)$ and women from northern Alberta $(11.9 \%$ vs. $8.1 \%, \mathrm{p}<0.001)$ were significantly more likely to be seronegative. Of the 26,045 women who submitted multiple prenatal specimens, $67.8 \%(17,651)$ were screened inappropriately for rubella.

CONCLUSIONS: Younger women and those from northern Alberta are more likely to be seronegative for rubella. The public health significance of women entering their childbearing years with low or undetectable rubella IgG remains to be determined. A large number of women with documented rubella immunity were retested. Automatic cancellation of inappropriate testing in the laboratory might be cost saving.

\section{P40}

\section{ANTICIPATING IMMUNIZATION OF HEALTH CARE WORKERS IN AN INFLUENZA PANDEMIC}

A Kallos, Vaccine Evaluation Center; C Lajeunesse; D Scheifele BACKGROUND: Healthcare workers ( $\mathrm{HCWs}$ ) will be on the front-lines in a pandemic and potentially be significantly affected. Recognizing their importance to sustaining the healthcare system in crisis, Canada's Pandemic Influenza Plan places HCWs first in line to receive vaccine when available. The expectation is that this vaccine will be rapidly deployed, to virtually all eligible HCWs. We examined potential barriers to achieving this objective.

METHODS: We delivered influenza vaccine to HCWs at BC Children's and Women's Hospital in 2005-2006 using a 5-pronged approach to enhance education, accessibility, marketing, service and incentives. Uptake rates were estimated for all workers and confirmed by personal interviews with a large sample of ward staff.

RESULTS: Evaluation was labour intensive for lack of a computerized tally of vaccinees and took weeks to complete. In total, 3082 workers were immunized, about 200 more than in the previous season. Vaccination rates were difficult to measure because 1,993 hospital employees were immunized (of 4,537 on payroll, 44\%) but an additional 1,089 other hospitalbased workers not on payroll (doctors, students, contract workers, etc) were also immunized, of an undefined total. A survey of 992 ward personnel indicated a $71 \%$ vaccination rate, including 114 persons immunized elsewhere and otherwise uncounted.

CONCLUSION: A pressing need exists for a better means of evaluating vaccine uptake in hospital campaigns, the success of which requires improvement. The hospital security database could provide a more comprehensive tally of site workers than do payroll databases. Security badges worn by all staff could be scanned at the immunization visit to populate a real-time database of completed immunizations. Non-vaccinated workers in key roles could be identified for personalized attention, to maximize uptake rates. We plan to test this new approach in coming seasons.

\section{P41}

SOLICITED ADVERSE EVENTS (SAES) FOLLOWING IMMUNIZATION WITH THE 4TH DOSE OF PENTACEL ${ }^{\text {TM }}$ IN BRITISH COLUMBIA, CANADA

D Skowronski, BC Centre for Disease Control; DM Patrick, BC Centre for Disease Control; M Naus, BC Centre for Disease Control; VP Remple, BC Centre for Disease Control; TL Kwindt, BC Centre for Disease Control; P Tsang, sanofi pasteur Inc; F Noriega, sanofi pasteur Inc

BACKGROUND: In Canada, diphtheria, tetanus, acellular pertussis, inactivated poliovirus and haemophilus influenzae type B combination vaccine (DTaP-IPV/PRP-T, Pentacel ${ }^{\mathrm{TM}}$ ) is given at 2, 4, 6 and 18 months. In a retrospective telephone survey with physician validation, solicited adverse events (SAEs) in the 6 months following dose 4 were assessed.

METHODS: Children from an immunization registry in $\mathrm{BC}$ were eligible if they received Dose 4 of Pentacel ${ }^{\text {TM }}$ 6-8 mo earlier, and before the second birthday. SAEs collected via phone interview and verified by primary care 
physicians included any hospital admission (HA), any episode perceived as life-threatening (EPLT), any new condition requiring $\geq 3$ repeat medical visits (RMV), or new diagnosis of specific interest (NDSI) including low blood count (LBC), joint redness/swelling (JRS), asthma (ASTH), diabetes (DIAB) or autism (AUT).

RESULTS: Following household contacts made between September 2003 and April 2004, 3213 families provided complete interview information. Vaccinees (52\% male, $87 \%$ Caucasian) had a median age at immunization of 19 mo; 190 (6\%) had $\geq 1$ identified SAE. 73 had HA (2\%), 41 EPLT (1\%), 118 RMV (4\%) and 34 NDSI (1\%) including 20 ASTH, 9 LBC, 3 AUT ( 2 with signs before Dose 4), 1 JRS, and 1 DIAB. Only 29 reported SAEs were not physician-confirmed. Of the 190 children with an SAE, investigators considered events of $5(0.2 \%)$ to be possibly vaccine-related (red cell aplasia, vomiting, fever, allergic reaction, Kawasaki's disease); $1(0.03 \%)$ probably vaccine-related (febrile seizure); and the remainder to be unrelated.

CONCLUSION: Using a sensitive case definition, we identified a low rate of SAEs with no unusual case clustering around dose 4 of Pentacel ${ }^{\mathrm{TM}}$. Such post-marketing evaluations, with the advantage of large sample sizes and prolonged follow-up, should be encouraged in order to maintain public confidence in ongoing, but especially newly-introduced or revised, vaccine programs.

\section{P42}

\section{INFLUENZA-ATTRIBUTED HOSPITALIZATION RATES AMONG PREGNANT WOMEN, CANADA}

D Schanzer, Public Health Agency of Canada; JM Langley, Department of Pediatrics, Dalhousie University; TWS Tam, Public Health Agency of Canada

BACKGROUND: Although it is recommended that pregnant women with risk factors for complicated influenza receive influenza vaccine, it is not clear if healthy pregnant women are at increased risk for adverse outcomes. We aimed to estimate the rate of hospitalization attributable to influenza for pregnant women and assess the presence of co-morbidities. METHODS: Hospital admissions from 1994-1999 of pregnant women (ICD-9 codes 640-648 and V22, V23) for either a primary respiratory cause or a primary pregnancy-related cause with secondary respiratory diagnoses were extracted from the hospitalization database (Canadian Institute of Health Information). Admissions ending in birth or other termination were excluded. Weekly admissions, stratified by the presence of co-morbid conditions, were modelled as a function of viral activity, seasonality, trend, and holiday effects using Poisson regression with proxies for influenza and other viral activity developed previously for similar agespecific models of influenza-attributed hospital admissions. Person-time of pregnancy was calculated from vital statistics records of births and stillbirths (Statistics Canada).

RESULTS: Approximately 300 hospitalizations of pregnant women per year were attributed to influenza, of which $30 \%$ had chronic co-morbid cardio-pulmonary conditions or diabetes. Asthma was the main co-morbid condition, accounting for $15 \%$ of the influenza-attributable admissions. This burden corresponds to a rate of 155 (95\%CI 140-170) per 100,000 pregnant women. Otherwise healthy pregnant women accounted for over $50 \%$ of these admissions. This burden was relatively constant across multiple influenza seasons of varying severity. Approximately $2 / 3$ of influenzaattributed admissions during pregnancy were coded to pregnancy as the most responsible diagnosis. During the four weeks of peak influenza activity, $60 \%$ of admissions of otherwise healthy pregnant women for acute respiratory conditions could be attributed to influenza.

CONCLUSIONS: Results suggest that influenza is associated with increased rates of admission for healthy pregnant women. As gestational period was unavailable, any expected increase in rates with gestation could not be confirmed.

\section{P43}

\section{SURVEILLANCE OF VARICELLA AND ZOSTER IN CANADA: ARE WE EQUIPPED TO TRACK POST-VACCINE EPIDEMIOLOGY?}

S MacDonald, University of Alberta; W Vaudry, University of Alberta Faculty of Medicine, Department of Pediatrics

BACKGROUND: Routine childhood immunization against varicella zoster virus (VZV) is now funded in most provinces and territories of Canada. While the vaccine is known to reduce varicella (chickenpox) infection, concerns have been expressed that universal immunization programs may lead to detrimental changes in the epidemiology of the disease. Specifically, routine immunization in childhood may shift the burden of varicella disease to the adult population, resulting in higher morbidity and mortality, and may increase incidence of herpes zoster (shingles) infection. To determine whether such changes occur, and whether immunization programs should be amended in response, adequate surveillance of disease and immunization coverage is essential.

PURPOSE: To describe and appraise the current state of VZV surveillance in Canada and to present recommendations for change.

METHODS: Survey of current provincial/territorial and federal VZV surveillance and review of the goals and objectives of effective surveillance systems.

RESULTS: Currently, VZV surveillance in Canada consists of a patchwork of passive and active systems, varying by province/territory. Passive notifiable disease reporting is notorious for underreporting cases, not all provinces/territories report to the national surveillance system, and there is variation in the use of aggregate versus case-based reporting. The Immunization Monitoring Program Active (IMPACT) program, while national in scope, only captures pediatric cases of varicella and zoster which are admitted to selected hospitals across the country. Surveillance of immunization coverage is also deficient in Canada, lacking a reliable and comprehensive method of tracking national rates.

RECOMMENDATIONS: To determine whether changes to immunization policy and programs are needed, action must be taken to improve surveillance. Possible strategies include expanding the IMPACT program to include adult disease, establishing active surveillance in sentinel communities, strengthening and standardizing existing notifiable disease reporting, and moving forward with national immunization coverage surveillance.

\section{P44}

IS THE "AUDIT" AN ACCURATE REFLECTION OF IMPROVED IMMUNIZATION RATES?

J Harder, Public Health; N Chambers, Public Health Nursing; J Ada, MS, Public Health Nursing

BACKGROUND: The introduction of an audit process was implemented in 2004 to meet the obligations of the Ministry of Health and more importantly protect the health of our communities. IWR reports (Impromptu Web Reports) containing data from i-phis (integrated Public Health Information System) were generated monthly for all children in a given cohort living in the Comox Valley. The reports identified children underimmunized at ages 4 months, 6 months, 8 months, 14 months, 20 months, 22 months, and 2 years. The goal was to have children immunized by their second birthday.

OBJECTIVE: To assess whether the implementation of an audit process along with other strategies has increased immunization rates in the Comox Valley.

METHODS: Audit reports focused on the two-year-olds in the Comox Valley. Reports were used to compare rates of immunization for specific agents, from the three years prior to the introduction of the audit process to the present time. The first quarter of 2006 , that is, all children that turned 2 years in January, February, and March 2006 were included.

RESULTS: 1) Audit data of two-year-olds shows improved immunization rates. 2) Reasons for under-immunization were identified through the audit process. 3) New strategies for improving immunization rates were introduced and continue to be evaluated.

CONCLUSION: The audit provides us with an effective tool to assess the number of two-year-olds up-to-date for immunizations according to the 
BCCDC schedule. It provides opportunities to discuss, monitor and evaluate immunization awareness. However, the audit data on its own is not an accurate reflection of improved immunization rates. It is expected that an audit of children at an older age (eg, school entry) would show an increase in the number of children fully immunized in the Comox Valley and would more accurately reflect the strategies implemented to increase awareness and thus immunization rates. Our goals are to increase immunization rates and awareness in our community and in doing so, to ensure parents are fully immunizing their children on time, according to the recommended routine immunization schedule. The audit process, as it evolves, is useful in providing us with opportunities to monitor immunization rates, understand immunization concerns, and to assist us in meeting our goals.

\section{P45}

\section{SELF-REPORTED INTENT TO RECEIVE, AND UPTAKE OF, INFLUENZA VACCINE BY IMMUNIZATION PROVIDERS}

Jane A Buxton, BC Centre for Disease Control; KL Pielak, BC Centre for Disease Control; C McIntyre, BC Centre for Disease Control; VP Remple, BC Centre for Disease Control; DM Skowronski, BC Centre for Disease Control

INTRODUCTION: Health care workers are recommended to receive annual influenza vaccine to protect themselves and reduce influenza transmission to their patients. The objectives of this study among immunization providers (nurses and physicians) in BC were to estimate: 1) the proportion who intend to receive and who report having received influenza vaccine; and 2) factors associated with this intention and self-reported behavior. METHODS: A cross-sectional postal survey based on the Theory of Planned Behaviour was sent to all immunization providers in BC. Associations between future intention to receive and reported receipt of influenza vaccine, and variables achieving a significance of 0.1 on bivariate analyses were modeled using logistic regression.

RESULTS: Completed surveys were received from 335 nurses and 343 physicians (67\% and 22\% response rate respectively). Overall $89 \%$ (95\%CI:86-92\%) of respondents reported intending to receive the influenza vaccine every year; $78 \%$ (95\%CI:74-82\%) reported they receive the vaccine $\geq 75 \%$ of the time. Those with positive direct attitudes (reporting receipt of vaccine for themselves as important, beneficial and good) and positive direct social norms (feeling they should receive the vaccine) compared to those without were 8.3 and 6.2 times respectively more likely to intend to receive influenza vaccine. Those with positive direct attitudes and social norms were 2.5 and 3.2 times more likely to report having previously received the vaccine. Profession (being a nurse or physician) and indirect social norms (opinions of colleagues and employers) were not related to self-reported intention or behaviour regarding influenza immunization.

CONCLUSIONS: Almost $90 \%$ of immunization providers intend to receive annual influenza vaccine. Although self-reported uptake was closer to $80 \%$, this rate is higher than that measured in health care workers in acute and long-term facilities. Positive attitudes among health care workers towards receiving the vaccine themselves may be important in their recommending it to others. Health promotion initiatives for health care workers should target individual perceptions, as opinions of others may have less effect on their own intention or behaviour.

\section{P46}

CERVICAL CANCER SCREENING AND EVENTUAL HPV VACCINE ACCEPTABILITY: AN EXPLORATORY SURVEY IN CANADIAN ADULTS

V Gilca, Laval University Hospital Center; B Duval, Quebec Institute of Public Health; V Gilca, Laval University Hospital Center; F Lavoie, Laval University Hospital Center; M Ouakki, Laval University Hospital Center

BACKGROUND: The Pap test has been used for cervical cancer screening for more than four decades. A human papillomavirus (HPV) vaccine has been approved for use in Canada and will soon commercially available. These two preventive interventions should now be considered simultaneously. Support from both men and women will be important for the successful combination of these interventions.
OBJECTIVES: 1) To assess practices, beliefs, and attitudes toward Pap test screening and eventual HPV immunization; 2) To identify sociodemographic factors for Pap screening and vaccine acceptability.

METHODS: In 2006, 500 adults were invited to participate in a telephone survey in the region of Quebec City (urban and rural population, 600 000), Canada. Pap test and HPV information was provided before soliciting opinions.

RESULTS: 471 adults (18-69 yo) answered the questionnaire, the mean age was 45 years, $67 \%$ were female, and $65 \%$ had postsecondary education. Eighty-six percent of women had undergone at least one Pap test in their life, $64 \%$ in the last year, and $17 \%$ from 1 to 3 years ago. Among screened women, the test had been performed in the last three years in $100 \%$ of $18-30$ year-olds, but only in $67 \%$ of $60-69$ year-olds $(\mathrm{P}<0.0001)$. Only $15 \%$ of respondents had heard of HPV. Eighty-five percent agreed that HPV vaccines could prevent cervical cancer, $71 \%$ that the vaccine has to be administered before sexual debut, $84 \%$ would recommend vaccination to their daughters and nieces. Among respondents $<25$ years, $91 \%$ would agree to receive the vaccine if it is free, but only $62 \%$ would agree to pay $\$ 100 /$ dose.

CONCLUSIONS: Older women are less screened for cervical cancer than young women. A small proportion of the adult population have heard about HPV. The majority of respondents would recommend or are ready to receive the HPV vaccine, but the vaccine cost could prevent its acceptability and introduce preventive care inequity.

\section{P47}

KEEPING THEM IN: PARTICIPANT RETENTION IN LONG TERM IMMUNOGENICITY - BOOSTER STUDIES

V Gilca, Laval University Hospital Center; C Couture, Public Health Research Unit, Laval University Hospital Center; F Lavoie, Public Health Research Unit, Laval University Hospital Center; S Auger, Public Health Research Unit, Laval University Hospital Center; V Gilca, Public Health Research Unit, Laval University Hospital Center

BACKGROUND: The duration of protection provided by a vaccine is a major unknown when a new immunization program is implemented. Measuring the protection of vaccinated cohorts at a future date is an effective evaluation strategy. It is of the utmost importance to retain a high proportion of participants to minimize bias and achieve the study objectives. METHODS: We designed two 15-year cohort studies to document the protection induced by Engerix- $\mathrm{B}^{\circledR}$ and Recombivax-HB ${ }^{\circledR}$ and the effect of a booster given 5,10 or 15 years later. Specific strategies were used to minimize the loss of parental support and child participation. This included regular communications (letters, birthday cards, newsletter), the use of specific themes (the importance of the research project objectives, the contribution to the progress of science) and the provision of services (knowledge of immune status). A loss of $5 \%$ of subjects per year was anticipated.

RESULTS: Two cohorts of 1200 and 1126 8-10 year-old children were enrolled in 1995-1996 in Recombivax and Engerix studies respectively. After 10 years, 940 (83.5\%) and 987 (82.3\%) subjects remain in the study. The average loss of participants per year was less than $2 \%$. In the Recombivax study, the highest number of withdrawals - 149 (13\%) were at year 5-6, when 3 blood samples were taken and one booster dose administered. In the Engerix study, two critical periods were observed: year zero, $71(6 \%)$ and year 5-6, $111(9 \%)$ withdrawals. Slight dissimilarity in study design may explain these differences.

CONCLUSIONS: Maintaining high rates of participation in long term studies is a big challenge. Specific, age-dependent interventions improves study participation. 


\section{P48}

ADVERSE EVENTS FOLLOWING ROUTINE INFANT/TODDLER IMMUNIZATIONS, BRITISH COLUMBIA, 2004/05

S David, BC Centre for Disease Control; M Naus, BC Centre for Disease Control; Vaccine Safety WG, British Columbia Immunization Sub-Committee

INTRODUCTION: As effective immunization reduces the effects of vaccine-preventable diseases, concern is shifting toward risks associated with vaccines. There is a paucity of information on adverse events following immunization (AEFI) that can be expected following the current infant/toddler schedule in which $2-4$ vaccines are administered concurrently. The purpose of this analysis was to estimate the proportion of children immunized with the recommended infant/toddler vaccines in $\mathrm{BC}$ who reported AEFI through the passive surveillance system and describe their events and outcomes.

METHODS: AEFI reports following 2-, 4-, 6-, 12- and 18-month immunizations administered between July 1, 2004 and June 30, 2005 were extracted from BC's Integrated Public Health Information System (iPHIS) for 14/16 Health Service Delivery Areas. The percent of children immunized at each milestone who reported AEFI, and the percent who reported specific signs, symptoms and outcomes, were calculated using denominators from iPHIS.

RESULTS: AEFI were reported for $0.26 \%$ (95\% CI=0.19-0.32\%), $0.28 \%$ (95\% CI=0.21-0.35\%), $0.17 \%$ (95\% CI=0.12-0.22\%), $0.49 \%$ $(95 \% \mathrm{CI}=0.41-0.57 \%)$ and $0.21 \%(95 \% \mathrm{CI}=0.16-0.26 \%)$ of infants/ toddlers immunized with the 2-, 4-, 6-, 12- and 18-month vaccines, respectively. The most frequently-reported signs and symptoms were screaming/ crying following the 2 -month doses $(0.09 \%$ of immunized), fever following the 6 -month doses $(0.17 \%$ of immunized) and rash/hives following the 4-month, 12-month and 18 -month doses $(0.11 \%, 0.36 \%$ and $0.08 \%$ of immunized, respectively). Of the 131,740 infants/toddlers immunized, one case of anaphylaxis (7.6 cases per million immunized), one case of encephalopathy and no deaths were reported.

CONCLUSIONS: The type and rate of AEFI reported varied with the dose number and may reflect age- and dose-specific incidence of causally associated events, age-related differences in coincidental behaviours, events unrelated to immunization and events that cause concern in parents. Very few serious events were reported. Establishing expected rates of AEFI following the routine series can assist in further enhancing AEFI surveillance, including automated aberration detection.

\section{P49}

INVESTIGATING USER PERCEPTION, CHANGE MANAGEMENT AND BARRIERS IN IMPLEMENTING A NEW PROVINCIAL INVENTORY MANAGEMENT SYSTEM

C LeBlanc, Nova Scotia Department of Health

INTRODUCTION: The purpose of this abstract is to examine the relationship between implementing an automated inventory management system within the province of Nova Scotia and user response to the change. STUDY: Inventory management of biologicals is an integral part of any immunization program, which is why the decision to move from a paper inventory system to an automated system (SAP) was decided upon in late 2004. This study examines various milestones, ranging from the implementation of the system, a phase II rollout, the current state of the system, as well as the future of the system. It analyzes the change management, user perception, and barriers that were experienced during this time. The data collected for this review includes the lessons learned of implementation, an end user questionnaire regarding system usage, training and phase II issues, as well as planning documents regarding future state.

CONCLUSIONS: After preliminary examination all of the data key conclusions will include:

- Initial resistance to change lead to user acceptance that ranged from short- to long-term

- Inventory management is a business process with clear financial components/objectives

- The support of the business process and availability of management information has greatly improved and set the stage for further improvement of the governance of vaccine inventory use and management between the Department of Health and the various District Health Authorities.

- Detailed project planning including change management alleviated the foreseen user acceptance issues.

- Experience with the current tool has paved the road for better requirements discussions for a new system and has decreased anxiety levels for the users as well as created a setting for a uniform provincial approach.

- Experiences learned from the project being useful in helping plan the implementation of any Immunization registry and of other vaccine-related databases that will be introduced as part of the Pan Canadian Infoway solution.

\section{P50 \\ TEMPORAL TRENDS IN ADVERSE EVENTS REPORTED FOLLOWING IMMUNIZATION (AEFI) BY AGE OVER A SIX-YEAR PERIOD IN CANADA}

N Ahmadipour, Public Health Agency of Canada; BL Law, MD

INTRODUCTION: Canadian post-marketing surveillance of vaccines through passive and active programs collects reports of AEFIs by health care providers, manufacturers and consumers, as well as a network of 12 pediatric hospitals across the country that actively monitors for selected serious AEFIs.

OBJECTIVE: To show temporal trends in AEFIs by age over a six-year period from 2000 to 2005.

METHODS: All AEFI reports with immunization between 01/01/00 and $31 / 12 / 05$ were retrieved. Cases were then categorized into three age groups: infants $(<1$ year); children $(1-<17)$; and adult $(\geq 17)$. Most common and serious AEFIs were selected and compared across the years and ages.

RESULTS: Of 26,540 total reports, 12\% involved infants, $41 \%$ children, $47 \%$ adults. There were 5,431 reports in 2000 that gradually decreased to 3,223 in 2005. Overall, five most frequently reported AEFIs in order of frequency included fever (24\% of all reports), severe local reaction $(17 \%)$, rashes $(14 \%)$, oculorespiratory syndrome (ORS, 13\%), and allergic reaction (12\%). However, analysis by age and year showed: ORS was common in adults and only in 2000 through 2002; and severe vomiting/diarrhea were among the top 5 common AEFIs in children and infants, in contrast to allergic reaction in adults. Of serious AEFIs, GBS and Bell's palsy were mainly reported in adults across the years; anaphylaxis mostly in adults and then children; convulsions, meningitis/encephalitis/encephalopathy, and thrombocytopenia mainly in infants and children (higher numbers in children, but same proportion of the two age groups). HHE and persistent crying are limited to infants.

DISCUSSION: Temporal trends in AEFIs can be used to describe and compare distribution of events across regions and detect any unusual or unusually increased in frequency for further investigation/action. These data can be used to develop programs to facilitate detection of signals related to newly introduced vaccines which primarily target specific age groups.

\section{P51}

\section{USE OF VARICELLA VACCINE DURING A CHICKENPOX OUTBREAK IN A SCHOOL SETTING WITH INTERNATIONAL STUDENTS}

C Springinotic, Vancouver Island Health Authority; M Gilbert, Vancouver Island Health Authority; A Shaw, Vancouver Island Health Authority; M Naus, BC Centre for Disease Control

BACKGROUND: In BC, there has been a recent expansion of eligibility for varicella vaccine for children, adolescents and adults at opportune health encounters, including use during post-exposure encounters. On April 4th 2006, the department of Epidemiology and Disease Control, Vancouver Island Health Authority, received a report of four cases of clinically diagnosed varicella disease among student residents (aged 17-19 years) at a school setting with 207 international students. This poster will describe an outbreak of chickenpox in an international school setting. The use of varicella vaccine and results of serologic testing during a vaccination 
campaign conducted during this outbreak. On April 6 2006, varicella vaccine was offered to susceptible students who had no documented history of varicella vaccine or history of disease. Susceptibility at the time of presentation for vaccination was confirmed by testing for varicella immunity (VZV IgG). The goal of the vaccination campaign was to: a) prevent additional cases among susceptible students; b) prevent exportation of disease to the local community and/or a student's country of residence.

RESULTS: 8/207 (3.8\%) students presented with varicella disease between March 4th and April 28th 2006. One case was laboratory confirmed (VZV IgM Reactive). Index case was a Canadian female with previous history of mild disease but was non-immune based on serology collected prior to college admission. Country of residence of cases: Canada (2), Central America (2), South America (1), India (1), Slovak Republic (1) and Kenya (1).

DISCUSSION: Varicella outbreaks may occur in adolescent populations in closed settings. Use of vaccine may have assisted in controlling the outbreak. 10/12 (83\%) of students susceptible to varicella did not develop disease following the immunization clinic. Client report of susceptibility may or may not be an accurate indicator of immunity and serology is necessary to determine who is or is not susceptible.

RECOMMENDATIONS: In this setting, immunization records and student disease history should be reviewed annually. Serologic testing followed by vaccination should be offered to students identified as potentially susceptible.

\section{P52 \\ MODELLING HUMAN PAPILLOMAVIRUS VACCINE EFFECTIVENESS: QUANTIFYING THE IMPACT OF PARAMETER UNCERTAINTY}

N Van de Velde, Laval University; M Brisson, Unité de recherche en santé des populations, Université Laval, Quebec; MC Boily, Department of Infectious Disease Epidemiology Imperial College United Kingdom

INTRODUCTION: Mathematical models are based on assumptions, which inevitably embed a certain level of uncertainty. Quantifying the parameter uncertainty is particularly important when molelling Human Papillomavirus (HPV) vaccine effectiveness, as the naturgt history of infection and disease is complex, and age and type-specific data remain scarce and incomplete. The aim of this study was to predict the ippact of HPV-6/11/16/18 vaccination in Canada, accounting for parane uncertainty.

METHODS: An extensive fitting proce was conducted on a cohort model using multivariate Latin Hyperas Sampling, which identified 164 posterior parameter combinztions of 200,000 prior parameter combinations) that fit simuly anf 1 HD type-specific incidence and prevalence data for infectop and Squamous Cell Carcinoma (SCC). RESULTS: Based on these posterior parameter sets, the model predicts that vaccinating 12-year-old girls (vaccine efficacy $=95 \%$, no waning) would reduce theiy lifetime risk of HPV infection, CIN1, CIN2/3 and SCC by $21 \%$ ( $80 \%$ credibility interval: 17,29$), 24 \%$ ( $80 \%$ credibility interval: $17,31), 49 \%$ (80\% credibility interval: 36,60$)$ and $61 \%$ (80\% credibility interval: 47,73$)$, respectively. If vaccine efficacy is reduced or vaccine protection is assumed to wane, uncertainty surrounding predictions widen considerably.

CONCLUSIONS: Model results suggest that the overall Canadian incidence of HPV infection, CIN and SCC are likely to be significantly reduced by vaccination against HPV. Important priorities for future research are to understand the role of natural immunity and to measure the duration of vaccine protection as results were most sensitive to these parameters. Using multiple parameter combinations fitting the different disease stages allows to quantify the parameter uncertainty surrounding model predictions and should be performed when evaluating the potential cost effectiveness of HPV vaccination programs.

\section{P53}

\section{PROFILE OF NURSES' IMMUNIZATION PRACTICES IN} COMMUNITY HEALTH CARE SETTINGS

G Petit, Université de Montréal; L Gauvin, Université de Montréal, Québec; M Letellier, Direction de santé publique de Montréal, Québec; N Boulianne, Institut national de santé publique du Québec, Québec; L Valiquette, Direction de santé publique de Montréal, Québec; C Larue, Université de Montréal, Québec; C Guimond, Direction de santé publique de Montréal, Québec INTRODUCTION: Recent studies have highlighted that selected subgroups of health professionals harbour doubts about immunization. In this regard, nurses report more doubts in comparison to physicians (Dionne et al, 1999; 2001; Petrovic, Roberts and Ramsay, 2001; St-Amour et al, 2004). Vaccine coverage rates can only be achieved if all health professionals adopt practices that effectively promote vaccination. The purpose of this presentation is to describe the immunization-related professional practices of nurses working in community health care settings who hold immunization responsibilities towards children aged 0 to 5 years.

METHODS: Based on expert consultations, a conceptual description of the repertoire of immunization practices of nurses was established. A questionnaire was developed and pre-tested among a sample of immunizing nurses $(n=8)$ and then was mailed to all Quebec nurses working in community health care settings and who had performed immunization tasks among 0 to 5 year old children in the previous 6 months.

RESULTS: A total of 994 (out of 1252) eligible nurses returned a valid questionnaire. Expert consultations showed that nurses' immunization practices can be regrouped under 35 distinct practices. Examination of nurses' data showed that some immunization practices were systematically adopted by almost all nurses whereas adoption of others varied substantially across individuals. For example, $95 \%$ of nurses reported always "offering to give 2 recommended injections to a child during the same visit", but "offering to give 3 or 4 recommended injections to a child during the same visit" was reported to be always done respectively by $19 \%$ and $6 \%$ of sample. CONCLUSION: We conclude that there is a gap in what experts believe are ideal immunization practices and what nurses report being able to perform in community health care settings. Further analyses will provide more information of the variation of practices across settings and individuals.

\section{P54 \\ PREVALENCE OF HUMAN PAPILLOMAVIRUS INFECTION IN CANADA: A SYSTEMATIC REVIEW}

B Pham, Department of Health Policy, Management \& Evaluation; M Do, Statistics \& Epidemiology, GlaxoSmithKline Canada; A Anonychuk, Statistics \& Epidemiology, GlaxoSmithKline Canada INTRODUCTION: Worldwide HPV distribution varies greatly across populations. Such heterogeneity has not been systematically examined in Canadian studies. We conducted a systematic review to consolidate Canadian data on HPV prevalence and associated risk factors.

METHODS: MEDLINE (01/1966-12/2005) and EMBASE (01/1980 to 12/2005) were searched ("human papillomavirus" AND "Canada") for studies reporting HPV prevalence data. Article screening, reviewing, and data abstraction (ie, study, participant and test characteristics, prevalence and risk factors) were conducted independently by two reviewers. Risk factor data were meta-analyzed using random-effects (RE) model, as appropriate. RESULTS: Seventeen studies (published 1997-2005) were included from 206 citations. The studies ranged from 44 to 2098 participants (median 498). Population-based HPV prevalence estimates were obtained from studies conducted in Ontario $(n=1,111)$, Winnipeg $(n=1,477)$, Newfoundland $(n=2,098)$, and Nunavut $(n=1,855)$. HPV infection was diagnosed with PCR ( $\mathrm{n}=11$ studies), Hybrid-Capture II ( $\mathrm{n}=4$ studies) and both $(n=2)$. Oncogenic HPV prevalence was estimated from the Ontario study: $16 \%$ in ages $15-19$, peaked at $24 \%$ in age $20-24$, and $16 \%, 12 \%$, $10 \%,<10 \%$ and $12 \%$ in ages $25-29,30-34,35-39,40-59$ and $>60$. Across regions, HPV prevalence among young women varied from $17 \%$ in Newfoundland (HC-II, ages 18-24), 33\% in inner-city Winnipeg (PCR, $73 \%$ aged $<30$ ), and $42 \%$ in Nunavut (HC-II, ages $13-20$ ). HPV prevalence was $22 \%$ among university students in Montreal (oncogenic PCR, 
$\mathrm{n}=1$ study/621 participants), 33\% in an HIV cohort (PCR, $n=2 / 598)$, and $53 \%$ in women at high risk for STI (PCR, $n=1 / 274)$. Preliminary data on reported risk factors for oncogenic HPV infection included single versus married ( $\mathrm{n}=2$ studies/2588; RE adjusted odds-ratio 1.35 [95\% CI: 0.94, $1.78]$ ), and $>2$ sexual partners in the previous year $(n=2 / 2588$; AOR 1.21 [0.82, 1.62]), among others.

CONCLUSION: The burden of oncogenic HPV infection varied across populations in Canada. This consolidated data would facilitate the planning of immunization programs.

\section{P55}

\section{A REVIEW OF THE MANITOBA HEALTH INFLUENZA IMMUNIZATION}

\section{Mclvor}

INTRODUCTION: This University of Manitoba Master's of Nursing practicum reviewed the Manitoba Health 2004 Influenza Immunization Program Planning Model against existing in-house planning documents and year end data and an evidence-based model was proposed for 2005. In Manitoba, remuneration for immunization provision by both private physicians and the Public Health Authorities is based upon data entry into the Manitoba Immunization Monitoring System (MIMS) database.

METHODS: The student/investigator functioned as an immunization specialist in the Communicable Disease Control Unit of Manitoba Health, using a participant-observer research strategy. The predicted components of the model were reviewed including: population to be immunized in the various target groups, coverage expected to be achieved, vaccine wastage, vaccine costs, and administration of vaccine costs. Supporting literature was found for the framework of the model, the key variables to be considered, coverage rates, and vaccine wastage.

RESULTS: There were 318,500 doses distributed to Manitoba immunizers in the 2004/05 influenza immunization season and $72 \%$ were entered as administered in the provincial immunization registry. Published guidelines from both the World Health Organization (WHO) and the General Alliance for Vaccines and Immunizations (GAVI) predict this large volume of "unaccounted for" vaccine. The new infant immunization program achieved at least $40 \%$ coverage.

CONCLUSIONS: The recommended 2005 model included more accurate target group population estimates, planning for a reserve stock and vaccine wastage, and increased coverage rates in infants. It is expected that this improved model will have applicability with other vaccines and in other jurisdictions.

\section{P56}

REVIEW OF CAUSALITY ASSESSMENTS OF ADVERSE EVENTS FOLLOWING IMMUNIZATION BY THE CANADIAN ADVISORY COMMITTEE ON CAUSALITY ASSOCIATION: 1994 TO 2006

B Law, Public Health Agency of Canada (PHAC); N Ahmadipour, Immunization and Respiratory Infections Division, PHAC, Ottawa INTRODUCTION: The Advisory Committee on Causality Association (ACCA), an independent volunteer panel with expertise in clinical medicine, infectious disease, neurology, immunology and epidemiology, was initiated in 1994 by Health Canada's Childhood Immunization Division to analyze, classify and interpret selected serious or unusual adverse events following immunization (AEFI) and to recommend further investigations as needed. Case selection criteria include fatalities, anaphylaxis requiring medical therapy or resuscitation, neurological AEFI, thrombocytopenia and other severe or unusual events requiring hospitalization. The purpose of this report is to profile all cases reviewed by ACCA.

METHODS: All reports entered into the Canadian Adverse Event Following Immunization Surveillance System database from January 1994 through July 2006, for which a causality term was present, were selected for review.

RESULTS: Among a total of 58,273 AEFI reports received from 1994 through July 2006, 3420 (5.9\%) met the selection criteria for ACCA review. Of these 1150 (33.6\%) were flagged for review and 606 (17.7\%) were fully reviewed by ACCA. Reasons for not assessing 544 cases included insufficient detail for $260(47.8 \%)$, decision to review changed for $250(46.0 \%)$ and review still pending for 34 (6.2\%). Among 606 fully reviewed cases the distribution was as follows for: gender: $44.3 \%$ female; age: $56 \%<2$ years, $9 \%$ 2 to $<7$ years, $11 \%$ school age, $18 \% 18$ to $<65$ years and $6 \% 65$ years and older; vaccine: $26 \%$ pertussis containing, $21 \%$ bacterial polysaccharide, $20 \%$ measles containing, $12 \%$ influenza, $4 \%$ oral polio, 3\% BCG, $14 \%$ other; medical attention: $69 \%$ hospital, $7 \%$ emergency room, 11\% outpatient clinic, $13 \%$ none or not indicated; outcome: $5 \%$ fatal, $14 \%$ residual effects, $8 \%$ recovering, $62 \%$ recovered, and in $11 \%$ not reported; AEFI type: neurologic $48 \%$, anaphylaxis $4 \%$, thrombocytopenia $11 \%$, other $37 \%$; causality rating: very likely/certain: $13 \%$, probable $14 \%$, possible $20 \%$, unlikely $20 \%$, unrelated $28 \%$ and unclassifiable $5 \%$.

CONCLUSION: Causality assessment is an exacting process requiring time, expertise and clinical case details. The work of ACCA has directly contributed to vaccine safety in Canada over the last 13 years.

\section{P57}

PUBLISHED DATA UNDERESTIMATES THE BURDEN OF HEPATITIS A INTERVENTION TRIGGERED BY INFECTED FOOD-HANDLERS

B Pham, Department of Health Policy, Management \& Evaluation; B Pham, Health Policy Management \& Evaluation, University of Toronto; B Duval, Centre de Recherche du CHUQ, Universite Laval; G De Serres, Centre de Recherche du CHUQ, Universite Laval; V Gilca, Centre de Recherche du CHUQ, Universite Laval; L Vrbova, Ontario Ministry of Health \& Long Term Care, Toronto, Ontario; A Anonychuk, University Health Network, Toronto, Ontario; M Krahn, Health Policy Management \& Evaluation, University of Toronto; D Moher, Chalmers Research Group, Children's Hospital of Eastern Ontario

INTRODUCTION: In countries with low hepatitis A endemicity, infected food handlers are the source of most reported foodborne outbreaks. In Canada, accessible data repositories of infected food handler incidents are not available. We undertook a systematic review of such incidents to evaluate the extent of viral transmission through food contamination and the scope of post-exposure prophylaxis (PEP) interventions. METHODS: A systematic search of MEDLINE and EMBASE was conducted to identify published reports of incidents in Canada. An expanded search of a news repository (ie, transcripts from newspapers and newscast) was also conducted to identify the location and timing of an incident and to retrieve the related report by contacting local public health. Data pertaining to case identification, public health risk, PEP interventions, and associated costs was independently abstracted by two reviewers and summarized according to incidents with and without large PEP interventions. RESULTS: A total of 16 incidents were identified between 1998-2004. Incidents requiring public notification were not infrequent, approximately 3 per year. Only $12.5 \%$ of incidents were described in published reports, indicating that published data significantly underestimated the number of incidents and PEP interventions. Data pertaining to the remaining incidents was unpublished, sparse and highly dispersed at the local public health level. Six of the 16 incidents required large PEP interventions to immunize on average 5000 potentially exposed individuals. Secondary transmission was low. Characteristics of incidents requiring large PEP interventions included potentially infectious food handlers working with uncooked food for a prolonged duration in high-volume grocery stores located in high-density urban areas.

CONCLUSION: Infected food handlers with HAV requiring public notification are not infrequent in Canada. Published data severely underestimated the burden of PEP intervention. Better and consistent reporting at the local and national level and a national data repository should be considered for the management of future incidents. 


\section{P58}

COST ANALYSIS OF PUBLIC HEALTH DELIVERY OF CHILDHOOD IMMUNIZATIONS IN NOVA SCOTIA

S Sarwal, Nova Scotia Health Promotion and Protection; L Kumaranayake, Dalhousie University; J Scott, Nova Scotia Department of Health Promotion and Protection; S Sarwal, Nova Scotia Department of Health Promotion and Protection; A Coombs, Nova Scotia Department of Health Promotion and Protection; E Holmes, Nova Scotia Department of Health Promotion and Protection; I Sketris, Dalhousie University

INTRODUCTION: Childhood immunizations are one of the most costeffective health interventions. However, little is known about the variation in costs in delivering vaccines across service providers and across geographic regions. In Nova Scotia, childhood immunizations are delivered by both public health services and by family physicians. The purpose of this project is to develop a costing framework that will be used to estimate costs of public health delivery of childhood immunizations in Nova Scotia. METHODS: The cost analysis framework will be piloted in the Colchester East-Hants Health Authority in Nova Scotia. The analysis will be performed from the perspective of the health care provider. An incremental cost approach will be used to assess the cost of undertaking childhood immunization activities that are performed in addition to existing services, such as well-child care. The total provider costs will be estimated using a combination of an ingredients-based costing methodology, whereby quantities of resources are multiplied by their respective prices, and a step-down methodology, where total costs are allocated to activities according to the amount of time spent on each activity. Sensitivity analyses will be used to examine the influence of data uncertainty on cost results.

RESULTS: Recurrent and capital costs will be gathered to calculate total cost. Average cost will be calculated using output measures compiled from public health offices. Average costs of immunization will be reported for each vaccine on a per dose basis. Average and incremental costs will be used to assess the relative efficiency of delivering multiple vaccines per visit. CONCLUSIONS: The results of this study will provide evidence that will help policy-makers evaluate costs of implementing new programs for publicly funded vaccines. These findings may be used as the basis of a comparison of the cost of public health and physician delivery of childhood immunizations in Nova Scotia.

\section{P59}

\section{DECISION-AID TO INCREASE INFLUENZA VACCINATION AMONG FRONT-LINE HEALTHCARE WORKERS}

A McCarthy, Division of Infectious Diseases, The Ottawa Hospital; SM Sullivan, Élisabeth Bruyère Research Institute; J Sutherland, The Ottawa Hospital; A O'Connor, Ottawa Health Research Institute; LW Chambers, Élisabeth Bruyère Research Institute; J Villeneuve, SCO Health Service; FD Knoefel, SCO Health Service; D Baker, SCO Health Service; D Gallant, St Francis Xavier University; P Arnold, Ottawa Public Health

INTRODUCTION: Influenza vaccination is recommended for healthcare workers to prevent transmission of influenza and to keep essential services workers healthy. Despite the effectiveness of the influenza vaccine, uptake among front-line healthcare workers falls well-below target levels of $90 \%$. An evidence-based tool that can be used by front-line healthcare workers to make informed, values-based decisions regarding influenza vaccination will be presented.

METHODS: Decision-aids are interventions designed to help people make deliberate choices by providing personalized information about relevant treatment options (eg, vaccination, anti-virals)and decision outcomes (eg, side effects, influenza infection, transmitting influenza to patients). A multidisciplinary team involving occupational health, public health, researchers and health professionals produced the new decision aid, adhering to scientific standards outlined by the Collaboration on International Patient Decision-Aid Standards (IPDAS). To ensure high-quality decision-aids, the standards address twelve domains related to development, content and evaluation (eg, systematic development process, evidence-based, balanced, values clarification, plain language, decision quality).
RESULTS: The decision-aid provides complete information about all available options for influenza prevention, including vaccination, antivirals and refusing both vaccine and anti-virals. Development of the decision-aid involved focus groups and surveys of front-line workers and managers. Probabilities on side effects and how well each option prevents influenza were obtained from a systematic literature review and are provided in a user-friendly format. The decision-aid allows front-line workers to express their personal values by indicating which advantages and disadvantages of each option are most important to them. The decision-aid was reviewed by Canadian infectious disease experts and front-line healthcare workers and is ready for pilot testing.

CONCLUSIONS: Decision-aids may be helpful for front-line workers when vaccination is mandatory and for other public education purposes. The new influenza decision-aid provides a comprehensive and user-friendly approach to obtaining consent. As well, it assists front-line healthcare workers to make an informed values-based decision about influenza vaccination.

\section{P60}

IMMUNIZATION PROVIDER KNOWLEDGE: A SURVEY OF BRITISH COLUMBIA NURSES AND PHYSICIANS

C McIntyre, British Columbia Centre for Disease Control (BCCDC); JA Buxton, BCCDC; VP Remple, BCCDC; B Halperin, Dalhousie University; K Pielak, BCCDC; DM Skowronski, BCCDC

OBJECTIVES: To determine the level and correlates of Immunization Providers' (IPs) overall knowledge related to provision of immunizations, and specific knowledge related to vaccine preventable diseases (VPD), vaccines, and vaccine administration and handling (VAH) practices.

METHODS: Participation in a cross-sectional postal survey was offered to all identified IPs in BC. The survey elicited data on demographics, practice characteristics and knowledge related to VPD, vaccines, and VAH. High knowledge scores were defined as those achieving $80 \%$ or greater correct answers within each topic. Logistic regression modeled the association between high knowledge scores and variables of interest achieving a statistical significance of 0.1 or less on univariate analyses.

RESULTS: 253 RNs and 285 MDs (50\% and 23\% response rate, respectively) completed the survey. Respondents had spent an average 15.4 years administering vaccines, and $55 \%$ provided at least 10 paediatric vaccines per month. $27 \%$ (59\% MDs and 12\% RNs) were university-affiliated, and $72 \%$ (95\% RN and 52\% MDs) had received post-academic immunization training. The average score was $71 \%$ on the overall questionnaire and $80 \%$ for VPD, $70 \%$ for vaccines and $67 \%$ for VAH. High overall score was associated with nursing profession (OR 20.6, 95\%CI 10.9, 38.7). High vaccine scores and VAH scores were also associated with nursing profession (OR 9.7, 95\%CI 6.3, 14.9; and OR 41.7, 95\%CI 10.1, 173.1 respectively). No variable was significantly associated with high VPD scores

CONCLUSIONS: Education specific to vaccines and VAH will support immunization providers and enhance immunization services. Profession is the factor most associated with high knowledge scores in this survey. This may be a reflection of practice in British Columbia, where nurses receive immunization training before delivery of immunization services, and where $60 \%$ of all immunizations are provided by public health nurses.

\section{P61}

ACCEPTABILITY OF HUMAN PAPILLOMAVIRUS VACCINATION AMONG ADOLESCENT GIRLS AND THE PARENTS OF ADOLESCENT GIRLS

M MacFarlane Conrad, Canadian Centre for Vaccinology; S McNeil, Canadian Centre for Vaccinology, Halifax, Nova Scotia; B Halperin, Canadian Centre for Vaccinology, Halifax, Nova Scotia

BACKGROUND: Human papillomavirus (HPV) is the cause of genital warts and of over $95 \%$ of cervical cancers. Recently, development of highly effective HPV vaccines has created potential for primary prevention of cervical cancer. To achieve maximal reduction in HPV infection and disease, vaccines must be given prior to sexual debut and will therefore be targeted at pre-adolescent girls and require parental consent; successful implementation of HPV vaccination programs will demand parental buy-in. 
Understanding the knowledge, attitudes, and beliefs of Canadian adolescents and parents regarding HPV infection and its prevention will be critical to inform decision-making around HPV vaccination and screening programs, and to identifying the educational needs of the Canadian public. METHODS: A 63-item self-administered survey of knowledge, attitudes, and beliefs regarding HPV vaccination was developed and validated for test-retest, inter-rater and content validity. The survey was distributed to parents of girls aged 9-17, and teen girls aged 13-17 in Halifax, Nova Scotia. Data were analysed using Fisher's exact test for significance, and predictive models were run using PROC GENMOD in SAS ${ }^{\circledR}$ v.8.2.

RESULTS: To date, 147 surveys have been collected: 83 parent surveys and 64 adolescent surveys. Of teen respondents, $29.6 \%$ were sexually active: The mean age of sexual debut was $14.5 \mathrm{yrs}$ (range $12-17 \mathrm{yrs}$ ). Only $52 \%$ of parents had heard of HPV while $58 \%$ of teens knew of HPV. Perception of HPV risk was nearly identical between parents and teens. $71 \%$ of teens would receive an HPV vaccine, while $77 \%$ of parents would allow their daughters to receive the vaccine. Parents who expressed uncertainty wanted more safety and efficacy information. Among teens, the recommendation of a doctor was most predictive of vaccine acceptance, while in parents, the safety of the vaccine was the most important predictive factor. Most parents reported willingness to pay for the vaccine.

CONCLUSIONS: HPV vaccines are likely to receive broad acceptance by Nova Scotia teens and their parents. In order to ensure universal protection, the vaccine should be given prior to age 12 years. Maximizing acceptance will require public endorsement of the vaccine by health care providers, recommendations of personal physicians to their young patients, and public education campaigns providing vaccine safety and efficacy data to parents.

\section{P62}

MODELLING HAV AND HBV IMMUNIZATION SCENARIOS AMONG NEW ADMISSIONS TO FEDERAL PENITENTIARIES IN CANADA: A COST-BENEFIT ANALYSIS

J Smith, Correctional Service Canada; C Sevigny, Public Health

Agency of Canada; E Ellis, Public Health Agency of Canada;

F Bouchard, Correctional Service Canada

BACKGROUND: Hepatitis A virus (HAV) and hepatitis B virus (HBV) are vaccine-preventable viral infections. Immunization for new admissions to Correctional Service Canada (CSC) represents an opportunity for public health intervention.

METHODS: While vaccine costs are standard across CSC, staff time and serology costs vary by region. Region-specific cost models of $90 \%$ coverage of new admissions at intake were built, comparing four immunization program scenarios across overall HBV immunity levels ([HBVp]) of 0.10-0.60: 1) Twinrix ${ }^{\mathrm{TM}}$ (vaccinate all inmates on admission regardless of serology status);

2) Twinrix ${ }^{\mathrm{TM}}$ with serology (vaccinate according to HBV immunity);

3) Combined Twinrix ${ }^{\mathrm{TM}} / \mathrm{Vaqta}^{\mathrm{TM}}$ regime based on HBV/HAV serology; and

4) Separate Engerix ${ }^{\mathrm{TM}} /$ Vaqta $^{\mathrm{TM}}$ regimes based on HAV/HBV serology.

RESULTS: Cost of scenario 1 was $\$ 799,361$ independent of [HBVp]. The cost for scenario 2 ranged from $\$ 1,018,475$ to $\$ 618,795$; parity with scenario 1 was at $[\mathrm{HBV} \mathrm{p}]=0.35-0.40$. The cost for scenario 3 ranged from $\$ 1,043,507$ to $\$ 685,545$; parity with scenario 1 was at $[\mathrm{HBV} \mathrm{p}]=0.45-0.50$. The cost for scenario 4 ranged from $\$ 997,028$ to $\$ 609,263$; parity with scenario 1 was at $[\mathrm{HBV}]=0.35-0.40$. Data from 888 newly admitted male inmates participating in a pilot program in 2004 indicate that $79 \%$ of inmates were screened for HBV. Overall, [HBVp] was estimated at 0.51 , with serological confirmation of vaccination accounting for half the observed HBV immunity.

CONCLUSION: As the population prevalence of HBV immunity increases, HBV serology becomes more cost effective. These data suggest that, given the estimated $[\mathrm{HBV}]$ among new admissions, an immunization program that includes HAV/HBV serology testing and appropriate vaccination would be cost effective. The increasing availability of community-based immunization programs for HBV in Canada suggest an increasing prevalence of HBV immunity in future cohorts of new admissions to CSC. A national electronic immunization registry would further reduce costs by validating self-reported vaccination histories.

\section{P63}

PHARMACISTS AS IMMUNIZERS: KEY DETERMINANTS OF COMMUNITY PHARMACISTS' WILLINGNESS TO ADMINISTER IMMUNIZATIONS TO ADULTS

E Corsten, Dalhousie University; S Bowles, School of Pharmacy, Dalhousie University; B Power, Canadian Pharmacists Association; S McNeil, Canadian Centre for Vaccinology, Halifax, Dalhousie University

INTRODUCTION: Underutilization of vaccines is a significant public health problem. Adult immunization rates in Canada fall below desired targets. Barriers to immunization include inadequate public education about vaccines, lack of access, and convenience issues. Pharmacists are highly accessible professionals who could potentially increase immunization rates among Canadian adults by administering vaccines. Most Canadian jurisdictions do not permit pharmacists to immunize; however, pharmacist immunizer models are successfully employed in other countries. We sought to determine Canadian pharmacists' knowledge, attitudes and beliefs about immunization, and to understand potential barriers to pharmacists acting as immunizers.

METHODS: An Internet-based survey was emailed to 4154 community pharmacists practicing in Canada in July 2006. The survey was piloted through focus groups for qualitative feedback and tested for content validity and test-retest reliability prior to dissemination. Data collection is ongoing.

RESULTS: By August 11, 2006, there were 390 responses to the survey (9.4\%). Responses were received from all provinces and territories. $43 \%$ were pharmacy owners or managers. The majority (96\%) agreed that increasing immunization coverage rates is important and that pharmacists as immunizers would increase public access $(88 \%)$, improve coverage rates $(84 \%)$, and be acceptable to the public $(72 \%)$. However, only $67 \%$ agreed that pharmacists should be permitted to immunize; $50 \%$ said they are willing to incorporate immunization into their practice if permitted, and $23 \%$ remained unsure. Only $17 \%$ described their current training as adequate, $97 \%$ agreed more professional training would be required to administer immunizations safely, and $90 \%$ agreed that certification in vaccine administration should be required. Pharmacists identified liability, reimbursement, and negative interactions with other providers as important barriers to pharmacists administering vaccines.

CONCLUSION: Pharmacists could be important resources to increase adult vaccine coverage rates in Canada. Most are willing to expand their practice scope to include immunization. However, implementation of any practice change would require professional development and certification in vaccine administration. Policy will need to be informed by issues such as reimbursement and liability.

\section{P64}

WHAT'S PUBLIC? WHAT'S PRIVATE? POLICY TRADE-OFFS AND THE DEBATE OVER MANDATORY INFLUENZA VACCINE FOR HEALTH CARE WORKERS

C Mah, University of Toronto

INTRODUCTION: In May 2000, the Ontario government amended its provincial Ambulance Act, including a change to communicable disease standards requiring mandatory annual influenza vaccination for all ambulance personnel. Ambulance workers launched a section 7 Charter of Rights suit against the province in 2001. Public furor escalated, with job suspensions for personnel who refused to comply. The province ultimately revised standards in October 2002 to promote, rather than mandate, the vaccine. Governments and policymakers remain reticent to endorse mandatory vaccination. Given pandemic preparedness scenarios, clarifying the dialogue around mandatory influenza vaccination for health care workers has key policy implications.

METHODS: This policy analysis uses document analysis. Material analyzed includes clinical literature, practice guidelines, surveys, legislation, case law, news coverage, and interest group publications.

ANALYSIS: Policy decisions about personal health services differ from those for public health services. Decisions on who will approve, pay for, and deliver services are accompanied by decisions on when and how to 
compel individual behaviour. As Deborah Stone has observed, trade-offs must be made between such policy goals as liberty, security, efficiency, and equity. Policy becomes complex because different stakeholders interpret evidence differently. Stakeholders assign different weights to policy goals and may even define the same goals differently. Proponents as well as opponents of mandatory vaccination, for example, convey their arguments in security terms; proponents emphasize sub-clinical infections among workers and duty of care (public security) while opponents emphasize risk of adverse events (personal security). Proponents assert less worker absenteeism (efficiency) while opponents stress coercion and alternate personal infection control measures (liberty and individual rights/responsibilities). Consequently, stakeholders talk past each other. Determining the place of mandatory influenza vaccination for health care workers thus demands reconciling policy contradictions and clarifying the underlying disputes hidden in the language of the mandatory vaccination debate.

\section{P65}

KNOWLEDGE, ATTITUDES AND BARRIERS TO CHILDHOOD VACCINATIONS IN ON-RESERVE FIRST NATIONS COMMUNITIES IN SOUTHERN ALBERTA

R Hering, University of Calgary; WR Yacoub, First Nations Inuit Health Branch - Alberta Branch- Health Canada; R Richardson, First Nations Inuit Health Branch - Alberta Branch- Health Canada; JD Kellner, Alberta Children's Hospital - University of Calgary INTRODUCTION AND RATIONALE: In many First Nations (FN) communities in Canada, childhood vaccination $(\mathrm{CV})$ rates are lower than in comparable populations and the incidence of vaccine-preventable infections is higher. We studied current knowledge, attitudes, and barriers towards $\mathrm{CV}$ s in FN communities.

METHODS: A survey was given to consenting parents $(\mathrm{P})$ and health providers (HP) during investigator visits to $6 \mathrm{FN}$ communities in southern Alberta in 2006.

RESULTS: $160 \mathrm{P}$ and $48 \mathrm{HP}$ completed the survey. Overall, $93 \%$ of $\mathrm{P}$ believed that CVs are important and $84 \%$ believed them to be safe. However, $25 \%$ thought that $\mathrm{CV}$ s can give you the disease you are trying to avoid while $60 \%$ believed that children should get sick much less, if at all, after getting their CVs. Only $27 \%$ of HP and 30\% of P thought that children should get vaccinated when they have a minor illness such as a cold. HP's beliefs about what P believe differed from P's beliefs in some respects: $57 \%$ of HP thought $\mathrm{P}$ were concerned about needles hurting children vs. $8 \%$ of $\mathrm{P}(\mathrm{P}<0.001) ; 35 \%$ of HP thought that traditional beliefs made $\mathrm{P}$ less likely to get their children vaccinated vs. $10 \%$ of $\mathrm{P}(\mathrm{P}<0.001)$; and $63 \%$ of HP thought $\mathrm{P}$ were concerned about vaccine side effects vs. $19 \%$ of $\mathrm{P}$ $(\mathrm{P}<0.001)$. Barriers to $\mathrm{CV}$ s for $\mathrm{P}$ included getting time off work $(35 \%)$, and remembering when their child needed vaccinations (34\%). P who reported that their children had been fully vaccinated on time were less likely to be concerned about their children getting 3 or more needles at one time $(\mathrm{P}=0.006)$ and were less likely to say that their traditional beliefs discouraged them from vaccinating their children. $(\mathrm{P}=0.02)$.

CONCLUSIONS: Parents generally support CVs, however practical barriers reduce levels of vaccination. Although mostly accurate, health providers have some misconceptions about parental CV beliefs.

\section{P66}

EPIDEMIOLOGICAL IMPACT AND VACCINE

EFFECTIVENESS FOLLOWING A HIGH SCHOOL PERTUSSIS VACCINATION PROGRAM IN NEW SOUTH WALES, AUSTRALIA

C Rank, The University of Sydney

INTRODUCTION: Several countries have introduced adolescent/adult formulation diphtheria-tetanus-pertussis (dTap) vaccines in recognition of an increased incidence of pertussis among older individuals. A three component dTap vaccine (Boostrix ${ }^{\mathrm{TM}}$ ) was added to the Australian immunization schedule in 2003. To respond to continued epidemics in a susceptible cohort of adolescents in New South Wales (NSW), a dTap vaccination program was carried out in 2004 targeting all high school students. This was the first large scale use of the vaccine in Australia, and since its introduction there has been limited data to evaluate the program. This study aims to describe the impact of adolescent pertussis vaccination on the incidence of disease, and estimates the effectiveness of Boostrix ${ }^{\mathrm{TM}}$ in the field.

METHODS: Notification data between January 1999 and December 2005 were analyzed, comparing the incidence and proportion of pertussis cases by age before and after the program. The vaccination status of all 2005 adolescent pertussis cases in NSW was obtained from follow up of the high school vaccination program records. Vaccine effectiveness (VE) was calculated by logistic regression using the screening method.

RESULTS: The incidence of pertussis in adolescents aged 10-19 was 2.5 times greater (95\% CI: 2.3-2.8) during the pre-program period 1999-2003 than in 2005. The proportion of all pertussis cases comprised by this age group was lowest in $2005(6.6 \%)$ compared to previous years, whilst the percentage of cases among adults over 20 years of age rose from $55.5 \%$ in 2003 to $87.2 \%$ in 2005 . Vaccine effectiveness when adjusted for health area and diagnostic method was $78.0 \%$ (95 \% CI: 60.7 to $87.6 \%$ ).

CONCLUSIONS: The NSW dTap vaccination program provides preliminary evidence that booster vaccination can effectively control pertussis in adolescents. Continued surveillance is important as successive adolescent cohorts are vaccinated. The screening method provides an opportunity to monitor VE under field conditions and can complement the routine surveillance of pertussis.

\section{P67}

\section{THE VAXED PROJECT: A CANADA-WIDE CURRICULUM REVIEW AND ASSESSMENT OF VACCINE KNOWLEDGE AND ATTITUDES AMONG TRAINEES IN HEALTH PROFESSIONAL PROGRAMS}

L Pelly, Canadian Centre for Vaccinology; BA Halperin, Canadian Centre for Vaccinology, Halifax; D Baxendale, Canadian Centre for Vaccinology, Halifax; D Pierrynowski-Gallant, St Francis Xavier School of Nursing; S Bowles, Dalhousie University College of Pharmacy; N Macdonald, Canadian Centre for Vaccinology, Halifax; R Strang, Nova Scotia Department of Health; K Mann, Faculty of Medicine, Dalhousie University; SA McNeil, Canadian Centre for Vaccinology, Halifax

INTRODUCTION: Knowledge and attitudes of healthcare providers (HCP) have significant impact on frequency with which vaccines are offered and accepted but many HCP are ill equipped to make informed recommendations about vaccine merits and risks. We performed a comprehensive assessment of trainee needs regarding immunization, to be used to develop a multi-faceted, evaluable, interprofessional educational intervention. METHODS: (i) A 5-part questionnaire was sent to all Canadian nursing, medical and pharmacy schools to assess immunization-related curriculum content; information was elaborated by phone interview; (ii) A 77-item web-based, validated questionnaire was emailed to final-year students in medicine, nursing, and pharmacy at Dalhousie University and St Francis Xavier University School of Nursing to assess knowledge, attitudes, and behaviors reflecting current immunization curriculum.

RESULTS: The curriculum review yielded responses from 18\%, 48\%, and $56 \%$ of medical, nursing, and pharmacy schools, respectively. Time spent on immunization content varied substantially between and within disciplines from $<1$ to $>50 \mathrm{hrs}$. Most schools reported some content regarding vaccine preventable diseases, immunization practice and clinical skills but there was considerable variability and fewer schools had learning objectives or formal testing in these areas. Gaps in knowledge of vaccine indications/contraindications and safety were identified in all programs (mean correct $=13.5 / 21) ; 33.4 \%$ of trainees were unsure if there is a relationship between pertussis vaccine and sudden infant death syndrome and $22.3 \%$ were unsure whether current evidence supports a link between vaccines and chronic diseases. 74\% of respondents don't feel comfortable discussing vaccine side effects with parents/patients and only $21 \%$ felt they received adequate teaching regarding immunization during training.

CONCLUSIONS: Important gaps were identified in knowledge of graduating nursing, medical, and pharmacy trainees regarding vaccine indications/contraindications, adverse events and safety. The national curriculum review revealed wide variability in immunization curriculum 
content and evaluation. The results suggest that all HCP trainees could benefit from development of core objectives for immunization providers and comprehensive, interprofessional education strategies to achieve these objectives during training.

\section{P68}

\section{IDENTIFYING BEST PRACTICES FOR COLD CHAIN} MAINTENANCE: A PUBLIC-PRIVATE PARTNERSHIP C McIntyre, British Columbia Centre for Disease Control (BCCDC); E Galanis, BCCDC; J Rousseau, sanofi pasteur; D Easterbrook, Cryopak Industries Inc

OBJECTIVE: To identify best practices for cold chain maintenance during mass immunization clinics.

METHODS: A survey was completed by British Columbia public health staff to assess current equipment used, and vaccine transport and handling practices for cold chain maintenance for immunization clinics. On-site visits enabled industry partners to observe practices during clinics. Coolers chosen by public health were tested under laboratory conditions, re-enacting actual environmental patterns and current practice needs. Education sessions were held to describe testing results, recommendations for equipment to use, and best practices to maintain the cold chain during immunization clinics.

RESULTS: Survey response rate was $59 \%$. In $20 \%$ of jurisdictions, vaccines may be in the field for more than 8 hours. $68 \%$ of coolers are not packaged differently in summer and winter despite temperature differences. $46 \%$ open coolers over 10 times during a clinic and 7\% keep coolers open for over a minute each time. All clinics observed used large vaccine carriers and transferred vaccines to smaller coolers at vaccination stations. Cooler testing indicated that coolers packed with flexible insulating blankets surrounding the vaccine mass, and use of both frozen and refrigerator-temperature gel packs, would maintain the vaccines at the desired temperature for extended periods of time. Specific packing configurations were recommended for summer and winter conditions. With temperatures above $38^{\circ} \mathrm{C}$, replacing gel packs and flexible insulating blankets after a specified period of time would provide continued protection of vaccines.

CONCLUSIONS: Vaccines can be protected during the extended transport and clinic times that occur with mass immunization clinics. Based on laboratory testing, $\mathrm{BC}$ public health staff have recommendations for equipment and packing procedures for cold chain maintenance. This is an example of public health and industry partnership which will enhance preparedness for infectious disease events requiring mass immunization.

\section{P69}

\section{A REVIEW OF HERD EFFECTS IN THE ECONOMIC EVALUATION OF THE 7-VALENT PNEUMOCOCCAL CONJUGATE VACCINE}

C Vicente, Wyeth; JD Kellner, Department of Pediatrics, University of Calgary, Calgary, Alberta; P Jacobs, Institute of Health Economics, Edmonton, Alberta; D Han, Wyeth Pharmaceuticals, Markham, Ontario

OBJECTIVES: Many childhood vaccines not only protect those directly immunized, but also indirectly prevent infection or disease in the nonimmunized population through herd effects. Our objective was to review the methodological approaches of including the indirect outcomes of herd effects in economic evaluations of the 7-valent pneumococcal conjugate vaccine, Prevnar ${ }^{\circledR},($ PCV-7).

METHODS: An extensive systematic review of the literature was conducted. The EMBASE, MEDLINE, Biosis and Current Contents databases were reviewed from 1996-2006. Two independent researchers reviewed titles and abstracts of each article. Additional articles referenced in our primary search were reviewed for further inclusion. Articles meeting inclusion criteria were blinded. Two independent researchers then reviewed and extracted data from all included articles. The quality of each included article was assessed and any discrepancies were resolved through consensus. RESULTS: Our primary search resulted in 14 titles being identified. Five articles were original economic evaluations of PCV-7 that met our inclusion criteria and nine articles were excluded (all nine were reviews). Each economic evaluation was highly sensitive to variations in the estimates of herd effects. In reviewing how herd effects were being captured, various considerations were noted: 1 ) the source of herd effects and the generalizability from one population to another; 2) the association between herd effects and PCV-7 serotype coverage; and 3) implications of a static or dynamic approach to incorporate indirect benefits.

CONCLUSION: The cost-effectiveness of PCV-7 is highly sensitive to variations in herd effects. Improving the precision of measured outcomes attributed to herd effects in future economic evaluations will advance research in this field and assist in health policy decision-making.

\section{P70}

\section{USE OF A WEB-BASED LEARNING ENVIRONMENT TO IMPROVE ADACEL ${ }^{\circledR}$ UTILIZATION IN NOVA SCOTIA EMERGENCY DEPARTMENTS}

A Murphy, Dalhousie University; J Curran, IWK Health Centre; M Farhangmehr, Public Health Agency of Canada; S Best, IWK Health Centre; D Sinclair, Dalhousie University

INTRODUCTION: Emergency department (ED) practitioners continuously manage large quantities of new clinical information. This process is challenged by patient care issues (eg, volume, acuity) and staffing considerations (eg, scheduling). Deficiencies in ED clinician information management strategies and knowledge regarding vaccines results in inappropriate utilization. A needs assessment of Nova Scotia ED clinicians identified vaccines as a topic for continuing education (CE). The purpose of this research was to determine the utilization of Adacel ${ }^{\circledR}$ in Nova Scotia EDs and the efficacy of a web-based CE approach to improve knowledge and vaccine utilization.

METHODS: A pre-existing pediatric emergency, web-based CE learning environment was used. An Adacel ${ }^{\circledR} \mathrm{CE}$ module was developed and released to 3 cohorts of rural and urban EDs at prespecified times. Presentations with audio and video streaming included case-based scenarios demonstrating reciprocal reporting form completion instructions and appropriate vaccine use based on age, wound type, and immunization history. The module also contained a discussion board and pre and post-tests. EDs not receiving the CE served as the control group. Pre and post-tests and Adacel ${ }^{\circledR}$ reciprocal forms were collected and analyzed.

RESULTS: Of the 138 participants who entered the web-based environment at least once, 30 and 22 completed pre and post-tests, respectively. Post-test mean scores significantly improved (mean 1.86, 95\% CI 1.07, 2.65). 255 reciprocal forms were received. $47 \%$ of forms were appropriately completed with 50\% (89/178) and 51\% (19/37), from the cohort and control groups, respectively. $35 \%(90 / 255)$ of forms lacked sufficient information to determine appropriateness. Inappropriate vaccine use was $49 \%$ when combining inadequately completed forms (worst case scenario) and those deemed as inappropriate administration (36/255).

CONCLUSIONS: The web-based education improved knowledge for those who completed the module. Behaviour in practice demonstrated lack of adherence with form documentation and vaccine administration guidelines. Further research is needed to determine if web-based continuing education impacts provider behaviour.

\section{P71}

VALIDATION OF SENIORS' SELF-REPORTS OF INFLUENZA IMMUNIZATION

B Payne, Univerity of Manitoba; MK Hall, AIM Study, Community Health Sciences; V Menec, Community Health Sciences; L Guse, Nursing, University of Manitoba

INTRODUCTION: The validity of self-reports by seniors is often questioned perhaps due to the widespread belief of memory deficits in older adults. The objective of this study was to compare self-reports of influenza immunization by Manitoba Seniors with data from the province of Manitoba's immunization monitoring system (MIMS).

METHODS: For the self-reports, in-person interviews were conducted with 599 community dwelling, cognitively intact Manitobans (age: mean $=79$, range $=60-102$ ) in the summer of 2005 to document influenza 
immunization practice in the fall of 2004. The MIMS database records were derived from physician billing claims and from manual entry of public health provided immunizations for 2004. Sensitivity and specificity were calculated.

RESULTS: The MIMS database recorded that $71.5 \%$ of the study respondents had a flu shot in 2004, and $79.8 \%$ of respondents reported having a shot. MIMS had no entry for $28.5 \%$ compared to $20.2 \%$ for the self-reports of not having a flu shot. Sensitivity is $99 \%$ (self reported yes with a corresponding entry in MIMS). Specificity is $69 \%$ (self reported no and no entry in MIMS). No concordance in the MIMS data was evident for $8.8 \%$ who reported having a flu shot the previous year.

CONCLUSIONS: Overall, the concordance between the self-reports and the MIMS was very high suggesting that self-reports of influenza immunization by older adults are generally quite accurate. Possible explanations for uncorroborated positive reports $(8.8 \%)$ are: 1) Seniors may have remembered their intention to be immunized rather than the actual event; 2) Having been told that the interview would be about "flu shots", social desirability may have influenced responses; 3) Recall from close to one year before may have been incorrect; 4) Physician billing claims and manually entered public health records are not complete.

\section{P72}

\section{DETERMINANTS OF TWO IMMUNIZATION BEHAVIOURS OF BC IMMUNIZATION PROVIDERS}

K Pielak, BC Centre for Disease Control; V Remple, BC Centre for Disease Control; C McIntyre, BC Centre for Disease Control; J Buxton, BC Centre for Disease Control; B Halperin, Clinical Trials Research Center; D Skowronski, BC Centre for Disease Control INTRODUCTION: Due to growing concern about immunization coverage levels of two year olds in $\mathrm{BC}$, there was a recognized need to assess immunization provider behaviours that may hinder or promote immunization uptake. The purpose of this study was to ascertain the behavioural determinants and proportion of immunization providers who administer multiple injections at one client visit and who promote immunization at each client encounter (ie, avoid missed opportunities for immunization). METHODS: A cross-sectional postal survey based on the Theory of Planned Behaviour was sent to all immunization providers in BC. The survey elicited data on demographics, practice characteristics, and attitudes, perceived social norms and perceived behavioural control related to the two immunization behaviours. High behaviour was defined as self-reported performance of the behaviour at least $75 \%$ of the time. Logistic regression modeled the association between high behaviour and variables of interest and those achieving a statistical significance of at least 0.1 .

RESULTS: The survey was completed by 335 nurses and 343 physicians ( $67 \%$ and $22 \%$ response rate, respectively). $87 \%$ and $39 \%$ reported high behaviour for administration of multiple injections and promotion of immunization at each encounter, respectively. High behaviour for administration of multiple injections was associated with nursing profession (OR 8.5, 95\% CI 2.5-29.1) and providing $>10$ paediatric immunizations per month (OR 2.6, 95\%CI 1.4-4.9). High behaviour for promotion of immunization was associated with high perceived social norms ([OR 5.8, 95\%CI 3.8-8.9], [feeling they should offer any needed immunizations]) and high perceived behavioural control ([OR 2.1, 95\%CI 1.5-2.9], [feeling it takes too much time to offer all immunizations and that access to records is a barrier]).

CONCLUSIONS: The results will inform development of behavioural change interventions targeted at these determinants of immunization provider behaviour. With regard to the behaviour of offering immunization at each client encounter, there will be the challenge of identifying interventions that may influence the immunization provider's desire to comply with the opinions of their colleagues and clients, as well as the negative perception regarding the increased amount of time needed to offer all needed immunizations.

\section{P73}

DEVELOPMENT OF A SURVEY INSTRUMENT TO MEASURE

PSYCHOSOCIAL DETERMINANTS OF IMMUNIZATION INTENTIONS OF CANADIAN IMMUNIZATION PROVIDERS

K Pielak, BC Centre for Disease Control; C McIntyre, BC Centre for Disease Control; B Halperin, Clinical Trials Research Center, IWK Health Centre; J Buxton, BC Centre for Disease Control; V Remple, BC Centre for Disease Control; D Skowronski, BC Centre for Disease Control

INTRODUCTION: Survey instruments used to assess the motivators and barriers to immunization practices are generally not based on theoretical frameworks that enable results to inform behavioural change interventions. The project's objective was to develop and validate a research survey instrument that measures factors associated with immunization providers' (IP) intentions regarding key immunization practices based on the Theory of Planned Behaviour (TPB). The final instrument will be used for a national survey with the ultimate goal of informing the development of targeted IP education programs.

METHODS: An elicitation survey was conducted with 125 IP to determine what key behaviours (domains) were viewed as most critical to an optimal immunization delivery program. Thematic analysis produced nine domains and specific items measuring intentions, attitudes, subjective norms, and perceived behavioural control related to each domain. Content validity was evaluated by an expert panel of IP calculating a content validity index $(\mathrm{CVI})$ for each item and domain. Only those domains and items with statistically significant $(p<0.05)$ CVI were retained. Field testing informed further refinement.

RESULTS: The current instrument is comprised of 91 items assessing seven domains: (i) multiple injection for infants; (ii) annual receipt of influenza vaccination; (iii) offering immunization at each client encounter; (iv) pediatric MMR administration; (v) adverse reaction reporting; (vi) benefit-risk communication; and (vii) immunization of clients with minor illnesses.

CONCLUSIONS: Using the TPB, we have developed a survey instrument that will inform development of behavioural change interventions targeted at one or more of the psychosocial determinants of IP behavioural intention. The survey has been conducted in British Columbia and Nova Scotia. Several other Canadian provinces and territories are considering the use of the instrument to inform a national perspective.

\section{P74}

\section{CAN WE REACH OUR GOAL OF 90\% COVERAGE OF INFLUENZA VACCINATION UPTAKE FOR HEALTH CARE WORKERS?}

V Willaeys, University of British Columbia; P Daly, Vancouver Coastal Health; R Gustafson, Vancouver Coastal Health

INTRODUCTION: The goal of Vancouver Coastal Health (VCH) is to obtain an annual influenza vaccination coverage rate of $90 \%$ for health care workers (HCW). This has been achieved in 2005 in some non-VCH owned and operated residential care facilities. The coverage of regular employees of acute care facilities ranged between $23 \%$ and $69 \%$ with an average of $48 \%$.

METHODS: As part of a quality improvement project we conducted 15 semi structured interviews with clinical managers of health care workers in acute care, residential care, and community health facilities.

FINDINGS: The highest vaccination rates were obtained in Units where the clinical managers were dedicated to achieve high coverage. Experiencing an influenza outbreak or foreseeing the impact of low immunization rates during an influenza outbreak were identified as strong motivators. Other characteristics of high uptake Units were a personal follow up of unvaccinated health care workers, and many vaccination opportunities through clinics next to cafeteria, by appointment, by mobile units and by an on-site vaccinator. Concise factual information geared towards health care workers should be available addressing their major concerns. In Vancouver, with a high number of immigrants, posters in the most prevalent languages should be developed with information for health care workers with limited knowledge of English and visiting family members. 
CONCLUSIONS: To reach 90\% influenza vaccination uptake of HCW requires an investment of time, energy and financial resources. A comprehensive and site-specific approach is needed, involving patient/resident service managers, health care workers, senior management, occupational health, public health, nurse educators, infection control, and media.

\section{P75 \\ ARE WE USING EPINEPHRINE INAPPROPRIATELY FOR MILD ANAPHYLAXIS?}

V Willaeys, Universtity of British Columbia; P Daly, Vancouver Coastal Health; R Gustafson, Vancouver Coastal Health

INTRODUCTION: The use of epinephrine is the cornerstone of the treatment of anaphylaxis and can be lifesaving in case of an anaphylactic shock after immunization. But the inappropriate use of epinephrine may prevent the administration of future vaccines, may add to the distress caused by the adverse event, may cause drug interactions, and side effects which may be severe in the elderly with co-morbid conditions. After reports of inappropriate use of epinephrine in Vancouver Coastal Health for adverse events that were not anaphylaxis following immunization, we undertook a review of our anaphylaxis policy.

METHODS: An environmental scan of international anaphylaxis policies and a literature search for scientific evidence were performed.

FINDINGS: There is no universally accepted definition of "anaphylaxis". Clinical criteria to guide anaphylaxis management differ from country to country. In North America, epinephrine is given for mild and early anaphylaxis. In Europe, early treatment of anaphylactic shock is emphasized with watchful waiting for signs of early anaphylaxis. France recommends using epinephrine urgently in case of respiratory distress, hypotension, generalized urticaria and antecedents of a serious anaphylactic reaction. The Australian guideline advises the use of adrenaline by intramuscular injection for any signs of anaphylaxis, except for erythema (flushing) or itching alone, which are observed for progression. The evidence base supporting the best approach is limited.

CONCLUSION: The experience of Australia and European countries suggests that the inclusion in the guidelines of a recommendation to observe closely for mild, non specific skin reactions such as erythema, itchiness or local reaction at injection site, and watchful waiting for the occurrence of early respiratory or cardiovascular changes may prevent inappropriate use of epinephrine without evidence of causing a more adverse outcome.

\section{P76 \\ HUMAN PAPILLOMAVIRUS VACCINE DECISION MAKING IN CANADA}

S Deeks, Public Health Agency of Canada (PHAC); M Landry, Ministère de la Santé et des Services sociaux du Québec; S Dobson, Vaccine Evaluation Center, University of British Columbia; M Farhangmehr, Immunization \& Respiratory Infections Division, PHAC; M Lalonde, Immunization \& Respiratory Infections Division, PHAC; T Tam, Immunization \& Respiratory Infections Division, PHAC

BACKGROUND: Guardasil ${ }^{\mathrm{TM}}$, a quadrivalent Human Papillomavirus (HPV) vaccine was approved for use in Canada on July 10, 2006. Although vaccine recommendations are made at the national level, each province and territory is responsible for funding and determining when and how publicly funded immunization programs are introduced in their jurisdiction. This results in variation in program delivery across the country. PROCESS: A multi-disciplinary HPV Vaccine Workgroup has been established to develop comprehensive recommendations for vaccine use in Canada, using an analytic framework for immunization programs (1). It includes members from the National Advisory Committee on Immunization and the Canadian Immunization Committee (federal, provincial and territorial programs), as well as disease experts. The Canadian Cancer Prevention and Control Network, College of Family Physicians of Canada, Society of Obstetricians and Gynaecologists of Canada, and First Nations and Inuit Health are represented. Broad representation from public health, vaccinology, sexual health, gynecology, cancer, aboriginal health, nursing and family medicine ensures that the workgroup's mandate is met. The components of the framework that will be examined include: disease characteristics and burden, vaccine characteristics, alternate immunization strategies, social and economic costs and benefits, feasibility and acceptability, ability to evaluate programs and research questions. The recommendations will be scientific and programmatic in nature, taking into consideration the Canadian health care system. To date, there have been 3 meetings of the workgroup and HPV vaccine program options have been identified and prioritized. Recommendations are expected by year end.

CONCLUSION: This is the first time the analytical framework will be used to provide justification for the introduction of a vaccine nationally. It is anticipated that this process will result in a more harmonized implementation of HPV vaccine programs across the country. Reference:

1. Erickson LJ, De Wals P, Farand L. An analytical framework for immunization programs in Canada. Vaccine 2005;23:2470-2476.

\section{P77}

THE COST OF HEPATITIS A IN CANADA: MINISTRY AND SOCIETAL PERSPECTIVES

A Anonychuk, University Health Network, Toronto, Ontario; M Krahn, University Health Network, Toronto, Ontario; V Gilca, Universite Laval, Beauport, Quebec; C Bauch, University of Guelph, Guelph, Ontario; B Pham, University of Toronto, Toronto, Ontario; B Duval, Universite Laval, Beauport, Quebec

INTRODUCTION: Even though Hepatitis A (HA) cases are vaccinepreventable, an average 434 cases $(1.4 / 100,000)$ in 2000-2004 were reported annually in Canada. As consolidated data is lacking, the objective of this study was to estimate the economic burden of HA in Canada.

METHODS: A comprehensive literature search (eg, Medline January 1966-March 2005 and Embase January 1980-March 2005) was conducted to identify data on resources utilization and costing of HA infection. Unpublished data were identified through the Ontario Case Costing Initiative, Statistics Canada and provincial Ministries of Health, among others. Remaining data gaps were solicited through expert panel. Ministry perspective included direct infection costs (hospitalization, physician visits, etc). Societal perspective included direct costs plus time costs (cost of lost productivity). All costs were expressed in 2005 Canadian dollars.

RESULTS: Approximately $67 \%$ of symptomatic HA cases were reported to public health. The average direct cost from a ministry perspective was $\$ 1,449$ and $\$ 1,905$ per symptomatic and reported case, respectively. Approximately half of the former cost was attributed to hospitalization (48\%), physician visits (26\%), public health costs $(23 \%)$, and liver transplant and death (1\% each). The average cost per symptomatic case was $\$ 2,889$ when time costs were included. The highest cost was in adults aged $>30$ years $(\$ 4,300)$. In pre-vaccination era $(1988-1994)$, the annual cost of reported cases was approximately $\$ 3.4 \mathrm{M}$ and $\$ 5.6 \mathrm{M}$ from ministry and societal perspective, respectively. The corresponding estimates for the period of $1995-2003$ were $\$ 2.3 \mathrm{M}$ and $\$ 4.1 \mathrm{M}$, respectively. The age group incurring the largest societal cost was 30-59, with approximately $50 \%$ and $62 \%$ of the total ministry and societal cost, respectively for 1995-2003.

CONCLUSION: HA remains a significant public health and economic burden, despite the decrease in the reported incidence. The data in this study may help in the evaluation of cost-effectiveness of future HA vaccination programs in Canada. 
P78

COÛTS ET EFFICACITÉ DU PROGRAMME DE

VACCINATION DES ENFANTS DE 0-2 ANS AU QUÉBEC

M Guay, Université de Sherbrooke; M Blackburn, Direction de santé publique (DSP) Montérégie, Longueuil, Québec; P Clément, Institut national de santé publique du Québec, Montréal, Québec; A Tremblay, DSP Montérégie, Longueuil, Québec; C St-Hilaire, AÉTMIS, Montréal, Québec; AM Clouâtre, DSP Montérégie, Longueuil, Québec; L Rousseau, DSP Laval, Laval, Québec et Université de Montréal; $M$ Landry, Ministère de la santé et des services sociaux du Québec (MSSS); A Pelletier, Institut national de santé publique du Québec, Montréal, Québec; M Dionne, Institut national de santé publique du Québec, Québec, Québec; D St-Amand, AQES, Montréal, Québec

OBJECTIFS: 1) Évaluer les coûts et l'efficacité du programme de vaccination québécois des 0-2 ans. 2) Comparer les coûts selon le lieu de vaccination, soit en Centre local de services communautaires (CLSC) ou en clinique médicale (CM).

MÉTHODE: Devis quantitatif et qualitatif selon une perspective sociétale (ministère de la Santé et des Services sociaux (MSSS), direction de santé publique (DSP), CLSC, médecins vaccinateurs et parents considérés). L'approche de micro-costing a utilisé 14 sources de données (collecte réalisée en 2004-2005). L'évaluation des coûts porte sur dix territoires de CLSC sélectionnés selon leurs caractéristiques sociodémographiques et celles des services d'immunisation. Des estimations selon la perspective des acteurs sont établies pour des coûts totaux annuels (CTA), des coûts unitaires par visite (CPV) et des coûts par dose (CPD). Selon la perspective sociétale, un ratio coût-efficacité (efficacité évaluée par proxy: couverture vaccinale méningocoque) et une analyse de minimisation des coûts des CPV et CPD sont présentés.

RÉSULTATS: Le CTA assumé par le MSSS est de 22 millions \$. Les CTA assumés par les DSP varient de 31321 \$à 352805 \$. Le CPV assumés par les CLSC est de 36 , mais le CPD de 18 \$. Le CPV assumé en CM est de 7 \$. Le CPV assumé par les parents est de 18 \$. La couverture vaccinale s'élève à $86 \%$. Le ratio coût-efficacité est de 150 \$ par visite pour chaque point de pourcentage de couverture vaccinale obtenu. En retirant les déboursés des vaccins, le CPV en CLSC est de 50 \$, mais de 36 en CM. La vaccination en CM est moins coûteuse dans l'analyse de minimisation des coûts et dans l'analyse de sensibilité des CPV et CPD.

CONCLUSION: La vaccination des enfants génère des coûts élevés. Pour assurer des services de vaccination adéquats, les résultats de l'étude pourront être utilisés afin de revoir le financement des services pour qu'il corresponde aux coûts réels, autant en CLSC qu'en CM.

\section{POSTER PRESENTATIONS Viral Diseases and Vaccines}

\section{P79}

\section{PATTERNS OF HEALTH CARE RESOURCES UTILIZATION ASSOCIATED WITH CERVICAL CANCER SCREENING}

A Demers, CancerCare Manitoba; EV Kliewer, CancerCare Manitoba; L Elliot, University of Manitoba; R Lotocky, CancerCare Manitoba; B Shearer-Hood, CancerCare Manitoba; K Decker, CancerCare Manitoba; M Brisson, Merck Frosst

BACKGROUND: The impact of the new HPV vaccine on patterns of screening for cervical cancer and the associated use of health care resources is hard to predict and will depend on the response of public institutions and awareness of physicians. To estimate and assess its impact, it is necessary to have baseline information.

OBJECTIVE: To determine the current trends in health care resources utilization related to cervical cancer screening.

METHOD: Information was abstracted from population-based databases in Manitoba, Canada for the period 1984 to 2004 including the Cervical Screening Registry, Physician Claims, Hospital Separations, Manitoba Population Registry and the Manitoba Cancer Registry. Age-specific and age-standardized incidence trends of medical services utilization were determined.
RESULTS: The pattern of screening services and associated procedures provided to and/or used by women dramatically changed over the 20-year survey period. Rates of Pap (Papanicolaou) tests have decreased $11 \%$ (36/100 women in 1985, 32/100 in 2004), mainly due to the lower utilization in women 15 to 29 years of age. Rates of colposcopy have increased $150 \%(0.96 / 100$ in $1985,2.4 / 100$ in 2004) and rates of cone surgery have increased $346 \%(26.9 / 100,000$ in $1985,120.1 / 100,000$ in 2004), with the largest increase seen in women 20 to 39 years of age. Results on trends of lesion destruction (any method), biopsy and cryosurgery, as well as recent incidence rates of LSIL and HSIL (2002 to 2005), will also be presented. CONCLUSION: Patterns of cervical cancer screening and associated procedures showed important changes over the study period. The dynamic patterns of health services utilization will make long-term prediction of the impact of the vaccine challenging.

Study funded by Merck Frosst Canada

\section{P80}

\section{CHARACTERIZATION OF THE MONOCLONAL ANTIBODIES USED TO DEFINE THE IMMUNOGENICITY GARDASIL $^{\mathrm{TM}}$, A VACCINE AGAINST HUMAN PAPILLOMAVIRUS}

J Mansi, Merck Frosst Canada Ltd; R Kowalski; M Esser; M Brown; J Bryan

BACKGROUND: Gardasil ${ }^{\mathrm{TM}}$ is a prophylactic vaccine against four common human papillomavirus (HPV) types 6, 11, 16 and 18 . The vaccine consists of multiple copies of the L1 protein which self-assemble to form virus-like particles (VLP). The VLPs display a variety of epitopes. Some epitopes are shared between types while others are conformational and type-specific. A set of $\mathrm{mAbs}$ were evaluated for their neutralizing ability in a pseudo-neutralization assay and by hemagglutination inhibition (HAI). The antibodies were employed in a competitive immunoassay to demonstrate HPV type-specific seroconversion upon vaccination and to provide a link to neutralization.

METHODS: Eight mAbs, two per vaccine type were evaluated by ELISA and Luminex-based immuno assays (LIA) for conformational binding specificity with respect to HPV type. The mAbs were assessed for the ability to inhibit hemagglutination (HAI) as a surrogate for prevention of cell surface binding by the corresponding native virion. Lastly, the mAbs were evaluated in pseudo-neutralization assays for their ability to neutralize pseudovirus (PsV) infection of cells grown in culture. A competitive LIA (cLIA) using the best neutralizing mAbs identified for each type was performed on sera of GARDASIL ${ }^{\mathrm{TM}}$ vaccinated non-human primates to assess whether the vaccine induced neutralizing antibodies.

RESULTS: All eight mAbs were shown to bind conformational epitopes. At least one mAb for each HPV type was shown to be type specific and have neutralizing potential in the HAI and pseudo-neutralization assays. All vaccinated subjects seroconverted and demonstrated the presence of neutralizing antibodies.

CONCLUSIONS: Individually, H6.M48, K11.B2, H16.V5 and H18.J4 mAbs demonstrated the best neutralization potential for type 6, 11, 16 and 18 , respectively. These mAbs can be used effectively to evaluate Gardasil ${ }^{\mathrm{TM}}$ induced antibodies to neutralizing epitopes on the VLPs providing a link between seroconversion and protective immunity.

\section{P81}

GARDASIL ${ }^{\text {TM }}$ ANTIBODIES CROSS-NEUTRALIZE INFECTION OF VACCINE TYPE-RELATED HPV TYPES

\section{J Mansi, Merck Frosst Canada Ltd; M Brownlow; M Brown;}

M Esser; W Ruiz; J Bryan

Gardasil $^{\mathrm{TM}}$ is a quadrivalent HPV 6, 11, 16 and $18 \mathrm{~L} 1$ virus-like particle (VLP) prophylactic vaccine that functions by eliciting neutralizing antibodies which prevent viral infection of vaccine HPV types. The vaccine has been shown to be highly efficacious. We have evaluated the ability of serum antibodies from HPV VLP vaccinated women from clinical trials to cross-react and bind to VLPs of vaccine-related HPV types both within the A7 (HPV 18-related) and A9 (HPV16-related) species. In addition, utilizing the pseudovirion $(\mathrm{PsV})$ neutralization assay developed by the 
NCI, we evaluated the ability of serum antibodies elicited by Gardasi ${ }^{\mathrm{TM}}$ vaccination to neutralize HPV pseudovirus infection of vaccine-related HPV types. Efficacy protection studies are an indirect measure based on statistical assumptions of incidence of infection and the demonstration of a reduction in the projected incidence rate. The significance of this study is that it is a direct measure of vaccine-induced antibody protection against infection. In this study we demonstrate that Gardasil ${ }^{\mathrm{TM}}$ elicits antibodies which cross-react and bind to VLPs of vaccine-related HPV types (ie, A9 species 31, 52 and 58 and A7 species 45). In the pseudovirion neutralization assay, the vaccine was expected to protect against $\mathrm{PsV}$ infection of HPV types 16 and 18, which it did, however, we also demonstrated neutralization of vaccine-related PsV infection including HPV types 45 and 31. This study provides direct evidence, at the molecular level, that Gardasil $^{\mathrm{TM}}$ induces the production of antibodies that confer cross protection by demonstrating neutralization of infection of non-vaccine HPV types. Although the minimum antibody level required for protection has not yet been determined, the data are supportive of the ability of the vaccine to confer cross protection against vaccine-related HPV types, potentially expanding the prevention coverage against cervical cancer to impacting a greater percentage of the overall disease burden.

\section{P82}

\section{AGE-RELATED TRENDS IN THE TIMELINESS AND PREDICTIVENESS OF MEDICAL VISITS, HOSPITALIZATIONS AND DEATHS DUE TO PNEUMONIA AND INFLUENZA}

D Skowronski, BC Centre for Disease Control; M Chong, BC Centre for Disease Control; DM Skowronski, BC Centre for Disease Control; J Dhaliwal, BC Centre for Disease Control; J Brownstein, Harvard Medical School, Massachusetts, USA

INTRODUCTION: In a recent US study, medical visits for respiratory illness among children $<5$ yrs of age were the first and strongest predictors of overall peaks in mortality due to pneumonia and influenza (P\&I). Another US study instead emphasized the role of working-age adults. We explored similar age-related trends in British Columbia (BC), Canada. METHODS: Age-related associations were examined from medical service plan (MSP) billings, hospital discharge and vital statistics data for 1998-99 to 2003-04 in BC. Timeliness was the difference between the first peak in MSP influenza claims and first peak in hospitalization or death directly due to P\&I for each agegroup and cycle. Poisson regression was run per age group and cycle: dependent variable was hospitalization or mortality counts with prediction based on MSP claims for the period derived from timeliness analysis. Predictiveness was expressed as the average proportion of the deviance explained by age group.

RESULTS: Seasonal variation in the dominant influenza type/subtype, height and timing of peaks was identified. Highest MSP claim rates were in children $6-23 \mathrm{~m}, 2-4 \mathrm{yrs}$ and 5-9yrs $(1.8,1.7$ and 1.3/1000). Highest P\&I hospitalization rates were in children $<6 \mathrm{~m}, 6-23 \mathrm{~m}$ and the elderly $(0.5,0.5$ and $0.4 / 1000$ ), and highest death rates were in children $<6 \mathrm{~m}, 6-23 \mathrm{~m}$ and the elderly $(0.05,0.01$ and $0.1 / 1000)$. After adjustment for season, medical claims in children aged $6 \mathrm{~m}-4 \mathrm{yrs}$ and adults $20-49 \mathrm{yrs}$ were the strongest predictors of P\&I peaks in hospitalization ( $73 \%$ of the deviance each) and death ( $45 \%$ of the deviance each) with children aged $2-4 \mathrm{yrs}$ demonstrating earliest medical visits.

CONCLUSION: Young children and the elderly suffer the greatest direct effects from influenza and have the highest rates of related healthcare use. Young children, especially pre-school aged, and working-age adults may amplify the indirect risk to others through a combination of susceptibility and complex social networks facilitating transmission. Variation between studies in age-related findings may be due to differences in social structure and mixing patterns, health- or day-care access, diagnostic code validity and remuneration, and influenza seasons and strains. Administrative data should be used cautiously in guiding age-targeted recommendations. Region-specific analyses may be warranted.

\section{P83}

\section{IMMUNOGENICITY MEASUREMENTS OF THE HPV VACCINE, GARDASIL ${ }^{\circledR}$}

J Mansi, Merck Frosst Canada Ltd; M Esser; J Bryan

BACKGROUND: Gardasi ${ }^{\circledR}$ is a prophylactic Human papillomavirus (HPV) type 6, 11, 16 and 18 vaccine. There is no standard HPV serology assay. A useful immunoassay would show antibody-levels as an important correlate to vaccine-induced protection against infection. Here we describe the merits of a competitive Luminex immunoassay (cLIA) verses a total IgG LIA.

METHODS: Sera from 10 Gardasil $^{\circledR}$ vaccinated subjects were tested. Luminex beads with unique dye spectra for each HPV type were coated with the corresponding HPV type virus-like particles (VLPs). Total endpoint dilution LIA assays1 were performed using a secondary anti-human IgG antibody across five-fold dilutions. A cLIA assay 2 was also tested utilizing neutralizing HPV 6, 11, 16 and 18 conformational, type-specific mAbs.

RESULTS: Both immunoassays indicated seroconversion with seropositive titers boosted after each dose. The titres plateaued and remained steady month 12 through month 48 . The total IgG titers elicited by each HPV type were comparable to one another. The results of the four cLIAs are independent of one another, but demonstrate the presence of neutralizing antibodies to the specific HPV VLP types.

CONCLUSIONS: The total IgG LIA assessed seroconversion and comparisons were made between seropositive titres for the different HPV types within the vaccine. The cLIA measured only a portion of the antibodies elicited by the vaccine, however, the presence of these neutralizing antibodies suggests cLIA provides a measurement of vaccine induced protective antibodies. Thus both assays have merit, however the cLIA provides more clinically relevant data to support the efficacy of the humoral response to all four VLP in Gardasil ${ }^{\circledR}$.

References:

1. Ruiz W, McClements WL, Jansen JU, Esser MT. Kinetics and isotype profile of antibody responses. J Imm Based Ther Vaccines 2005;3:2.

2. Dias D, VanDoren J, Schlottmann S, et al. Optimization and validation of a multiplexed Luminex assay to quantify antibodies to neutralizing epitopes on human papillomaviruses 6, 11, 16 and 18. Clin Diagn Lab Immunol 2005; 12:959-69.

\section{P84}

PROTECTIVE MEASURES AND HUMAN ANTIBODY RESPONSE DURING A LARGE POULTRY OUTBREAK OF AVIAN INFLUENZA H7N3 IN BRITISH COLUMBIA, CANADA

D Skowronski, BC Centre for Disease Control; Y Li, Public Health Agency of Canada; SA Tweed, BC Centre for Disease Control; TWS Tam, Public Health Agency of Canada; M Petric, BC Centre for Disease Control; ST David, BC Centre for Disease Control; F Marra, BC Centre for Disease Control; N Bastien, Public Health Agency of Canada; SW Lee, Workplace Health and Safety Program, Health Canada; M Krajden, BC Centre for Disease Control; RC Brunham, BC Centre for Disease Control INTRODUCTION: In 2004, an outbreak of avian influenza (AI) of the H7N3 subtype occurred among poultry in British Columbia, Canada. We report compliance with recommended protective measures and associated human infections during the largest AI outbreak in Canada.

METHODS: Participation was voluntary for all persons involved in poultry depopulation. Recruitment was by advertisements at the worker deployment site, in local media and through newsletters to farmers. Sera were tested for antibody to H7N3 by microneutralization assay. A subset of 16 sera was further tested by Western blot, routine and modified hemagglutination inhibition assays, including convalescent sera from two unprotected workers with conjuncitivits from whom virus had been isolated. RESULTS: 167 persons participated between May 7 and July 26, 2004 ( $25 \%$ of workers). Of these, $12 \%$ experienced influenza-like illness and $13 \%$ experienced red/watery eyes. There was no significant association overall between illness report and exposure to infected birds. Among 65 persons entering barns with infected birds, $85 \%$ had received influenza vaccine, $74 \%$ received oseltamivir ( $48 \%$ every day while in contact with birds) and 
$85 \%, 83 \%$ and $55 \%$ reported always wearing gloves, mask or goggles. Antibody to $\mathrm{H} 7$-subtype was not detected in any sera.

CONCLUSIONS: To prevent further spread between barns and to protect workers, their families and communities from a highly-changeable virus, rigorous attention to biosecurity, occupational and public health measures is important during AI outbreaks in commercial poultry. During the $\mathrm{BC}$ outbreak, compliance with recommended protective measures, especially goggles, was incomplete. Multiple back-up precautions, including oseltamivir prophylaxis, may prevent human infections and should be readily accessible and consistently used by potentially exposed persons during AI outbreaks. Localized human AI infections may not result in serological response despite confirmed viral detection and culture. Given predictions of the spread of Eurasian-H5N1 virus into North America, lessons from the $\mathrm{BC}$ outbreak should be addressed now.

\section{P85}

\section{ESTIMATING INFLUENZA VACCINE EFFECTIVENESS USING A SENTINEL NETWORK: RESULTS FROM THE 2005-06 SEASON OF DUAL A AND B VACCINE MISMATCH IN CANADA}

D Skowronski, BC Centre for Disease Control; TL Kwindt, BC Centre for Disease Control; C Masaro, BC Centre for Disease Control; A Mak, BC Centre for Disease Control; M Petric, BC Centre for Disease Control; Y Li, Public Health Agency of Canada; R Sebastian, BC Centre for Disease Control/Public Health Agency of Canada; M Chong, BC Centre for Disease Control; T Tam, Public Health Agency of Canada; G De Serres, Quebec National Institute of Public Health

INTRODUCTION: We report a case-control design using a sentinel physician network to estimate vaccine effectiveness (VE) against laboratoryconfirmed, medically-attended influenza (LC-MAI) and provide results for the 2005-06 season of dual A and B vaccine mismatch in Canada.

METHODS: Participants were patients $\geq 5$ years of age presenting with influenza-like illness (ILI) to a sentinel physician in British Columbia, Canada between November 1, 2005 and April 30, 2006. Cases were participants in whom influenza was identified; controls tested negative for influenza A and B by PCR, R-mix and culture. Isolates were characterized by gene-sequencing and hemagglutination-inhibition (HI) assays. Odds ratios (OR) for LC-MAI in vaccinated versus non-vaccinated persons were derived with adjustment for age and chronic conditions. VE was estimated as [1-OR(vaccinated/unvaccinated)].

RESULTS: Sample included 442 patient visits: median age was 26 years, $10 \%$ were $\geq 65$ years, $15 \%$ had a chronic condition and $22 \%$ received the 2005-06 trivalent inactivated influenza vaccine $\geq 2$ weeks before ILI onset. 206 participants were positive for influenza; 107 (52\%) had influenza $\mathrm{A} / \mathrm{H} 3 \mathrm{~N} 2$ and 99 (48\%) had influenza B/Victoria lineage. Gene sequencing identified mutations away from the vaccine strain at key antigenic binding sites of the hemagglutinin (HA) protein of $\mathrm{H} 3 \mathrm{~N} 2$ isolates; the neuraminidase (NA) protein was conserved. Based on $\mathrm{HI}$ assays, three-quarters of influenza A and all B isolates were mismatched to the 2005-06 vaccine. Point estimates for VE against LC-MAI ranged between $50 \%$ and $70 \%$ for both types of influenza.

CONCLUSION: 2005-06 was the third consecutive season of vaccine mismatch based on varying $\mathrm{HA}$ for the $\mathrm{A} / \mathrm{H} 3 \mathrm{~N} 2$ component and the third also for the B component since 2001. Vaccine mismatch resulted in diminished VE but substantial cross-protection. Since it is generally more antigenically-conserved, the vaccine content and contribution of NA to overall VE should be further evaluated for both A and B components. Infrastructure for real-time epidemiologic assessment of vaccine performance, using observational study designs, is important annually and in preparation for a pandemic.

\section{P86 \\ IMPACT OF COMORBIDITIES ON RESPIRATORY-RELATED HOSPITALIZATIONS AND OFFICE VISITS DURING INFLUENZA SEASONS AMONG WOMEN OF REPRODUCTIVE AGE}

T Bodnarchuk, University of Manitoba; L Dodds; D Fell; VM Allen; A Coombs; J Scott; B Halperin; N Macdonald

BACKGROUND: Influenza is a leading cause of preventable morbidity. While comorbidities are known to increase risk of influenza complications, the magnitude of this risk in women of reproductive age has not been well studied. This study determines impact of comorbidities on rates of respiratoryrelated hospitalizations (RRH) and office visits (RRV) among these women during influenza seasons (FS) vs. non-influenza seasons (NFS).

METHODS: As part of a population-based cohort study comprising all Nova Scotia (NS) women who gave birth between 1990-2004 to examine the impact of influenza on pregnancy, we evaluated the same cohort one year prior to pregnancy. The NS Atlee Perinatal Database (NSAPD) was used to obtain comorbidities. Dates for each FS were obtained from the NS Department of Health. RRH and RRV were obtained from administrative databases linked to NSAPD. Rates of RRH and RRV were compared during FS and NFS.

RESULTS: Of 121,499 women, $10.6 \%$ had one or more comorbidity; asthma (5.5\%), anemia (3.2\%); heart disease (1\%), renal disease $(0.8 \%)$, diabetes $(0.4 \%)$. Rates of RRH did not differ between FS and NFS among healthy women (1.55 vs. 1.43/10,000 women months [WM]). Among women with comorbidities, rates of RRH were higher during FS than NFS ( 5.68 vs. $4.67 / 10,000)$. Rates of RRV were higher during FS than NFS among both healthy women ( 514.07 vs. $387.03 / 10,000 \mathrm{WM})$ and women with comorbidities ( 725.70 vs. $572.09 / 10,000 \mathrm{WM}$ ). Among 27,801 women on whom immunization data was available, only $2.8 \%$ had received flu vaccine ( $7.6 \%$ women with comorbidities vs. $2.2 \%$ healthy women).

CONCLUSION: Rates of RRH and RRV are higher in young women with COM in FS than NFS. Even in healthy women, rates of RRV are higher in FS. Despite current recommendations, only $7.6 \%$ of these women of reproductive age with comorbidities received influenza vaccine. Increased effort is needed to ensure that women with comorbidities are immunized; increased rates of RRV suggest that healthy women of reproductive age would also benefit from influenza vaccine and the costeffectiveness of universal immunization in this population should be evaluated.

\section{P87}

\section{AN EVALUATION OF THE CONCOMITANT ADMINISTRATION OF LIVE ORAL PENTAVALENT VACCINE AGAINST ROTAVIRUS GASTROENTERITIS (ROTATEQ ${ }^{\text {TM }}$ ) AND PERTACTIN-CONTAINING PERTUSSIS VACCINES} JA Mansi, Merck Frosst Canada Ltd; M Goveia, Merck Research Laboratories; M Dallas, Merck Research Laboratories; J Boslego, PATH; M Dinubile, Merck Research Laboratories; P Heaton, Merck Research Laboratories

BACKGROUND: A live human-bovine (WC3 strain) reassortant PRV expressing human serotypes $G 1, G 2, G 3, G 4$, and P[8] was evaluated in the multicenter, blinded, placebo-controlled Rotavirus Efficacy \& Safety Trial. An unexpected difference in anti-pertactin titers between PRV and placebo recipients in the prespecified analysis was observed. The following represents an off-protocol analysis of the possible interaction between PRV and acellular pertussis vaccine using a larger sample (which included the original specimens).

METHODS: Healthy infants $\sim 6$ to 12 weeks old were randomized to 3 oral doses of PRV or placebo at 4- to 10-week intervals. Subjects also received 3 doses of diphtheria and tetanus toxoids and acellular pertussis vaccine. Antibody responses to 3 pertussis antigens were measured by ELISA approximately 6 weeks postvaccination. Criteria for non-inferiority of antibody responses required the $95 \%$ confidence interval (CI) for the PRV/placebo GMT ratio had a lower bound $\geq 0.5$ (ie, no more than a 2 -fold decrease) as specified in the original protocol

RESULTS: Geometric mean antibody titres in ELISA units/mL [95\% CI] from the supplemental analysis are shown. The difference in pertactin responses 
between PRV and placebo recipients was confirmed in the original subjects, but not observed in either the additional subjects or the overall sample. CONCLUSIONS: This analysis provides evidence that PRV can be given concomitantly with pertactin-containing pertussis vaccines.

\section{P88}

\section{PREVENTING PERINATAL TRANSMISSION OF HEPATITIS B} DURING PREGNANCY IN BRITISH COLUMBIA

S Pollock, Community Medicine Resident (UBC); JA Buxton, M Gilbert, D Stark, C Eberdt, M Krajden, British Columbia Centre for Disease Control, Vancouver, BC

INTRODUCTION: Hepatitis B virus (HBV) screening is recommended in Canada for all pregnant women. HBV perinatal transmission risk is reduced by $\mathrm{HBIG}$ and vaccine prophylaxis; infants should be tested 1-6 months after final vaccine administration to determine outcome. The aims of this study were to: 1) determine the epidemiology of $\mathrm{HBV}$ in pregnant women in $\mathrm{BC}$, 2) determine prevalence of HBV perinatal transmission, and 3) evaluate the effectiveness of the $\mathrm{BC}$ perinatal program.

METHODS: Pregnant women who were hepatitis B surface antigen (HBsAg) positive between October 1, 2003 to September 30, 2004 and their infants were followed up by public health and through their physicians.

RESULTS: Prenatal tests were conducted on 45,885 women; 348 (0.76\%) were $\mathrm{HBs} A g$ positive $(13.5 \%$ were $\mathrm{HBeAg}$ reactive). Prevalence varied by region, with $4 / 16(25 \%)$ of Health Service Delivery Areas (HSDAs) accounting for 289/348 (83\%) of HBsAg positive mothers. Results were available for 97 infants; 71 (73\%) were tested for HBsAg (all negative), 92 (95\%) for antibody to $\mathrm{HBsAg}$ (anti-HBs), and 59 for antibody to hepatitis B core (anti-HBc); 52 (54\%) had all three markers tested. The anti-HBs distribtion varied: 3 infants $(3.1 \%)$ were non-responders ie, anti-HBs $<10 \mathrm{mIU} / \mathrm{L}$, and $29 / 92$ infants $(32 \%)>1000 \mathrm{mIU} / \mathrm{L}$. Anti-HBc was detected in 39 infants (66\%); 28 of 38 infants (74\%) tested before aged 12 months had anti-HBc with no HBsAg.

CONCLUSIONS: Follow-up of infants born to HBsAg positive mothers is challenging, with $>3 / 4$ of the mothers residing in 4 HSDAs. Anti-HBc may be attributed to resolved HBV infection, but as passive maternal antibodies persist for up to 18 months, anti-HBc testing should be delayed until after 18 months of age. $3 \%$ of infants did not demonstrate adequate levels of anti-HBs and reimmunization should be considered. Due to mixed vaccine delivery and follow-up systems, public health may not be aware of all infant outcomes. Standard protocols to ensure adequate vaccine coverage and comprehensive follow-up of infants should be developed through collaboration of public health and family physicians.

\section{P89}

\section{TWENTY YEAR TRENDS (1985-2004) IN THE INCIDENCE AND PREVALENCE OF ANOGENITAL WARTS IN MANITOBA: PRELIMINARY RESULTS}

E Kliewer, CancerCare Manitoba; AA Demers, CancerCare Manitoba \& University of Manitoba, Winnipeg, Manitoba; L Elliott, University of Manitoba, Winnipeg, Manitoba; M Brisson, Merck Frosst Canada \& Co, Pointe Claire Dorval, Quebec

OBJECTIVE: To describe the incidence and prevalence of anogenital warts in Manitoba for a 20 year period (1985-2004) using populationbased medical claims records.

METHODS: This study used the population registration and medical claims databases maintained by Manitoba Health. The registration file includes over $99 \%$ of the Manitoba population. The medical claims file includes all claims for payment from fee for service physicians and certain laboratories. All medical claims with a tariff code related to treatment for condylomata were extracted. Linkage within and between the two databases was performed using a unique Personal Health Identification Number. A case was considered to be incident if it was preceded by a 12-month interval free period of genital warts care. Otherwise, it was considered to be a prevalent case. An episode was considered over once a 12-month interval had lapsed without a genital wart claim. Incidence and prevalence rates were standardized to the 1991 Canadian population. Annual prevalence rates were determined as of December 31 .
RESULTS: The age-standardized incidence rate peaked in 1992 for both males $(149.0 / 100,000)$ and females $(172.3 / 100,000)$. The rates for males declined until 1999, but have since increased. The rates for females also decreased, but increased somewhat since 2002. Trends in prevalence were similar. Prevalence in 2004 was $189.7 / 100,000$ for males and 141.9/100,000 for females. The male:female incidence ratio increased from 0.76 in 1985 to 1.28 in 2004. Females aged 20-24 had the highest incidence rate in all years. Although males 20-24 also tended to have the highest rates, in some recent years this was observed among those aged 25-29.

DISCUSSION: These population-based findings provide trend information over the longest period available to date and suggest that the pattern of anogenital warts has changed over time, with the incidence becoming higher in men than women.

Study funded by Merck Frosst Canada

\section{P90}

THE EPIDEMIOLOGY OF CHILDREN HOSPITALIZED WITH INFLUENZA, 2005-6, IN IMMUNIZATION MONITORING PROGRAM, ACTIVE (IMPACT) CENTERS

$S$ Wootton, Vaccine Evaluation Center; M Mozel, University of British Columbia (UBC); K Marty, UBC; W Vaudry, Stollery Children's Hospital; D Moore, Montreal Children's Hospital; D Scheifele, UBC; S Halperin, IWK Health Centre; T Tam, Public Health Agency of Canada

BACKGROUND: Influenza is common among children and associated with significant morbidity and mortality. Hospitalization rates among healthy children, especially those $<2$ years old, approach rates of high-risk adults. The Immunization Monitoring Program, Active (IMPACT) has carried out surveillance of children hospitalized for influenza for the past three influenza seasons. This paper describes the epidemiology of children hospitalized during the 2005-6 season.

METHODS: Active surveillance for laboratory-confirmed influenza admissions among $0-16$ year old children was conducted by the IMPACT surveillance network, whose 12 centers represent over $90 \%$ of pediatric tertiary care beds in Canada. Laboratory-tests included viral culture, immunoassay, and/or molecular testing. Detailed case report forms were completed and submitted to the IMPACT data center.

RESULTS: In total, 376 children were hospitalized with influenza. The first case occurred the week of 3 December, 2005 (Ottawa) and the last 17 June, 2006 (Quebec City). Spanning 29 weeks, Canada-wide activity included one peak in mid-March. Most children were admitted in Toronto (69) followed by Edmonton (57), Quebec City (56) and Ottawa (48). In total, 235 (62.5\%) children had influenza type A and $142(37.8 \%)$ type B. Most cases were in age group 24-59 months (102, 27.1\%); 93 (24.7\%) were 6 to 23 months, 81 (21.5\%) 5-9 years, 54 (14.4\%) 0-5 months, and 46 (12.2\%) 10-16 years old. This is in contrast to the previous two seasons, when most cases occurred in children 6-23 months old. There were three deaths.

CONCLUSIONS: Influenza hospitalizations represent a significant health burden among children in Canada, especially among children age $<5$ years. The 2005-6 season occurred later than the 2004-5 season and involved more children age 24-59 months. Observations over multiple influenza seasons are required to determine if new vaccine programs for children 6-23 months will have an impact on the age profile of children hospitalized with influenza in Canada.

\section{P91}

\section{THE POTENTIAL COST-EFFECTIVENESS OF A} PROPHYLACTIC HPV-6/11/16/18 VACCINE

M Brisson, Universite Laval; N Van De Velde, Universite Laval, Quebec; MC Boily, Imperial College, London, UK; P De Wals, Universite Laval, Quebec

BACKGROUND: Clinical trials have shown prophylactic HPV vaccines to be effective against persistent infection and disease. To aid policy decisions concerning HPV vaccination, model based estimates of cost-effectiveness are needed. In the case of HPV, it is particularly important to quantify uncertainty in parameter assumptions because HPV natural history has numerous stages of disease that can vary depending on the genotype and 
screening policy. The objectives of this study were to assess the costeffectiveness of vaccination against HPV-6/11/16/18 in Canada and quantify parameter uncertainty.

METHODS: A cohort model of the natural history of HPV infection and disease was developed. An extensive fitting procedure was performed, which identified 155 different parameter sets that fit simultaneously Canadian HPV type-specific data for infection, CIN, cervical cancer and genital warts $(\mathrm{GW})$. Quality-Adjusted Life-Years (QALYs) lost were calculated using data available in the literature. Unit costs were applied to the predicted HPV-associated health care resource use. The cost-effectiveness of vaccination was estimated for various age cohorts and probabilistic sensitivity analysis was performed (155,000 simulations).

RESULTS: Vaccinating 12-year-old girls (vaccine efficacy $=100 \%$, no waning) against HPV-6/11/16/18 is estimated to cost $\$ 6,268$ (80\%CrI:4,626-7,651) to $\$ 17,435$ (80\%CrI:7,651-33,481) per QALY gained, when varying the cost per course between $\$ 300$ and $\$ 600$. Using $\$ 50,000$ per QALY gained as strong evidence for cost-effectiveness, HPV$6 / 11 / 16 / 18$ vaccination was estimated to be cost-effective under a wide range of parameter assumptions and vaccination scenarios. Results were most sensitive to the duration of vaccine protection and the QALY-lost per case of GW.

CONCLUSION: This is the first cost-effectiveness study that incorporates probabilistic sensitivity analysis to assess the robustness of model predictions. Results suggest that HPV-6/11/16/18 vaccination is cost-effective.

\section{P92 \\ ESTIMATING THE NUMBER NEEDED TO VACCINATE TO PREVENT HPV RELATED DISEASE AND MORTALITY}

M Brisson, Universite Laval; N Van De Velde, Universite Laval, Quebec; MC Boily, Imperial College, London, UK; P De Wals, Universite Laval, Quebec

BACKGROUND: The number needed to treat (NNT) has been used to help describe the population outcome of chronic disease treatments. A similar measure, number needed to vaccinate (NNV), can be applied to vaccine preventable diseases. The aim of this study was to estimate the NNV to prevent HPV related diseases and mortality in Canada.

METHODS: A cohort model of the natural history of HPV infection and disease was developed. Model simulations were based on 155 different parameter sets that fit Canadian HPV type-specific data for infection, CIN, cervical cancer and genital warts. NNV was calculated as the number of women (within a specific age cohort) that are needed to be vaccinated to prevent an HPV related event during their lifetime.

RESULTS: For 12-year-old girls, the NNV (vaccine types=HPV$6 / 11 / 16 / 18$, vaccine efficacy $=100 \%$, no waning) to prevent a genital wart episode, diagnosed CIN1, diagnosed CIN2/3, cervical cancer case, cervical cancer death and life-year lost is estimated to be 4 (80\%CrI:3-8), 19 (80\%CrI:9-45), 58 (80\%CrI:30-113), 241 (80\%CrI:154-456), 528 $(80 \% \mathrm{CrI}: 317-1,071)$ and $23(80 \% \mathrm{CrI}: 15-41)$ respectively. If an HPV$16 / 18$ vaccine is used (vaccine efficacy $=100 \%$, no waning), then the NNV to prevent a diagnosed CIN1 increases to 25 and genital warts are not prevented. Results were most sensitive to the duration of vaccine protection and the age at vaccination.

CONCLUSION: NNV can be a very helpful tool for clinicians and decision makers to illustrate the potential benefit of HPV vaccination as they are a composite measure of the age-specific background rates of disease and vaccine efficacy. However, the NNV results presented here should only be generalizable to countries and regions with similar HPV epidemiology to Canada. Finally, the results presented here were based on a cohort model and therefore results may underestimate the true NNV since herd-immunity effects are not included.

\section{P93 \\ MEASURING HERPES ZOSTER (HZ) AND POST-HERPETIC NEURALGIA (PHN) ASSOCIATED BURDEN OF ILLNESS, HEALTH CARE UTILIZATION AND COSTS IN CANADA}

M Brisson, Universite Laval; RV Johnson, United Bristol Hospitals, Bristol, United Kingdom; MJ Levin, University of Colorado, Denver, USA; MN Oxman, University of California, San Diego, California, USA; KE Schmader, Duke University, Durham, North Carolina, USA; DM Patrick, University of British Columbia, Vancouver, British Columbia; C Koch, M Senecal, JA Mansi, Merck Frosst Canada Ltd, Kirkland, Quebec

AIM: Describe MASTER, a prospective study that measures HZ-associated burden of illness, health care utilization and costs, and present baseline results.

METHODS:

- Study type: Multi-center prospective cohort study.

- Recruitment dates: October 2005 to June 2006.

- Inclusion criteria: Patients $\geq 50$ years of age presenting with either $\mathrm{HZ}$ rash, zoster associated pain or PHN (pain $>90$ days).

- Recruitment sites: 90 physician offices across Canada.

- Questionnaires and follow-up: 10 self-administrated questionnaires over 6 months.

- Study data: Severity and duration of pain (using Zoster Brief Pain Inventory and Initial Zoster Impact questionnaires); QALYs (qualityadjusted life-years lost using the EQ-5D); health care utilization; work loss.

RESULTS: As of May 18, 453 patients have been recruited (59\% female, mean age 68 years). Of these, $69 \%$ presented at baseline with acute HZ and $31 \%$ with PHN. For patients with acute HZ (PHN), mean average, worst and least pain in the last 24 hours (worst imaginable pain=10) was 4.0 (3.6), 5.4 (4.7) and 2.6(2.2), respectively. Mean HZ (PHN) pain interference with general activity, mood, sleep, and enjoyment of life (0-to-10 point scale) was $3.7(2.8), 3.6(2.7), 4.3(3.4)$ and $4.2(3.5)$, respectively. The mean QALY-weight lost was -0.18 and -0.16 for patients with acute $\mathrm{HZ}$ and PHN, respectively.

CONCLUSION: HZ and PHN cause substantial acute and chronic pain and significantly affect quality of life. The results form the baseline for a 6-month prospective study.

\section{P94 \\ MEASURING THE PAIN AND SEVERITY ASSOCIATED WITH THE PRODROMAL PHASE OF HERPES ZOSTER (HZ) IN CANADA: A PROSPECTIVE COMMUNITY BASED STUDY}

M Brisson, Universite Laval; RW Johnson, United Bristol Hospitals, Bristol, United Kingdom; MJ Levin, University of Colorado, Denver, USA; MN Oxman, University of California, San Diego, California, USA; KE Schmader, Duke University, Durham, North Carolina, USA; DM Patrick, University of British Columbia, Vancouver, British Columbia, Canada; M Senecal, C Koch, JA Mansi, Merck Frosst Canada Ltd, Kirkland, Quebec

AIM: Describe the frequency, severity and duration of prodromal pain in $\mathrm{HZ}$ and identify associated risk factors.

METHODS: From October 2005 to June 2006, 284 patients ( $\geq 50$ years of age) presenting with a $\mathrm{HZ}$ rash were recruited at 90 clinics across Canada. Morbidity associated with the prodromal phase was measured using the Initial Zoster Impact Questionnaire (IZIQ).

RESULTS: 274 patients answered the IZIQ, $60 \%$ of whom were female. The mean age of patients was 66 years $(\mathrm{SD}=11)$. Prodromal pain was reported in $68 \%$ of cases. Of these, $39 \%$ reported having more than 18 hours of pain per day. On average, prodromal pain appeared 4.4 days $(\mathrm{SD}=3.6)$ before rash onset. Average and worst pain during the prodrome were $5(\mathrm{SD}=2.8)$ and $6(\mathrm{SD}=2.9)$ out of 10 respectively (10 is worst imaginable pain). Age and gender did not significantly affect the occurrence of prodromal pain. Patients with prodromal pain were more likely to experience pain $(98 \%$ vs. $83 \%, \mathrm{p}$-value $<0.001)$ and suffered greater average pain $(5.5$ vs. 4.7 , $\mathrm{p}$ value $<0.05$ ) after the onset of $\mathrm{HZ}$ rash than those who did not report prodromal pain. 
CONCLUSION: This study suggests that the pain during the prodromal phase of $\mathrm{HZ}$ is significant, and that the presence of prodromal pain is a risk factor for greater pain after the onset of $\mathrm{HZ}$ rash. The patients in this study will be followed up for 6 months to determine whether the presence of prodromal pain is a risk factor for PHN and duration and severity of $\mathrm{HZ}$ associated pain.

\section{P95}

\section{CHARACTERIZATION OF REGULATORY T CELLS FOLLOWING INFLUENZA VACCINATION}

C Card, University of Manitoba; Y Keynan, University of Manitoba; SA Koesters, University of Manitoba, Winnipeg, Manitoba; FA Plummer, Public Health Agency of Canada; University of Manitoba; KR Fowke, University of Manitoba, Winnipeg, Manitoba INTRODUCTION: Regulatory T cells (Tregs) are a subset of circulating CD4+ $\mathrm{T}$ cells that inhibit immunopathology and autoimmune disease. Recently, Tregs have been implicated as mediators of immune responses to pathogens, and have been shown to exert a suppressive effect on proliferative and cytokine responses of CD4+ and CD8+ T cells. In this study, Treg populations are characterized by multicolour flow cytometry in influenzavaccinated individuals. The objectives of the study are to determine the frequency and phenotype of Tregs in each of the study subjects and to determine if there is a correlation between the magnitude of responses to influenza antigens and the frequency of Tregs in peripheral blood.

METHODS: Study subjects are healthy influenza-vaccinated volunteers from the University of Manitoba. Peripheral blood mononuclear cells (PBMC) are isolated from whole blood and stimulated with SEB, whole influenza virus and peptides of immunodominant influenza epitopes. Ten colour flow cytometry is used to determine the frequency and phenotype of Tregs in the PBMC population before and after antigen stimulation. Antigen-specific responses are characterized using 10-colour flow cytometric detection of cytokine production, activation and memory markers. RESULTS: Ten colour flow cytometry has been established in the lab and is currently being applied to samples. Results of Treg phenotyping and influenza-specific immune responses will be presented.

CONCLUSIONS: The role of regulatory $\mathrm{T}$ cells in mediating immune responses to pathogens such as influenza is currently under investigation. Determining the effects of these cells on memory responses to virus challenge following vaccination could provide valuable information for future vaccine design and treatment options.

\section{P96}

EFFECTIVENESS OF INFLUENZA VACCINE IN PREVENTING HOSPITALIZATION OF CHILDREN 6-23 MONTHS OF AGE

G De Serres, Institut National de santé publique du Québec; S Halperin, IWK Grace Hospital; D Skowronski, BC Center for Disease Control; P Déry, CHUQ-CHUL; W Vaudry, Stollery Children's Hospital; D Moore, Montreal Children's Hospital; A McGeer, Mount Sinai Hospital; B Duval, Institut National de santé publique du Québec; J Bettinger, BC Children's Hospital; T Tam, Public Health Agency of Canada; D Scheifele, BC Children's Hospital INTRODUCTION: Infants and toddlers aged 6 to 23 months are reported to suffer high rates of hospitalization due to influenza. Beginning in 2004, routine influenza vaccination for infants/toddlers was therefore recommended in Canada. No data exist to support efficacy of the influenza vaccine in preventing hospitalization in this age group. The objective of this pilot study was to estimate vaccine effectiveness (VE) against hospitalization due to influenza among infants and toddlers.

METHODS: This prospective case-control pilot was conducted in 8 hospitals belonging to the IMPACT network and 2 community hospitals from the Toronto area. Participants were children $<24$ months of age on November 1, 2005 (beginning of the vaccination campaign) and $\geq 6$ months of age at the end of January, 2006 (end of the typical vaccination period). Children vaccinated after January 1 2006, non-Canadian residents or those from outside the geographical location of the center, children for whom information could not be obtained because of language barrier and nosocomial influenza cases were excluded. Cases were children hospitalized for laboratory-confirmed influenza illness or complication. Controls were children hospitalized for acute respiratory infection (ARI) not caused by influenza as evidenced by negative laboratory results for influenza. RESULTS: 41 influenza cases (28 influenza A and 13 B) and 78 controls agreed to participate. Compared to controls, cases were more often male, born full-term, lived with $\geq 3$ adults, lived with $\geq 3$ other children and less often attended daycare. The crude overall VE in children who received $\geq 1$ dose of the 2005-2006 influenza vaccine was $-0.2 \%$ [-142\%; 59\%] but decreased to $-27 \%$ [-289\%; $59 \%$ when restricted to children who received two doses. VE did not vary substantially by influenza subtype (A or B). VE varied between $9 \%$ and $20 \%$ after adjustment for underlying medical conditions and other confounders but never approached statistical significance. VE in children aged $\geq 18$ months at the time of hospitalization was not greater than in those 6-11 months or 12-17 months.

CONCLUSION: The observed trend in VE was not indicative of great benefit, if any, to infants/toddlers in preventing influenza-related hospitalization. Mismatch between vaccine and circulating strains, both A and B, in 2005-06 may have contributed to poor VE estimates.

\section{P97}

\section{ADDRESSING A PUBLIC HEALTH PRIORITY: FIRST CANADIAN RESEARCH AGENDA ON INFLUENZA PREVENTION, CONTROL AND MANAGEMENT}

D Scheifele, Vaccine Evaluation Center; T Tam, Public Health Agency of Canada, Ottawa, Ontario; B Singh, Canadian Institutes of Health Research, Ottawa, Ontario; G Bjornson, Canadian Association for Immunization Research and Evaluation; L Paddle, Public Health Agency of Canada, Ottawa, Ontario; C Richardson, Canadian Institutes of Health Research, Ottawa, Ontario

BACKGROUND: Canadian and international vaccine experts and researchers from many disciplines gathered in a two day workshop in 2005 to determine the national research priorities that will inform pandemic and inter-pandemic influenza prevention and control strategies.

METHODS: Over 70 influenza experts, policy-makers, clinicians, and researchers attended the workshop. Initial plenary sessions provided overviews of current influenza knowledge and research activities, from basic virology to disease control with vaccines, antivirals and non-pharmaceutical interventions. Participants were assigned to multidisciplinary groups to identify key knowledge gaps and research needs in major topic areas. Groups were asked to identify and reach consensus on 3-5 research questions per topic which were then debated in plenary to identify ten national research priority areas and the required infrastructure.

RESULTS: The discussion groups identified 20 unique research questions and 16 areas of infrastructure/capacity gaps. Ultimately, the ten research priority areas identified were: studies of influenza virus genetics and ecology, virus shedding and transmission, disease avoidance measures, improved rapid diagnosis and case management, health care system surge capacity, development and optimal use of antivirals, improved annual vaccines, comparison of vaccination program strategies and studies of domestic pandemic influenza vaccines. Key infrastructure needs identified included networks for evaluation of vaccines, antiviral drugs and infection control measures.

CONCLUSIONS: The workshop achieved consensus on ten research priority areas for Canadian researchers and funding agencies, and identified where Canada may provide valuable contributions to international research activities and where it must address unique domestic issues. 


\section{P98}

\section{SEROTYPE DISTRIBUTION OF ROTAVIRUS ASSOCIATED GASTROENTERITIS IN CANADA: A COMMUNITY BASED STUDY}

M Sénécal, Merck Frosst Canada Ltée.; MH Lebel, Centr hospitalier mère-enfant Sainte-Justine, Montréal, Québec; J Yaremko, McGill University, Montreal, Québec; R Wong, University of Toronto, Toronto, Ontario; LA Gallant, University of Toronto, Toronto, Ontario; H Garfield, University of Toronto, Toronto, Ontario; DJ Ableman, University of British Columbia, Vancouver, British Columbia; RL Ward, Cincinnati Children's Hospital Medical Center, Cincinnati, Ohio, USA; J Sampalis, JSS Research, Montreal, Quebec; M Brisson, Université Laval, Québec, Québec; JA Mansi, Merck Frosst Canada Ltd, Montréal, Québec

BACKGROUND: Rotavirus is the leading cause of dehydrating gastroenteritis (GE) in children. Recently, a large placebo-controlled trial has shown the safety and the efficacy of a pentavalent rotavirus vaccine reassortants of human serotypes G1, G2, G3, G4 and P1. To better estimate the potential impact of this vaccine at a population level, describing the country-specific distribution of rotavirus serotypes in community practice is necessary. The aim is to describe the distribution of rotavirus serotype infections in children under 3 consulting a physician for RGE in Canada. METHODS: Between January and June 2005, 421 children aged 3 years and under presenting with GE were prospectively recruited through 60 clinics across Canada. 395 stool specimens were tested for rotavirus antigen using an enzyme immunoassay (EIA). Samples found to be rotavirus positive were tested by reverse transcription-polymerase chain reaction (RT-PCR) of a portion of the VP7 gene segment and product sequencing, with G-type determination based on sequence homology.

RESULTS: Of the 395 rotavirus antigen testing, 369 were conclusive: 200 samples $(54.2 \%)$ were rotavirus positive and 169 (45.8\%) were rotavirus negative. The $\mathrm{G}$-serotype distribution obtained in the Canadian community practice between January 2005 and June 2005 is as follow: 55.4\% G1, 3.4\% G2, 10.3\% G3, 22.3\% G4, 8\% G9 and 0.6\% G12.

CONCLUSION: Our study demonstrates that several different rotavirus G-serotypes were prevalent in Canada during the 2005 rotavirus season. As expected, the vast majority RGE (88.0\%) were of G-serotype 1, 3 and 4 . However, the prevalence of G-serotype 4 was higher than that reported in other developed countries. The data underscores the important contribution of non-G1 serotypes such as G3, G4 and G9 to the burden of rotavirus disease during a given rotavirus.

\section{P99}

\section{BURDEN OF ROTAVIRUS ASSOCIATED GASTROENTERITIS IN CANADIAN FAMILIES: A PROSPECTIVE COMMUNITY BASED STUDY}

M Sénécal, Merck Frosst Canada Ltée.; M Brisson, Merck Frosst Canada Ltd, Montreal, Quebec; Université Laval, Québec, Québec; MH Lebel, Centre hospitalier mère-enfant Sainte-Justine, Montréal, Québec; J Yaremko, McGill University, Montreal, Quebec; R Wong, University of Toronto, Toronto, Ontario; LA Gallant, University of Toronto, Toronto, Ontario; H Garfield, University of Toronto, Toronto, Ontario; DJ Ableman, University of British Columbia, Vancouver, British Columbia; RL Ward, Cincinnati Children's Hospital Medical Center, Cincinnati, Ohio, USA; J Sampalis, JSS Research, Montreal, Quebec; JA Mansi, Merck Frosst Canada Ltd, Montréal, Québec

BACKGROUND: Recently a large clinical trial has shown the efficacy and safety of vaccination against rotavirus gastroenteritis (RGE). The economic and psychosocial impact of RGE on Canadian families has not been evaluated, but such data are essential to perform economic evaluation of vaccination. The aim is to measure the burden of RGE in Canadian families. METHODS: From January to June 2005, 421 children under 3 years of age presenting with GE symptoms were recruited through 60 clinics across Canada. Rotavirus antigen testing was completed on 395 stool samples. QoL, work loss and household contacts was evaluated via questionnaires filled by caregivers at baseline, week one and two. A Visual Analogue
Scale (VAS) and the Health Utilities Index mark 2 (HUI2) was used to evaluate the quality of life (QoL) lost in children with RGE. Caregiver QoL was measured using the EuroQoL and a VAS. QoL lost was assessed by taking the difference between measurements at baseline and at week two. RESULTS: For 111 out of 200 children (56\%) that were rotavirus-positive, at least one parent had to miss work; these families lost 2.9 work days on average. $46.5 \%$ of families reported other cases of GE in the household within \pm 2 weeks of the RGE index case; 1.7 additional GE cases occurred in these household, on average. Depending on the measurement tool, RGE reduced the QoL of children by $8 \%$ (HUI2) and 31\% (VAS) and QoL of Caregiver's by $10 \%$ (EuroQoL) and $12 \%$ (VAS).

CONCLUSION: Our results show that, in Canada, RGE has a significant impact on the QoL of not only the child but also on the parents. Furthermore, RGE is associated with substantial work lost and may be frequently transmitted to household members.

\section{P100}

\section{CYTOMETRIC BEAD ARRAY ANALYSIS FOR THE DETECTION OF SERUM ANTI-INFLUENZA IMMUNOGLOBULIN-A1}

T Bodnarchuk, University of Manitoba; Y Keynan, University of Manitoba; S Wayne, University of Manitoba; Y Lee, Public Health Agency Canada NML; FA Plummer, Public Health Agency Canada NML; KR Fowke, University of Manitoba

OBJECTIVE: Mucosal and serum production of neutralizing IgA antibodies is a natural immune response to influenza infection. Anti-flu IgA prohibits viral entry and subsequent infection. Current inactivated trivalent vaccines given intra-muscularly are relatively poor stimulants for $\operatorname{IgA}$ production. Immunity to future viral challenge is dependant on the presence of type specific anti-flu neutralizing antibodies (specifically IgA). Quantization and determination of antigen specificity of systemic and mucosal immune response to influenza vaccination is beneficial for future vaccine development. Previous methods to acquire this information proved costly, time-consuming and were sample exhaustive. The benefits of cytometric bead array (CBA) analysis are numerous. The multiplex capabilities of the system conserve time, money and sample while generating statistically powerful data.

METHODS: Latex microspheres contain a ratio of two dyes, with up to 100 possible combinations, giving each set a unique spectral address. Bead sets are coupled to known flu antigens (HA). Individual bead sets are mixed together to simultaneously assay serum samples for the presence of antibodies. The beads are incubated in serum samples to allow binding of host flu-specific antibodies. The beads are incubated with biotinylated mouse anti-human IgA monoclonal antibodies (mAH-IgA), binding host IgA flu specific antibody. Streptavidin-PE is washed over the beads to label mAH-IgA. Using flow cytometry, one laser detects the bead and its spectral address. A second laser detects the presence of the reporter.

RESULTS: Preliminary results document the ability of CBA to detect and quantize the $\operatorname{IgA}$ immune response to vaccine strains of influenza. A/NewCaledonia/20/99; A/Wyoming/2/2003; B/Hong Kong/330/2001 as well as strains not frequently encountered and absent from vaccine preparations. CONCLUSIONS: CBA technology allows the quantization and determination of antigen specificity of $\operatorname{IgA}$ immune response to influenza vaccination. Cross-reacting antibody response is detected and characterization of that response may prove useful for vaccine development.

\section{P101}

\section{ADVERSE EVENTS FOLLOWING HEPATITIS B} IMMUNIZATION OF CHILDREN AGED 7 TO < 13 YEARS IN CANADA (JAN 1997 - DEC 2005)

J Nkanza, Public Health Agency of Canada; BJ Law, Public Health Agency of Canada

INTRODUCTION: Canadian school-based universal Hepatitis B (HB) immunization programs were initiated in British Columbia in 1992 and country-wide by 1998 . This report summarizes the national adverse event reporting profile for $\mathrm{HB}$ vaccine when administered alone to children aged 7 to $<13$ years. 
METHODS: All reports of Adverse Events Following Immunization (AEFI) submitted to the Public Health Agency of Canada involving HB vaccine given from January 1, 1997 to December 31, 2005 were reviewed and the subset involving $\mathrm{HB}$ alone given to children aged 7 to $<13$ years of age analyzed using SAS software.

RESULTS: Of 4416 AEFI reports following HB vaccine, 1969 (44.6\%) involved children aged 7 to $<13$ years with $\mathrm{HB}$ the only vaccine given for 1826 (93\% of 1969). The most frequently reported AEFIs were allergic reactions $28.6 \%$ (523), injection site reactions $22.4 \%$ (409), fever $22.1 \%$ (404), rash $20.5 \%$ (374), severe vomiting and/or diarrhea $12.0 \%$ (220), urticaria $12.0 \%(220)$, headache $11.5 \%(211)$, dizziness $6.9 \%(127)$ and nausea $6.1 \%(111)$. There were $7(0.4 \%)$ reports of anaphylaxis. Outcome at the time of reporting was specified for 1345 (74\%), including 1,229 completely recovered, 87 recovering, one fatality and 45 residual effects. Medical attention was specified for 1349 (74\%), including 37 hospitalized, 33 emergency room visits, 681 non-urgent outpatient visits, one help line call and 597 requiring no medical care. Of 16 reports with sufficient detail for causality assessment, the association with HB vaccine was judged to be unclassifiable for 2, very likely for one, possible for 2, unlikely for 5 and unrelated for 6, including the single fatal case due to myocarditis syndrome. CONCLUSIONS: Most nationally reported AEFIs were either local reactions or less serious systemic reactions. These data support the safety of $\mathrm{HB}$ vaccine as given to school-age children in Canada.

\section{P102}

\section{COMPARISON OF 0-1 MO AND 0-2 MO VACCINATION SCHEDULES WITH HEPATITIS B VIRUS SURFACE ANTIGEN+1018 IMMUNOSTIMULATORY PHOSPHOROTHIOATE OLIGONUCLEOTIDE}

Scott Halperin, Dalhousie University; JM Langley, Dalhousie University; S McNeil, Dalhousie University; R Sani, Dalhousie University; B Smith, Dalhousie University; D Bomba, Dynavax Technologies Corporation; E Martins, Dynavax Technologies Corporation

BACKGROUND: Two doses of Hepatitis B Virus Surface Antigen plus 1018 Immunostimulatory Phosphorothioate Oligonucleotide (HBV-ISS; Heplisav) administered at $0-2$ months has been shown to provide seroprotection earlier than 3 doses of a licensed hepatitis B vaccine. A 0-1 month schedule of HBV-ISS could provide several benefits including ease of administration and earlier protection.

METHODS: Healthy, seronegative $18-40$ year old adults were randomly assigned to receive HBV-ISS on a $0-1$ or $0-2$ month schedule; placebo was administered at 2 and 1 months respectively for blinding purposes. Local and systemic reactogenicity events and adverse events were collected following each injection. Antibodies were measured at baseline, 1, 2, 3, and 8 months.

RESULTS: 41 participants were enrolled; 19 were randomized to the 0-1 and 22 to the $0-2$ groups. There were $58 \%$ males and $59 \%$ females; mean age of 27.2 and 30.5 years, respectively. Rates of injection site reactions (redness, swelling, pain) and general reactions (fever, malaise, headache, fatigue) were similar after each dose of HBV-ISS, regardless of the schedule, but tended to be more frequent than after placebo. A total of $22.2 \%$ of $0-1$ and $27.3 \%$ of $0-2$ recipients had protective $(\geq 10 \mathrm{mIU} / \mathrm{mL})$ antibody levels 1 month post dose 1 (GMCs of 2.34 and $3.07 \mathrm{mIU} / \mathrm{mL}$ respectively). By 1 month post dose 2, $94.4 \%$ of $0-1$ and $100 \%$ of $0-2$ recipients had protective antibody levels (GMCs of 222 and $3276 \mathrm{mIU} / \mathrm{mL}$, respectively). At month $3,100 \%$ of $0-1$ recipients had protective antibody levels (GMC of $418 \mathrm{mIU} / \mathrm{mL}$ ). At month 8, all participants persisted with protective antibody levels; differences in GMCs were not apparent $(0-1$ group $=458 \mathrm{mIU} / \mathrm{mL} ; 0-2$ group $=861 \mathrm{mIU} / \mathrm{mL})$. CONCLUSIONS: Equivalent rates of early and sustained seroprotection are achieved more conveniently using a 0-1 than a 0-2 month vaccination schedule with HBV-ISS. The significant difference in GMCs observed 1 month post dose 2 does not persist at month 8 .

\section{P103 \\ DEVELOPMENT OF A PROTOLLIN ${ }^{\mathrm{TM}}$-BASED INACTIVATED MUMPS VIRUS VACCINE FOR NASAL ADMINISTRATION: MOUSE SAFETY AND IMMUNOGENICITY}

K Young, McGill University Health Centre Research Institute, Montreal, Quebec; S Nzula, McGill University Health Centre Research Institute, Montreal, Quebec; C Rodeheffer, McGill University Health Centre Research Institute, Montreal, Quebec; A Brewer, McGill University Health Centre Research Institute, Montreal, Quebec; D Burt, ID Biomedical of Canada (GSK), Laval, Quebec; BJ Ward, McGill University Health Centre Research Institute, Montreal, Quebec

INTRODUCTION: The incidence of mumps declined with the inclusion of mumps vaccine in trivalent MMR in the late 1960s. Mumpscontaining vaccines have significant limitations, however: 1) caution must be exercised in immunocompromised hosts; 2) maternal antibodies inhibit seroconversion; 3) serious side effects can occur (aseptic meningitis and post-infectious encephalomyelitis); and 4) the vaccine is administered by needle. Recent outbreaks of mumps in North America highlight this vaccine's limitations. An inactivated nasal vaccine would deal with many of these problems. Proteosome-based adjuvants are well suited for this purpose. METHODS: Eight-week old BALB/c female mice were vaccinated intranasally (IN) or intramuscularly (IM) with three doses of split Jones mumps virus $(\mathrm{MuV})$ antigen $(2,4$, or $8 \mu \mathrm{g})$ combined with Protollin ${ }^{\mathrm{TM}}$ at a 1:1 ratio. Mice were monitored to assess vaccine safety, and sera and mucosal secretions were obtained at intervals to assess $\mathrm{MuV}$-specific immunity.

RESULTS: Mice exhibited no behavioural changes or weight loss throughout the study. IN and IM administration of Protollin ${ }^{\mathrm{TM}}-\mathrm{MuV}$ vaccine induced serum antibodies, in an antigen-concentration-dependent manner, capable of neutralizing $\mathrm{MuV}$ in vitro. Doses of 2 (IN), 4 (IN and IM), and 8 (IN) $\mu$ g Protollin ${ }^{\mathrm{TM}}-\mathrm{MuV}$ elicited serum neutralizing antibody titers of 26, 69, 83 and 539 units, respectively. Surprisingly, no serum neutralizing antibody response was elicited in mucosal secretions, at any dose. Evaluation of serum IgG and IgG isoforms, mucosal IgA, cytokine profiles, and antigen-specific splenocyte lymphoproliferation is currently underway. CONCLUSIONS: The intranasal Protollin ${ }^{\mathrm{TM}}-\mathrm{MuV}$ vaccine was safe and immunogenic. The vaccine elicited a serum neutralizing antibody response administered IN or IM. Studies are underway to combine our MuV antigen with measles and rubella antigens to create an inactivated nasal MMR vaccine. Such a vaccine could help to resolve many of the limitations of the current MMR.

\section{P104}

THE BURDEN OF VARICELLA AND ZOSTER IN BRITISH COLUMBIA 1994-2003: BASELINE ASSESSMENT PRIOR TO UNIVERSAL VACCINATION

B Edgar, BC Centre for Disease Control (BCCDC); E Galanis, BCCDC; C Kay, BCCDC; D Skowronski, BCCDC; M Naus, BCCDC; D Patrick, BCCDC

BACKGROUND: Varicella vaccine became available in Canada in January 1999. Until 2004, the majority of varicella vaccinations were paid for privately in BC. In September 2004, varicella vaccine was provided free of charge to high-risk individuals and in January 2005, a publicly funded universal program was launched. There is some concern that universal vaccination could lead to an increase in the average age of infection where risk of complications is greater and a temporary increase in the incidence of zoster.

METHODS: Administrative data for physician visits, hospitalizations and deaths from 1994 to 2003 was examined to assess trends and the burden of illness associated with varicella and zoster disease in $\mathrm{BC}$, prior to the introduction of universal vaccination in 2005.

RESULTS: Incidence of physician visits for varicella (32.5 visits per 10,000 population/year, average 1994-2003) decreased over the study period while visits for zoster (28.9 visits per 10,000 population/year, average 1994-2003) increased. Hospitalization rates for both varicella ( 0.4 hospitalizations per 10,000 population/year, average 1994-2003) and zoster (1.0 hospitalizations 
per 10,000 population/year, average 1994-2003) were decreasing, but a more dramatic decline in rates is expected with the introduction of universal vaccination in 2005. Approximately one-third (34.5\%) of varicella hospitalizations and nearly half (43.7\%) of all zoster hospitalizations had associated complications. During the 10-year period, there were 7 deaths associated with varicella (highest age-specific mortality rate in children $1-4$ years of age $0.54 /$ million, average $1994-2003$ ). Zoster was the underlying cause of death in $29 \mathrm{BC}$ residents, 28 of whom were 65 years of age or older (mortality rate of 5.5/million, average 1994-2003).

CONCLUSION: In the absence of incidence data, administrative data sources are useful for monitoring the incidence of varicella and zoster. These baseline data will be used to examine the changing epidemiology of the infection as well as ensure high vaccine coverage and effectiveness.

\section{P105 \\ PHENOTYPIC CHARACTERIZATION OF MEMORY CD8+ T-CELL RESPONSES TO INFLUENZA VIRUS IMMUNIZATION}

Y Keynan, University of Manitoba; CM Card, Department of Medical Microbiology University of Manitoba; Y Li, Public Health Agency of Canada (PHAC); FA Plummer, PHAC; KR Fowke, Department of Medical Microbiology, University of Manitoba Influenza-specific CD8+ T-cells assist in the elimination of virus and subsequent host recovery. In the absence of these cells viral clearance is delayed. The response is directed primarily at six major epitopes, two of which comprise the prominent CD8+ sets, namely NP 366-374 and PA 224-233.

Memory CD8+ T-cells are comprised of heterogenous populations differentiated by lymph node homing properties, location, function and cytokine receptor expression profiles. Central memory $(\mathrm{T} \mathrm{cm})$ are CD45RO, CD62Lhi, CCR7hi and circulate between lymphoid organs while the peripherally distributed effector memory (Tem) are CD45RO, CD62Llo, CCR7lo. These subsets differ in their function, with Tem expressing perforin and cytotoxicity while $\mathrm{Tcm}$ acquire effector function after in-vitro stimulation. Both subsets produce IFN $\gamma$ and TNF $\alpha$ but only Tcm secrete IL-2 $(1,2)$.

The contribution of the Tem to recall response is greater in the early memory phase while the $\mathrm{T} \mathrm{cm}$ dominate the later memory time points.

METHODS: Healthy subjects were recruited in Winnipeg after institution ethics board approval. PBMC were stimulated with whole influenza viruses. H1N1-A/New Caledonia/20/99; H3N2-A/Wyoming/2/2003 and with two epitopes derived from influenza nucleoprotein-NP 366-374 and acid polymerase-PA 224-233.

The data was acquired using LSR2 and analyzed on FACSDiva software. Gating on CD8+ lymphocytes the IFN $\gamma$ and IL-2 response as well as the memory subset of the responding cells are determined.

RESULTS: Preliminary results document the memory phenotype following influenza vaccination. Virus specific memory CD8+ T-cells differ in the cytokine response induced by peptide stimulation. CD62L expression profile among memory CD8+ T-cells varies for the different immunodominant epitopes.

CONCLUSIONS: The use of multiparametric polyfunctional flowcytometry allows better definition of the effector response as well as characterization of the memory phenotype of responding cells. Information gained regarding the memory response may contribute to better vaccine design.

References:

1. Hikono $\mathrm{H}$ et al. T-cell memory and recall responses to respiratory virus infections. Immunol Rev 2006;211:119-32.

2. Kedzierska K et al. Establishment and recall of CD8+ T-cell memory in a model of localized transient infection. Immunol Rev 2006;211:133-45.
P106

SEVERITY OF ROTAVIRUS ASSOCIATED GASTROENTERITIS AND RELATED HEALTH CARE RESOURCE UTILIZATION IN CANADA: A PROSPECTIVE STUDY IN COMMUNITY PRACTICE

M Sénécal, Merck Frosst Canada Ltée.; MH Lebel, Centre hospitalier mère-enfant Sainte-Justine, Montréal, Québec; J Yaremko, McGill University, Montreal, Quebec; R Wong, University of Toronto, Toronto, Ontario; LA Gallant, University of Toronto, Toronto, Ontario; H Garfield, University of Toronto, Toronto, Ontario; DJ Ableman, University of British Columbia, Vancouver, British Columbia; RL Ward, Cincinnati Children's Hospital Medical Center, Cincinnati, Ohio, USA; J Sampalis, JSS Research, Montreal, Quebec; M Brisson, Université Laval, Québec, Québec; JA Mansi, Merck Frosst Canada Ltd, Montréal, Québec

OBJECTIVE: Rotavirus is the leading cause of severe gastroenteritis (GE) in children. To evaluate the potential impact of vaccination at a population level, a thorough understanding of the burden of disease is required. This study describes the severity of RGE and related Health Care Resource Utilization (HCRU) in Canadian community practices.

METHODS: Between January and June 2005, 421 children under 3 years of age presenting with GE ( $\geq 3$ watery or looser-than-normal stools and/or forceful vomiting within a 24-hour period) were prospectively recruited through 60 clinics across Canada. Caregivers were asked to collect and return stool samples. 395 stool specimens were tested for rotavirus antigen using an enzyme immunoassay. Positive specimens were typed by reverse transcription-polymerase chain reaction. GE symptoms and HCRU were reported by caregivers at baseline and for 14 days of follow-up using daily diary cards.

RESULTS: Of 369 conclusive test results, 200 (54.2\%) were rotavirus positive $(\mathrm{R}+)$. Compared to rotavirus negative $(\mathrm{R}-), \mathrm{R}+$ were more likely to have diarrhoea $(100 \%$ vs. $95.9 \%, p=0.0037)$, vomiting $(85.5 \%$ vs. $50.9 \%, \mathrm{p}<0.001)$, fever $(62.0 \%$ vs. $34.3 \%, \mathrm{p}<0.001)$ and to have these 3 symptoms simultaneously $(57.5 \%$ vs. $18.9 \%, \mathrm{p}<0.001)$. Among children who vomited, frequency of vomiting was higher in $\mathrm{R}+$ than in $\mathrm{R}-(4.3 \mathrm{vs}$. 3.0 per day, $\mathrm{p}<0.001)$. $\mathrm{R}+$ were also more likely to visit an emergency room $(25.5 \%$ vs. $13.0 \%, p=0.004)$, to be hospitalized $(12.0 \%$ vs. $3.0 \%, p<0.001)$ and to require IV-rehydration $(13.0 \%$ vs. $3.0 \%, \mathrm{p}<0.001)$.

CONCLUSION: This study shows that the severity of RGE is clinically more important than other GE. Multiple symptoms increase the risk of rehydration and frequent vomiting makes oral rehydration difficult to perform by parents at home. This may explain why a significant fraction of $\mathrm{R}+$ cases went on to receive additional medical care.

\section{POSTER PRESENTATIONS Immunization Communication Campaign}

\section{P107}

\section{QUALITY IN PRACTICE MADE EASY}

M Watson, Department of Health (DoH) South Australia; L Molchanoff, DoH South Australia; T Harris, DoH South Australia; S Lewis, DoH South Australia; C McInnes, DoH South Australia; M Macaites, DoH South Australia; A Newbound, Adelaide Northern Division of General Practice

INTRODUCTION: The costs associated with the provistion of the National Immunisation Program in Australia, has undergone explosive growth in the past 4 years. The success of the prograpin is defermined by the coverage rates produced by the Austratian fhitdl ood Immunisation Register (ACIR). Those responsible for in im programs have a duty to ensure there /rentorable indicators that can determine the quality and safety aspect be program.

The team of professional the South Australian Immunisation Coordination Unit (8AU hane developed and evaluated a number of resource tools that assist proyiders in achieving the recommended standards. 1. Best practice delivery in a school setting.

Using a collaßpratile approach to ensure consistency in service provision of adolescent vaccines in a school setting, SAICU developed a resource 
tool to support providers and schools to streamline service delivery. The framework comprises of a series of model documents aimed at assisting immunisation providers to meet their professional responsibilities.

2. Immunisation the basics - Education for providers

In Australia, $70 \%$ of childhood immunisations are delivered through General Practice surgeries. Ongoing, accessible education is necessary to ensure service providers are kept informed and updated of all necessary information relating to the program such as legislation, cold chain management, catch-up schedules and vaccine safety. 'Immunisation The Basics' is an adaptable educational tool that allows ongoing education for all those involved in immunisation.

3. Catch up calculator for parents and providers

The calculator is an online computer based system that uses the National Immunisation Program Schedule Australia (NIPSA) to calculate an individual child's immunisation schedule. The calculation is based on their date of birth, immunisation history and the schedule according to the state/territory they live in. This is an easy to use, accurate software program designed to make immunisation catch-up calculation simple.

4. Consent Resource Tool

This waiting room resource tool was developed to assist immunisation providers deliver the necessary information to enable parents and others to reach informed consent for immunisations prior to the consultation thereby reducing time.

\section{P108}

\section{"IF YOU TOKE GET POKED": A SOCIAL MARKETING CAMPAIGN IN RESPONSE TO AN OUTBREAK OF MENINGOCOCCAL C DISEASE IN MEN WHO HAVE SEX WITH MEN IN BC}

E Brodkin, University of British Columbia; M Gilbert, Vancouver Island Health Authority, Victoria, British Columbia; R Gustafson, Vancouver Coastal Health, Vancouver, British Columbia; P Daly, Vancouver Coastal Health, Vancouver, British Columbia

INTRODUCTION AND OBJECTIVES: In December 2004, an outbreak of meningococcal $\mathrm{C}$ infection was identified in men who have sex with men (MSM) in BC, leading to the initiation of an immunization program in this population. All six cases had connections to the Vancouver area, and joint smoking/sharing was identified as a potential risk factor. Three additional cases led to the development of a social marketing campaign in May 2005, with the goals of increasing knowledge of the outbreak and improving vaccine uptake among MSM.

AUDIENCE: The campaign was targeted at gay men in BC (age range teens to 40 years).

CAMPAIGN MESSAGES AND MATERIALS: The key messages were that the outbreak was not over, that the bacteria is spread through saliva including through sharing joints and to get vaccinated. A graphic artist designed slogans/logos for materials that were adapted to print ads, clubcards, posters and websites.

CHANNELS/ACTIVITIES: The campaign ran from May to September 2005 and included: mail-out of posters to physicians, public health units, MSM-oriented businesses and agencies; print ads in gay and other media; bathroom ads; distribution of clubcards; posting to appropriate websites.

EVALUATION: Evaluation occurred through a self-administered survey distributed over Vancouver Pride weekend, August 2005. Knowledge of the three key messages was high among the 417 participants and $66 \%$ of eligible participants had been immunized. Immunized men were more likely to have accurate information regarding meningococcal disease and the outbreak, to report seeing material from the social marketing campaign, and to have a family doctor; while generally favorable, ratings of the ads were not associated with immunization status.

CONCLUSIONS: The majority of men surveyed had been vaccinated prior to May 2005; however, analysis of the timing of participants' vaccination suggested that the campaign had been effective in motivating additional men to undergo immunization.

\section{P109}

IMMUNIZATION EDUCATION INITIATIVE (IEI):

IMMUNIZATION EDUCATION FOR NURSES BY NURSES

L Thompson, Alberta Children's Hospital; MA Carson,

Immunization Education Initiative; D Koch, Immunization

Education Initiative; D Mitchell, Immunization Education Initiative;

C Pollari, Immunization Education Initiative; B Swart,

Immunization Education Initiative

BACKGROUND: Immunization programs in Canada are delivered by a variety of health care providers: pediatricians, general practioners and nurses. Although physicians have numerous opportunities to access immunization education, programs directed to nurse-immunizers are limited. This is especially true for office-based and rural nurses. Nurses also have different education needs compared to physicians; different issues to be addressed.

In 2003 a group of nurses involved in immunization programs from across the country was brought together by the public health education group from Wyeth Pharmaceuticals. The goal was to create an immunization education program for nurses by nurses. They identified potential Advisory Board members, created a needs-assessment tool and mapped out a timeline for project completion. Wyeth agreed to fund the project via an unrestricted financial grant.

IMPLEMENTATION: Within six months, the Advisory Board wrote a series of four articles related to different aspects of immunization. The articles were published in the Canadian Nurse and were well received. The Board then focused on creating an education curriculum comprising of four modules in PowerPoint format: Immunization Overview, Immunology/Vaccinology, Communication Skills and Administration Techniques. In August 2005, the IEI Speakers Bureau was formed. Forty nurses from across the country were invited to Toronto for education sessions based on the four teaching modules and coaching in presentation skills. The participants then returned to their communities to schedule education sessions for local nursing groups. To date, 91 teaching sessions have been completed.

OUTCOME: The teaching modules have been presented to more than 1300 nurses. The response has been very favorable. The module approach allows sessions to be tailored to the individual nursing group and adapted to the time available for the teaching session. Feedback from the sessions indicates the need for further modules addressing travel immunizations and annual influenza vaccination programs/pandemic response.

\section{P110 \\ PROJET VIP - VACCINATION PAR LES INFIRMIÈRES- INFIRMIERS - PROMOTION: ÉLABORATION D'UNE FORMATION SUR LA PRATIQUE VACCINALE ET ÉVALUATION PRÉLIMINAIRE}

G Petit, Université de Montréal, Québec; $M$ Letellier, Direction de santé publique de Montréal, Québec; G Petit, Université de Montréal, Québec; L Gauvin, Université de Montréal, Québec; L Valiquette, Direction de santé publique de Montréal, Québec; C Guimond, Direction de santé publique de Montreal, Québec; N Boulianne, Institut national de santé publique du Québec, Québec

INTRODUCTION: En 2003, les infirmières vaccinatrices du Québec avaient effectué $50 \%$ de la vaccination de base des enfants de $0-5$ ans. Or, une étude de Dionne et al $(1999,2001)$ montrait que les infirmières avaient des croyances moins favorables à la vaccination que les médecins vaccinateurs. Une enquête de 2004 (Petit et al., 2006) suggère que certaines pratiques vaccinales souhaitables sont peu réalisées par les infirmières. Afin de surmonter ce défi, le groupe de recherche VIP a élaboré un programme de formation pour infirmières afin de favoriser l'adoption de pratiques vaccinales qui assureraient une meilleure couverture vaccinale. Le but de cette présentation est de décrire le processus de développement de la formation et de son devis d'évaluation.

MÉTHODOLOGIE: La séquence de formation a été planifiée par consensus du groupe VIP à partir d'une conception socioconstructiviste de l'apprentissage. Des questionnaires visant à documenter les acquis de la 
formation ont aussi été mis au point par processus itératif de consensus. La formation ainsi que la clarté et la pertinence des instruments d'évaluation (tant qualitatifs que quantitatifs) ont été pré-testés auprès de neuf infirmières vaccinatrices.

RÉSULTATS: La formation de 2 jours consiste essentiellement à mobiliser les infirmières autour d'activités permettant d'intégrer des savoirs et de discuter sur les attitudes, croyances et pratiques à partir de situations vaccinales. L'analyse des données provenant des instruments d'évaluation de la formation ont permis de documenter leur clarté et leur pertinence. En particulier, nous avons pu constater la satisfaction des participantes au programme, enrichir les activités prévues, publiciser différemment le programme et produire un guide pour les futures formatrices.

CONCLUSION: Nous concluons qu'il est possible de développer une formation pertinente à la vaccination et des outils utiles par le biais d'un processus de consensus. Le processus d'évaluation de l'efficacité de l'intervention peut donc être amorcé avec confiance.

\section{P111}

IMMUNIZATION RESOURCES TARGETING PARENTS OF YOUNG CHILDREN: GET ALL OF YOUR CHILD'S SHOTS: GET THEM ON TIME!

A Derban, BC Centre for Disease Control; T Tough, TeleCare Management Inc; L Poirier, Ministry of Health; J Pringle, TeleCare Management Inc; H Way, Interior Health Authority; N Chambers, Vancouver Island Health Authority; A Christensen, Northern Health Authority; T Morgana, Vancouver Coastal Health Authority; K McColgan, First Nations Inuit Health Branch

INTRODUCTION: Immunization Coverage Rates for two-year-old children in British Columbia are below target rate until kindergarten age indicating that parents are late for the eighteen month visit. It was essential to determine reasons for this delay and what strategies are necessary to encourage parental compliance.

METHODS: Qualitative research methodology was employed by way of Focus Group Testing of parents of two-year-old children, followed by field testing of new vaccine pamphlets developed as one strategy. Focus groups consisted of two parent groups: those who immunized on time and those who were delayed. A working group, formed to develop strategies related to the key findings, decided to create new vaccine information pamphlets. Five topics were chosen with three to five key messages per topic. A Health Literacy expert and a Graphic Designer were employed to design the pamphlets.

RESULTS: One key finding was that parents in the delayed group were skeptical of the value and/or necessity of immunization. The key message chosen for all pamphlets is: Get Your Child's Shots. Get Them On Time. The specific topics are: Childhood Vaccines: What's In Them and Why, Why Childhood Vaccines are Safe, The Benefits and Risks of Childhood Vaccines, Your Baby's Immune System and Vaccines, and Tips for A Successful Clinic Day. A pamphlet field testing tool is being developed.

EVALUATION: Field testing will commence September 2006 and results will be available by December 2006. Results will guide final additions or omissions of the pamphlets.

CONCLUSIONS: Creating materials based on research with parents is essential to achieve results and qualitative research methods are effective research tools for this purpose. Use of communication expertise increases the effectiveness of the messages. Information pamphlets accessible to all literacy levels addressing parents' specific concerns is one important strategy. 
Please note that a number of corrections have been made to abstracts from the 7th Canadian Immunization Conference, which were published in the November/December 2006 issue of The Canadian Journal of Infectious Diseases $\mathcal{E}$ Medical Microbiology (Can J Infect Dis Med Microbiol 2006;17[6]:347-393). In a few instances, lead authors were not correctly listed.

The corrected abstracts are listed below:

$\mathrm{O} 2$

IMPACT OF A ONE DAY WORKSHOP ON PUBLIC HEALTH PROFESSIONALS' KNOWLEDGE, ATTITUDES AND BELIEFS ON HPV INFECTION, SCREENING AND VACCINATION

B Duval, Institut National de Santé Publique du Québec; V Gilca, Laval University Hospital Centre, Quebec; C Sauvageau, Quebec Regional Public Health Department; F Lavoie, Laval University Hospital Centre, Quebec; P Goggin, Quebec Institute of Public Health; M Steben, Quebec Institute of Public Health

O11

HEALTH PROFESSIONALS SURVEY: KNOWLEDGE, ATTITUDES, AND PRACTICES ABOUT HPV VACCINES USE AND THEIR POTENTIAL IMPACT ON CERVICAL CANCER SCREENING

B Duval, Institut National de Santé Publique du Québec; S Dobson, British Columbia University; I Gemmill, Kingston, Frontenac and Lennox \& Addington Public Health Unit; S McNeil, Dalhousie University; V Gilca, Laval University Hospital Centre; C Sauvageau, Quebec Regional Public Health Unit; F Lavoie, Laval University Hospital Centre

\section{P12}

IMPACT OF ACELLULAR PERTUSSIS VACCINE FOR CHILDREN AND TEENS IN CANADA: SIMULTANEOUS COHORT EFFECTS IN BRITISH COLUMBIA

DT Samara, BC Centre for Disease Control; DM Skowronski, BC Centre for Disease Control; SA Tweed, BC Centre for Disease Control; C Shaw, BC Centre for Disease Control; G De Serres, Universite Laval; S Halperin, Dalhousie University; A Coombs, Nova Scotia Department of Health; J Isaac-Renton, BC Centre for Disease Control

\section{P14}

PENTA- AND HEXAVALENT DTAP BASED VACCINES APPROVED FOR USE IN CANADA: A SYSTEMATIC LITERATURE REVIEW OF IMMUNOGENICITY AND REACTOGENICITY

V Gilca, Laval University Hospital Centre; B Duval, Institut National de Santé Publique du Québec; SH Deeks, Public Health Agency of Canada; K Laupland, University of Calgary

P16

EFFICACY OF 23-VALENT POLYSACCHARIDE PNEU. MOCOCCAL VACCINE (PPV) AGAINST INVASIVE PNEUMOCOCCAL INFECTION AND PNEUMONIA, ONTARIO

Z Liu, Mount Sinai Hospital; KA Green, Mount Sinai Hospital; M Lovgren, National Centre for Streptococcus; G Tyrrell, National Centre for Streptococcus; A McGeer, Mount Sinai Hospital

\section{P46}

CERVICAL CANCER SCREENING AND EVENTUAL HPV VACCINE ACCEPTABILITY: AN EXPLORATORY SURVEY IN CANADIAN ADULTS

C Sauvageau, Quebec Regional Public Health Department; B Duval, Institut National de Santé Publique du Québec; V Gilca, Laval University Hospital Centre; F Lavoie, Laval University Hospital Centre; M Ouakki, Laval University Hospital Centre

\section{P47}

KEEPING THEM IN: PARTICIPANT RETENTION IN LONG TERM IMMUNOGENICITY - BOOSTER STUDIES

B Duval, Institut National de Santé Publique du Québec; C Couture, Public Health Research Unit, Laval University Hospital Centre; F Lavoie, Public Health Research Unit, Laval University Hospital Centre; S Auger, Public Health Research Unit, Laval University Hospital Centre; V Gilca, Public Health Research Unit, Laval University Hospital Centre

P58

COST ANALYSIS OF PUBLIC HEALTH DELIVERY OF CHILDHOOD IMMUNIZATIONS IN NOVA SCOTIA

C Michaels, Dalhousie University; L Kumaranayake, Dalhousie University; J Scott, Nova Scotia Department of Health Promotion and Protection; S Sarwal, Nova Scotia Department of Health Promotion and Protection; A Coombs, Nova Scotia Department of Health Promotion and Protection; E Holmes, Nova Scotia Department of Health Promotion and Protection; I Sketris, Dalhousie University

P82

AGE-RELATED TRENDS IN THE TIMELINESS AND PREDICTIVENESS OF MEDICAL VISITS, HOSPITALIZATIONS AND DEATHS DUE TO PNEUMONIA AND INFLUENZA

R Sebastian, BC Centre for Disease Control; M Chong, BC Centre for Disease Control; DM Skowronski, BC Centre for Disease Control; J Dhaliwal, BC Centre for Disease Control; J Brownstein, Harvard Medical School, Boston, Massachusetts, USA 


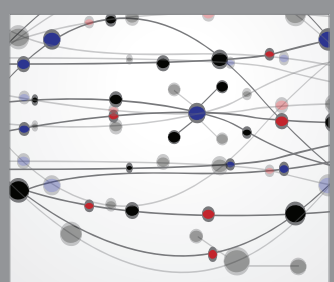

The Scientific World Journal
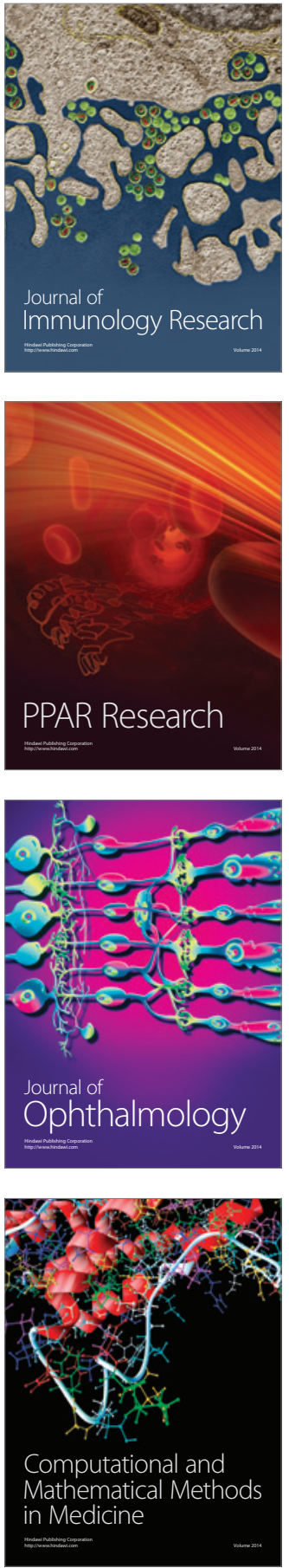

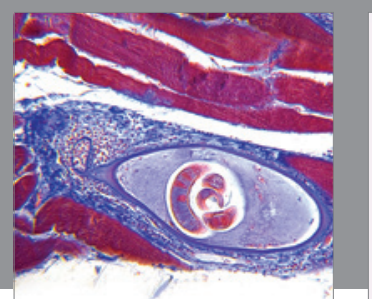

Gastroenterology Research and Practice

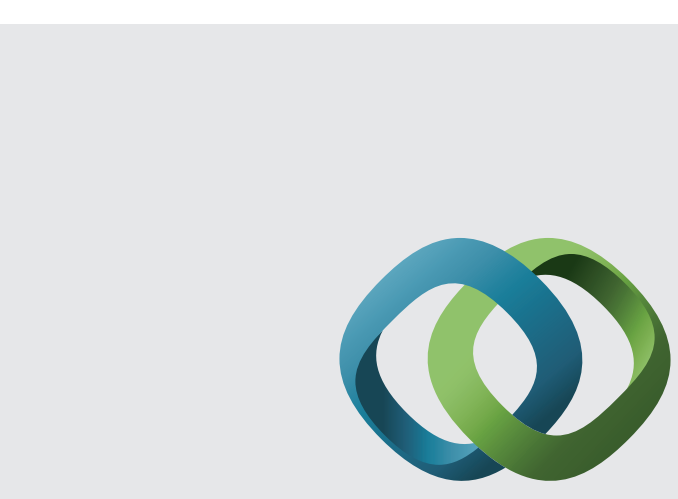

\section{Hindawi}

Submit your manuscripts at

http://www.hindawi.com
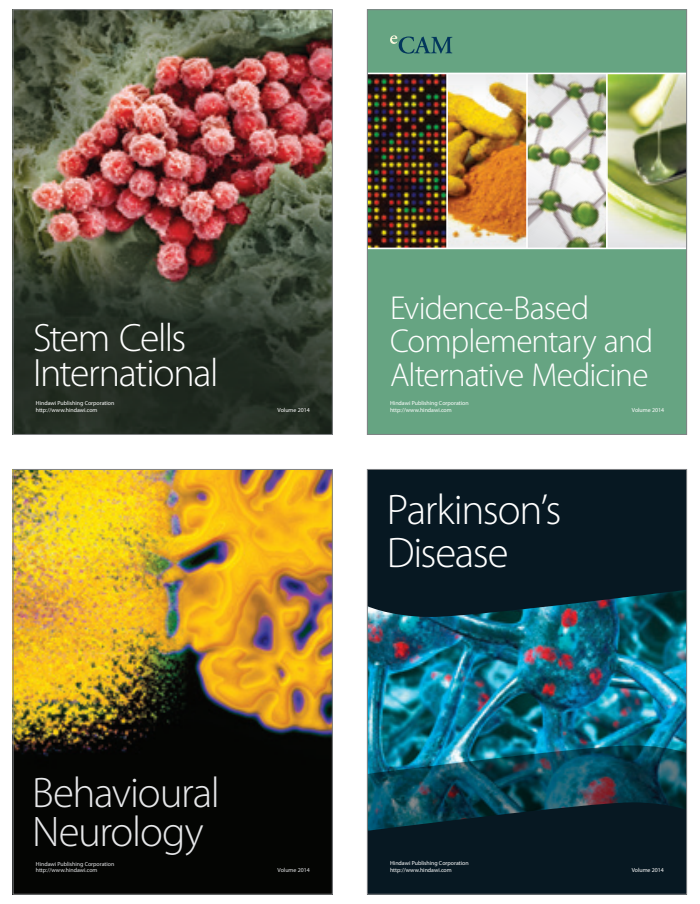
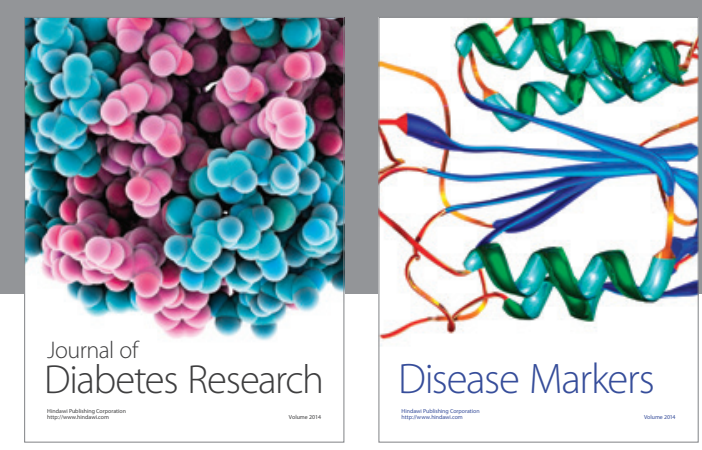

Disease Markers
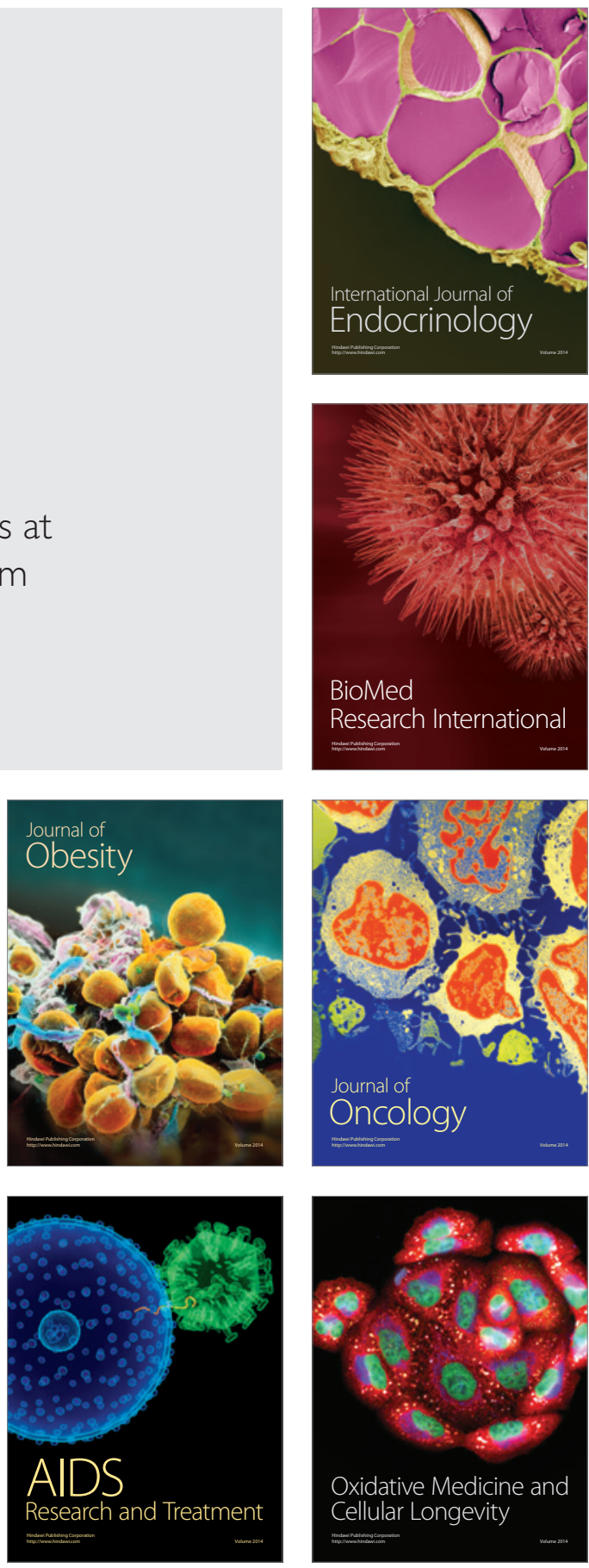